LBL --29573

DE91 004314

September 11, 1990

\title{
Differential Geometry of Groups in String Theory *
}

\author{
Ph.D. Thesis \\ William B. Schmidke, Jr. \\ Theoretical Physics Group \\ Physics Division \\ Lawrence Berkeley Laboratory \\ 1 Cyclotron Road \\ Berkeley, California 94720
}

\begin{abstract}
Techniques from differential geometry and group theory are applied to two topics from string theory. The first topic studied is quantum groups, with the example of $\mathrm{GL}(1 \mid 1)$. The quantum group $\mathrm{GL}_{q}(1 \mid 1)$ is introduced, and an exponential description is derived. The algebra and coproduct are determined using the invariant differential calculus method introduced by Woronowicz and generalized by Wess and Zumino. An invariant calculus is also introduced on the quantum superplane, and a representation of the algebra of $\mathrm{GL}_{q}(1 \mid 1)$ in terms of the super-plane coordinates is constructed.

The second topic follows the approach to string theory introduced by Bowick and Rajeev. Here the ghost contribution to the anomaly of the energy-momentum tensor is calculated as the Ricci curvature of the Kähler quotient space $\operatorname{Diff}\left(S^{1}\right) / S^{1}$. We discuss general Kähler quotient spaces and derive an expression for their Ricci curvatures. Application is made to the string and superstring diffeomorphism groups, considering all possible choices of subgroup. The formalism is extended to associated holomorphic vector bundles, where the Ricci curvature corresponds to the anomaly for different ghost sea levels.
\end{abstract}

*This work was supported by the Director, Office of Energy Research, Office of High Energy and Nuclear Physics, Division of High Energy Physics of the U.S. Department of Energy under Contract DE-AC03-76SF00038. 


\section{Acknowledgements}

I would like to thank my adviser, Professor Bruno Zumino, for giving me the opportunity to pursue this work and for his endless patierice with me. It has been a privelege to learn his elegant approach to physics.

I also thank Professor Marty Halpern whose excellent teaching inspired me to pursue this line of research. His support and encouragement have been a constant help.

I wish to thank the many other members of the theory group for advice and companionship throughout the course of this work. Special thanks to Betty Moura and Luanne Neumann for providing a warm and hospitable environment.

I also thank the many experimental physicists with whom I have collaborated over the course of my studies. Working with them has provided a well rounded physics education not common in the field these days.

My life in Berkeley has been enormously enriched by the wide variety of friends I have had over the years. Most of them fall into one of the following categories: the Sequoia residents; the Deadheads; the Texans; the Northside (Oakland) crowd; the Tuesday night softball group; and the Degenerate Vacua. I thank them all for their companionship which has kept me sane.

I dedicate this work to my parents, Helen and Bill Sr., whose love and support has made everything possible. 


\section{Contents}

1 Introduction 1

2 The Quantum Group $\mathrm{GL}_{\mathrm{q}}(1 \mid 1)$

2.1 R-matrix Quantization of Groups . . . . . . . . . . . . . 4

2.2 R-matrix and q-relations for $\mathrm{GL}_{\mathrm{q}}(1 \mid 1) \ldots \ldots \ldots$

2.3 The Exponential Description . . . . . . . . . . . . . 9

3 The Quantum Algebra GL $(1 \mid 1) \quad 13$

3.1 Exterior Derivative and Cartan-Maurer Forms ........... 13

3.2 Additional q-relations . . . . . . . . . . . . . . 15

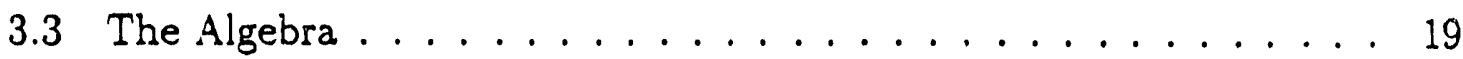

3.4 The Coproduct ...................... 21

3.5 Method of Faddeev, Reshetikhin, and Takhtajan . . . . . . . . . . 24

4 The Quantum Super-Plane $\quad 28$

4.1 Coordinate and Differential q-relations ............. 28

4.2 Super-Plane Exterior Derivative ................. . 31

4.3 Additional q-relations . . . . . . . . . . . . . 33

4.4 Derivative q-relations .................... 34 
4.5 Representation of Derivatives ............... 36

4.6 Representation of the Algebri. . . . . . . . . . . . . . 40

5 Quotient Space Geometry 43

5.1 Quotient Space $G / H \ldots \ldots . \ldots . \ldots 43$

5.2 Riemannian Geometry of $G / H \ldots \ldots$. . . . . . . . . . 45

5.3 Almost Complex Structure on $G / H \ldots \ldots$. . . . . . . . 46

5.4 Complex Structure on $G / H \ldots \ldots \ldots$. . . . . . . . 47

5.5 Kählerian Geometry of $G / H \ldots \ldots$. . . . . . . . . . . 48

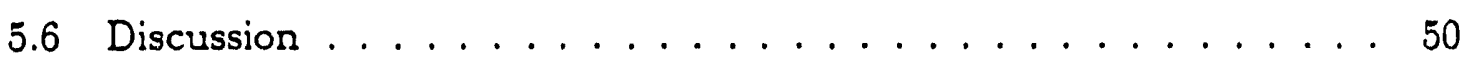

6 String Diffeomorphism Groups 52

6.1 Quotient Spaces for the String . . . . . . . . . . . . . 52

6.2 Quotient Spaces for the Superstring . . . . . . . . . . . 55

6.3 Graded Algebras ..................... 56

7 Associated Vector Bundles $\quad 58$

7.1 Holomorphic Vector Bundles . . . . . . . . . . . . . . 58

7.2 Implications for String Theory . . . . . . . . . . . . . . 61

A Super-Tensor Index Conventions 63

A.1 Super-Functions and Derivatives .............. 63

A.2 Super-Vectors ....................... 64

A.3 Super-Tensors ....................... . . 67 


\section{Chapter 1}

\section{Introduction}

In recent years string theory has been dominated by the application of higher mathematics to theoretical physics. New mathematical structures unfamiliar to physicists have arisen in many studies. Mathematics well known to physicists has also been applied in new and powerful approaches. In this work we focus on the application of two branches of mathematics already common in theoretical physics: differential geometry and group theory. We will use techniques from both of these fields to study two different topics arising in string theory.

The first topic we discuss concerns quantum groups. Quantum groups arise naturally in several aspects of physics and mathematics, namely in the context of integrable models, quantum inverse scattering methods, the KnizhnikZamolodchikov equation, rational conformal field theory, as well as in the theory of Hopf algebras, solution of Yang-Baxter equations and theory of braids [1, 2, 3, 4]. For our purposes quantum groups can be thought of as one-parameter deformations of classical groups. We will concentrate on matrix groups. In this case the elements of a quantum matrix do not behave like ordinary c-numbers, but obey modined commutation rules imposed by the quantization procedure. Given these rules, the problem is to derive the algebra of the generators of the quantum group. For matrix groups one may also consider the vector space acted on by the matrices. The components of these vectors also obey modified commutation relations. This approach to quantum groups has been developed by Manin [5]. 
In this work we quantize the group GL(1|1). This is the group of nonsingular matrices acting on the superplane, a vector space with one commuting and one Grassmann coordinate. We quantize using the R-matrix procedure outlined in [6, 7]. Using the relations on matrix elements imposed by quantization, we determine the algebra of the generators. We follow the invariant differential calculus method introduced by Woronowicz [8] and generalized by Wess and Zumino [9]. This calculus is based on an exterior derivative which is nilpotent and obeys the Leibniz rule. The method also yields the coproduct, which describes how the generators act on a tensor product of spaces. Finally we construct an invariant differential calculus on the superplane. We also construct a representation of the quantum group generators based on the superplane coordinates.

The second main topic of this work concerns an approach to string theory initiated by Bowick and Rajeev [10]. They formulated open string theory in terms of holomorphic line bundles over the manifold $\operatorname{Diff}\left(\mathrm{S}^{1}\right) / \mathrm{S}^{1}$. This was motivated by the fact that the quantization procedure is not invariant under reparameterizations of the string coordinate. The open string may be described as a loop in phase space, and reparameterizations form the group of diffeomorphisms of the circle Diff $\left(\mathrm{S}^{1}\right)$. The action of $\operatorname{Diff}\left(\mathrm{S}^{1}\right)$ mixes creation and annihilation operators, changing the definition of the vacuum state. The subgroup of $\operatorname{Diff}\left(\mathrm{S}^{1}\right)$ consisting of the rigid rotations of the circle $\left(\cong U(1) \cong \mathrm{S}^{1}\right)$ does not mix the operators. Thus, the inequivalent vacua may be identified with the quotient space $\mathcal{M} \equiv \operatorname{Diff}\left(\mathrm{S}^{1}\right) / \mathrm{S}^{1}$. $\mathcal{M}$ is an infinite dimensional Kähler manifold. Each fiber of the string vacuum bundle over $\mathcal{M}$ consists of the open string Fock space with the usual inner product. The curvature of this bundle is the anomaly in the Virasoro algebra. Geometrically, non-vanishing curvature means that a covariantly constant section of the bundle does not exist; one cannot define a reparameterization invariant vacuum. In reference [10] ghosts and their associated vacuum bundle are included, cancelling the anomaly in the critical dimension. The authors also noted that the curvature of the ghost vacuum bundle is equal to the curvature of the canonical line bundle over $\mathcal{M}$, which for a Kähler manifold is given by the Ricci form. This motivates our interest in the geometry of $\operatorname{Diff}\left(S^{1}\right) / S^{1}$ and related manifolds. Subsequent work has further clarified the Riemannian geometry of $\operatorname{Diff}\left(S^{1}\right) / S^{1}[11]$ and extended the 
calculation to the supersymmetric case super-Diff($\left(\mathrm{S}^{1}\right) / \mathrm{S}^{1}[12,13,14,15,16]$.

In this work we discuss the calculation of the curvature of the canonical line bundle of a quotient space and extend the formalism to include other levels of the ghost vacuum. The discussion uses the language of differential geometry familiar to physicists. We begin by reviewing the construction of a quotient space $\mathcal{M}=G / H$ for a group $G$ with subgroup $H$. The Riemannian geometry of such a space for $G$-invariant metrics is discussed. Next the conditions for $\mathcal{M}$ to be a complex manifold are outlined. If $M$ admits a Kähler metric the associated Ricci curvature has a simple form independent of the metric. These results are then applied to the groups relevant to the string and superstring, which are special cases of graded algebras. Here it is seen that the use of the Kähler metric regulates otherwise divergent quantities. Finally, the calculation of the Ricci curvature is extended from the tangent bundle of Riemann and Kähler geometry to more general vecto: bundles based on these spaces. This result corresponds to different filling levels of the ghost sea. 


\section{Chapter 2}

\section{The Quantum Group $\mathrm{GL}_{\mathrm{q}}(1 \mid 1)$}

In this chapter we discuss the quantum group $\mathrm{GL}_{\mathbf{q}}(1 \mid 1)$. The classical matrix group is quantized using the R-matrix procedure, following closely the discussion in [7]. This determines the bilinear quantum relations on the matrix elements. Covariance of these relations is demonstrated. The quantum determinant $:=1$ so introduces. Finally, an exponential description of the quantum group is developed.

\subsection{R-matrix Cuantization of Groups}

The method of group quantization most commonly encountered for matrix groups, involves the $\mathrm{R}$-matrix. The $\mathrm{R}$-matrix is in the tensor product of two matrix spaces. It describes how to reverse the ordering of the same matrix in two different tensor spaces. Explicitly, if the same matrix $A$ is written as $A_{1}=A \otimes 1$ when in the first space and $A_{2}=1 \otimes A$ in the second, then the R-matrix equation is

$$
R A_{1} A_{2}=A_{2} A_{1} R
$$

This equation defines bilinear relations on the elements of $A$. We will refer to such relations defined by quantization as q-relations.

If one considers the triple tensor product of matrices, consistency determines a constraint in the $R$-matrix. Let $R_{(12)}$ act on the 1 and 2 matrix spaces, $R_{(13)}$ on the 1 and 3 spaces, and $R_{(23)}$ on the 2 and 3 spaces. The R-matrix commutes 
with matrices not in the space it acts on. Then using the above $\mathrm{R}$-matrix equation repeatedly to switch matrices shows

$$
\begin{aligned}
R_{(23)} R_{(13)} R_{(12)} A_{1} A_{2} A_{3} & =R_{(23)} R_{(13)} A_{2} A_{1} A_{3} R_{(12)} \\
& =R_{(23)} A_{2} A_{3} A_{1} R_{(13)} R_{(12)} \\
& =A_{3} A_{2} A_{1} R_{(23)} R_{(13)} R_{(12)}
\end{aligned}
$$

and we see that $R_{(23)} R_{(13)} R_{(12)}$ reverse the order of $A_{1} A_{2} A_{3}$ to $A_{3} A_{2} A_{1}$. However, the order in which we swapped matrices can be changed. The other way is

$$
\begin{aligned}
R_{(12)} R_{(13)} R_{(23)} A_{1} A_{2} A_{3} & =R_{(12)} R_{(13)} A_{1} A_{3} A_{2} R_{(23)} \\
& =R_{(12)} A_{3} A_{1} A_{2} R_{(13)} R_{(23)} \\
& =A_{3} A_{2} A_{1} R_{(12)} R_{(13)} R_{(23)} .
\end{aligned}
$$

Here we see that $R_{(12)} R_{(13)} R_{(23)}$ switches the ordering as before. Thus these two triple products of $R$-matrices are equal:

$$
R_{(12)} R_{(13)} R_{(23)}=R_{(23)} R_{(13)} R_{(12)}
$$

This is the Yang-Baxter equation. No further constraints arise from considering higher tensor products.

\subsection{R-matrix and q-relations for $\mathrm{GL}_{\mathrm{q}}(1 \mid 1)$}

The classical group GL(1|1) is the group of $2 \times 2$ non-sigular super-matrices which act on a 2-dimensional vector space with one commuting and one anticommuting coordinate. Such a matrix $A \in \mathrm{GL}(1 \mid 1)$ can be written

$$
A=\left(\begin{array}{ll}
a & \beta \\
\gamma & d
\end{array}\right)
$$

where the matrix elements $a$ and $d$ are commuting and $\beta$ and $\gamma$ are anticommuting. The (anti-)commutation rules will be modified by quantization.

The $\mathrm{R}$-matrix for $\mathrm{GL}_{\mathrm{q}}(1 \mid 1)$ is in the tensor product of $2 \times 2$ super-matrices, so it is a $4 \times 4$ matrix. As discussed in the appendix, it is convenient to choose 
an index ordering convention which allows simple matrix multiplcation with no additional grading signs. We choose the indices ordered as $R^{i_{1} i_{2}}{ }_{j_{2} j_{1}}$, where the subscripts on the indices indicate which vector space in the tensor product the index belongs to. The the $\mathrm{R}$-matrix for $\mathrm{GL}_{\mathrm{q}}(1 \mid 1)$ is

$$
R=\left(\begin{array}{cccc}
q & 0 & 0 & 0 \\
0 & 1 & 0 & 0 \\
0 & \lambda & 1 & 0 \\
0 & 0 & 0 & q^{-1}
\end{array}\right)
$$

where $\lambda=q-q^{-1}$. This R-matrix has been used extensively in the work of Lee and Couture [17].

It can be verified that this $R$-matrix satisfies the Yang-Baxter equation (2.2). For this the R-matrix is written as an $8^{*} \times 8$ matrix in the tensor product of $32 \times 2$ super-matrices. Again it is convenient to choose index ordering for simple matrix multiplication. In terms of the above index ordering for $R$, we have

$$
\begin{aligned}
& R_{(12)}^{i_{1} i_{2} i_{3}}{ }_{j 3 j_{2} j_{1}}=R^{i_{1} i_{2}}{ }_{j_{2} j_{1}} \delta^{i_{3}}{ }_{j_{3}} \\
& R_{(13){ }_{j 3} i_{2} j_{1} j_{1}}^{i_{13}}=(-1)^{i_{2}\left(i_{3}+j_{3}\right)} R^{i_{1} i_{3}}{ }_{j_{3} j_{1}} \delta^{i_{2}}{ }_{j_{2}} \\
& R_{(23){ }^{2} i_{3} i_{2} j_{3} j_{1}}=(-1)^{i_{1}\left(i_{2}+i_{3}+j_{2}+j_{3}\right)} R^{i_{2} i_{3}}{ }_{j_{3} j_{2}} \delta^{i_{1}}{ }_{j_{1}} .
\end{aligned}
$$

Here the $Z_{2}$ grading signs are $(-1)^{i}=+1$ for index $i$ corresponding to the first row or column of a $2 \times 2$ matrix space, and $(-1)^{i}=-1$ for $i$ correspondin $\tilde{E}$ to the second row or column.

Having verified that the $\mathrm{R}$-matrix satisfies the Yang-Baxter equation. we can now use it in (2.1) to determine q-relations on the elements of a matrix $A$ as in (2.3). In writing the $4 \times 4$ matrices corresponding to $A_{1} A_{2}$ and $A_{2} A_{1}$ we once again choose index ordering for simple matrix multiplication. Then the grading sigus are given by

$$
\begin{aligned}
& \left(A_{1}\right)^{i_{1} i_{2}}{ }_{j_{2} j_{1}}=(A \otimes 1)^{i_{1} i_{2}}{ }_{j_{2} j_{1}}=(-1)^{j_{1}\left(i_{2}+j_{2}\right)} A^{i_{1}}{ }_{j_{1}} \delta^{i_{2}}{ }_{j_{2}} \\
& \left(A_{2}\right)^{i_{1} i_{2}}{ }_{j_{2} j_{1}}=(1 \otimes A)^{i_{1} i_{2}}{ }_{j_{2} j_{1}}=(-1)^{i_{1}\left(i_{2}+j_{2}\right)} A^{i_{2}}{ }_{j_{2}} \delta^{i_{1}}{ }_{j_{1}}
\end{aligned}
$$


so the $4 \times 4$ matrices are

$$
A_{1}=\left(\begin{array}{llll}
a & 0 & \beta & 0 \\
0 & a & 0 & \beta \\
\gamma & 0 & d & 0 \\
0 & \gamma & 0 & d
\end{array}\right) \quad A_{2}=\left(\begin{array}{cccc}
a & \beta & 0 & 0 \\
\gamma & d & 0 & 0 \\
0 & 0 & a & -\beta \\
0 & 0 & -\gamma & d
\end{array}\right) .
$$

Using these matrices and the R-matrix (2.4) in equation (2.1) gives bilinear :relations for the elements of $A$. The independent q-relations are

$$
\begin{array}{ll}
a \beta=q \beta a & \beta^{2}=0 \\
a \gamma=q \gamma a & \gamma^{2}=0 \\
d \beta=q \beta d & \beta \gamma=-\gamma \beta \\
d \gamma=q \gamma d & a d-d a=-\lambda \beta \gamma
\end{array}
$$

where $\lambda=q-q^{-1}$. Note that the first three relations in the right column are the sarne as in the classical $(q=1)$ case. The other relations are modified by quantization.

The determinant of the quantum matrix $A$ may be defined in the same way as for a classical matrix. Begin by decomposing the matrix into a product of a lower triangular matrix, a diagonal matrix, and an upper triangular matrix as follows:

$$
A=\left(\begin{array}{ll}
a & \beta \\
\gamma & d
\end{array}\right)=\left(\begin{array}{cc}
1 & 0 \\
\gamma a^{-1} & 1
\end{array}\right)\left(\begin{array}{cc}
a & 0 \\
0 & d-\gamma a^{-1} \beta
\end{array}\right)\left(\begin{array}{cc}
1 & a^{-1} \beta \\
0 & 1
\end{array}\right) .
$$

The determinant of $A$ is the product of the determinants of each of these three matrices. The determinants of the triangular matrices are one. The determinant of a diagonal super-matrix is the product of the diagonal elements in bosonic rows divided by the diagonal elements in fermionic rows. This is a result of the fact that the super-trace of a super-matrix is the sum of the bosonic diagonal elements minus the fermionic elements. The determinant of $A$ is then the exponential of the super-trace of $\ln A$. (Later we will demonstrate that this is also true for these quantum inatrices.)

Using the above decomposition and rule for determinant of diagonal supermatrix gives

$$
\operatorname{det}(A)=a\left(d-\gamma a^{-1} \beta\right)^{-1} .
$$


The term in parentheses is easy to invert since the Grassmann elements are nilpotent. Then using the q-relations to rearrange the group parameters (note that no net factors of $q$ appear) we find

$$
\begin{aligned}
\operatorname{det}(A) & =a d^{-1}-\beta d^{-1} \gamma d^{-1} \\
& =d^{-1} a-d^{-1} \beta d^{-1} \gamma .
\end{aligned}
$$

This can be shown to obey the usual properties of the determinant, for example $\operatorname{det}(A) \operatorname{det}(B)=\operatorname{det}(A B)$. More importantly, the determinant is central, i.e. it commutes with all of the matrix elements:

$$
\operatorname{det}(A) A=A \operatorname{det}(A) .
$$

Later we will also consider the group $\mathrm{SL}_{\mathrm{q}}(1 \mid 1)$, the group of $2 \times 2$ super-matrices with determinant one.

One interesting property of the quantum groups is covariance of the qrelations (2.5). This means that the product of two different quantum matrices with elements satisfying the q-relations (2.5) produces a new quantum matrix with elements satisfying the same relations. Explicitly, consider two matrices $A$ and $A^{\prime}$ with elements

$$
A=\left(\begin{array}{ll}
a & \beta \\
\gamma & d
\end{array}\right) \quad A^{\prime}=\left(\begin{array}{ll}
a^{\prime} & \beta^{\prime} \\
\gamma^{\prime} & d^{\prime}
\end{array}\right) .
$$

The elements of each matrix satisfy the q-relations (2.5), e.g. $a \beta=q \beta a, a^{\prime} \beta^{\prime}=$ $q \beta^{\prime} a^{\prime}$, etc. The elemenmts of $A$ (anti-)commute with those of $A^{\prime}$. The product matrix $A^{\prime \prime}$ is defined by

$$
A^{\prime \prime}=A A^{\prime}=\left(\begin{array}{ll}
a^{\prime \prime} & \beta^{\prime \prime} \\
\gamma^{\prime \prime} & d^{\prime \prime}
\end{array}\right) .
$$

It is straightforward to check that the elements of $A^{\prime \prime}$ given by

$$
\begin{array}{ll}
a^{\prime \prime}=a a^{\prime}+\beta \gamma^{\prime} . & \beta^{\prime \prime}=a \beta^{\prime}+\beta d^{\prime} \\
\gamma^{\prime \prime}=\gamma a^{\prime}+d \gamma^{\prime} & d^{\prime \prime}=\gamma \beta^{\prime}+d d^{\prime}
\end{array}
$$

also satisfy the q-relations, e.g. $a^{\prime \prime} \beta^{\prime \prime}=q \beta^{\prime \prime} a^{\prime \prime}$. It is in this sense that one says that the quantum group $\mathrm{GL}_{\mathbf{q}}(1 \mid 1)$ is closed under multiplication. The product of two different elements of $\mathrm{GL}_{\mathbf{q}}(1 \mid 1)$ produces another element of $\mathrm{GL}_{\mathbf{q}}(1 \mid 1)$. 


\subsection{The Exponential Description}

Another interesting property of the quantum group $\mathrm{GL}_{\mathbf{q}}(1 \mid 1)$ is found when considering powers of an element of the group. Consider the matrix $A^{2}$ :

$$
A^{2}=A A=\left(\begin{array}{ll}
a_{2} & \beta_{2} \\
\gamma_{2} & d_{2}
\end{array}\right)
$$

with elements

$$
\begin{array}{ll}
a_{2}=a^{2}+\beta \gamma & \beta_{2}=a \beta+\beta d \\
\gamma_{2}=\gamma a+d \gamma & d_{2}=\gamma \beta+d^{2} .
\end{array}
$$

It is straightforward to verify that the elements of $A^{2}$ satisfy the q-relations (2.5) with $q$ replaced by $q^{2}$, or explicitly

$$
\begin{array}{ll}
a_{2} \beta_{2}=q^{2} \beta_{2} a_{2} & \beta_{2}^{2}=0 \\
a_{2} \gamma_{2}=q^{2} \gamma_{2} a_{2} & \gamma_{2}^{2}=0 \\
d_{2} \beta_{2}=q^{2} \beta_{2} d_{2} & \beta_{2} \gamma_{2}=-\gamma_{2} \beta_{2} \\
d_{2} \gamma_{2}=q^{2} \gamma_{2} d_{2} & a_{2} d_{2}-d_{2} a_{2}=-\left(q^{2}-q^{-2}\right) \beta_{2} \gamma_{2} .
\end{array}
$$

This means that if $A \in \mathrm{GL}_{\mathbf{q}}(1 \mid 1)$, then $A^{2} \in \mathrm{GL}_{\mathbf{q}^{2}}(1 \mid 1)$. This suggests that similar relations will hold for any power of $A$. This has been verified through detailed calculations as discussed in [18]. Here we will demonstrate this through a differen; approach.

Because the group $\mathrm{GL}_{q}(1 \mid 1)$ is relatively simple, it is possible to explicitly check many of its properties. Here we will consider an exponential description of group elements. Write an element $A$ of $\mathrm{GL}_{\mathbf{q}}(1 \mid 1)$ as the exponential of another matrix $M$ times a parameter $h$ defined by

$$
A=e^{h M} \quad q=e^{h} .
$$

The elements of $A$ and $M$ are

$$
A=\left(\begin{array}{ll}
a & \beta \\
\gamma & d
\end{array}\right) \quad M=\left(\begin{array}{ll}
r & \sigma \\
\tau & u
\end{array}\right) .
$$

Like the elements of $A$, the elements of $M$ will not obey ordinary graded commutation rules. The corret relations can be determined by writing the elements of 
$A$ to order $h^{2}$ and plugging into the q-relations (2.5). One finds that the elements of $M$ must obey

$$
\begin{array}{ll}
r \sigma-\sigma r=\sigma & r u=u r \\
u \sigma-\sigma u=\sigma & \sigma \tau=-\tau \sigma \\
r \tau-\tau r=\tau & \sigma^{2}=0 \\
u \tau-\tau u=\tau & \tau^{2}=0 .
\end{array}
$$

The relations in the right column are the usual graded commutation relations; the left column is modified. Note that $q$ (or $h$ ) does not appear in these relations. Also note that the super-trace of $M,(r-u)$, commutes with all of the elements.

Using these relations, an explicit form of the exponential can be written. This is easy to do because of the nilpotency of $\sigma$ and $\tau$ in (2.8). Write $M$ as a sum of two matrices as follows:

$$
M=M_{0}+M_{1} \quad M_{0}=\left(\begin{array}{cc}
r & 0 \\
0 & u
\end{array}\right) \quad M_{1}=\left(\begin{array}{cc}
0 & \sigma \\
\tau & 0
\end{array}\right) .
$$

Because of nilpotency, $M_{1}^{n>2}$ varishes. Then any power of $M$ greater than or equal to two may be written

$$
M^{n \geq 2}=M_{0}^{n}+\sum_{m=0}^{n-1} M_{0}^{m} M_{1} M_{0}^{n-m-1}+\sum_{m=0}^{n-2} \sum_{l=0}^{n-m-2} M_{0}^{m} M_{1} M_{0}^{l} M_{1} M_{0}^{n-m-l-2} .
$$

Using the relations (2.8), the terms in the summations can be written in a simple form. The needed expressions are

$$
\begin{aligned}
& M_{0}^{n}=\left(\begin{array}{cc}
r^{n} & 0 \\
0 & u^{n}
\end{array}\right) \\
& M_{0}^{m} M_{1} M_{0}^{l}=\left(\begin{array}{cc}
0 & \sigma(r+1)^{m} u^{l} \\
\tau(u+1)^{m} r^{l} & 0
\end{array}\right) \\
& M_{0}^{m} M_{1} M_{0}^{l} M_{1} M_{0}^{k}=\left(\begin{array}{cc}
\sigma \tau(r+2)^{m}(u+1)^{l} r^{k} & 0 \\
0 & -\sigma \tau(u+2)^{m}(r+1)^{l} u^{k}
\end{array}\right) .
\end{aligned}
$$

Using these expressions in the expansion of $M^{n}$, the summations can be performed leaving

$$
M M^{n}=\left(\begin{array}{ll}
r_{n} & \sigma_{n} \\
\tau_{n} & u_{n}
\end{array}\right)
$$


where

$$
\begin{aligned}
& r_{n}=r^{n}+\sigma \tau\left(\frac{(r+2)^{n}}{2(1+r-u)}+\frac{r^{n}}{2(1-r+u)}-\frac{(u+1)^{n}}{1-(r-u)^{2}}\right) \\
& \sigma_{n}=\sigma \frac{(r+1)^{n}-u^{n}}{1+r-u} \\
& \tau_{n}=\tau \frac{(u+1)^{n}-r^{n}}{1-r+u} \\
& u_{n}=u^{n}-\sigma \tau\left(\frac{(u+2)^{n}}{2(1-r+u)}+\frac{u^{n}}{2(1+r-u)}-\frac{(r+1)^{n}}{1-(r-u)^{2}}\right) .
\end{aligned}
$$

Finally, the exponential sum

$$
e^{h M}=\sum_{n=0}^{\infty} \frac{h^{n}}{n !} M^{n}
$$

may be performed, yielding the elements of the matrix $A$. One finds

$$
\begin{aligned}
& a=e^{h r}+\frac{\sigma \tau}{1-(r-u)^{2}}\left(\frac{1}{2}(1-r+u) e^{h(r+2)}+\frac{1}{2}(1+r-u) e^{h r}-e^{h(u+1)}\right) \\
& \beta=\frac{\sigma}{1+r-u}\left(e^{h(r+1)}-e^{h u}\right) \\
& \gamma=\frac{\tau}{1-r+u}\left(e^{h(u+1)}-e^{h r}\right) \\
& d=e^{h u}-\frac{\sigma \tau}{1-(r-u)^{2}}\left(\frac{1}{2}(1+r-u) e^{h(u+2)}+\frac{1}{2}(1-r+u) e^{h u}-e^{h(r+1)}\right) .
\end{aligned}
$$

Note that these expressions have been written with no explicit appearance of $q$. All dependence on $q$ is carried by $h$.

It is straightforward to verify, using (2.8), that the above expressions for the elements of $A$ satisfy the q-relations (2.5), with $q=e^{h}$. Now consider $A^{p}$, where $p$ is any $c$-number. From (2.7) we see that this may be written

$$
A^{p}=\left(e^{h M}\right)^{p}=e^{p h M}
$$

Then the elements of $A^{p}$ :

$$
A^{p}=\left(\begin{array}{ll}
a_{p} & \beta_{p} \\
\gamma_{p} & d_{p}
\end{array}\right)
$$

are obtained from (2.9) with the substitution $h \rightarrow p h$, with the elements of $M$ $\left(r, \sigma, \tau\right.$, and $u$ ) still satisfying (2.8). The q-relations for the elements of $A^{p}$ are 
satisfied with the same substitution, with $q \rightarrow q^{p}$. Explicitly,

$$
\begin{array}{lll}
a_{p} \beta_{p}=q^{p} \beta_{p} a_{p} & d_{p} \beta_{p}=q^{p} \beta_{p} d_{p} & \beta_{p} \gamma_{p}=-\gamma_{p} \beta_{p} \\
a_{p} \gamma_{p}=q^{p} \gamma_{p} a_{p} & d_{p} \gamma_{p}=q^{p} \gamma_{p} d_{p} & a_{p} d_{p}-d_{p} a_{p}=-\left(q^{p}-q^{-p}\right) \beta_{p} \gamma_{p}
\end{array}
$$

and $\beta_{p}$ and $\gamma_{p}$ are nilpotent as usual. This verifies the property of $\mathrm{GL}_{q}(1 \mid 1)$ suggested by $A^{2}$ mentioned earlier. If $A \in \mathrm{GL}_{\mathrm{q}}(1 \mid 1)$, then $A^{\mathrm{p}} \in \mathrm{GL}_{\mathrm{q}}(1 \mid 1)$.

Another interesting property of $\mathrm{GL}_{\mathrm{q}}(1 \mid 1)$ is seen by writing the determinani (2.6) using the exponential representation. Since the variables $\sigma$ and $\tau$ are nilpotent it is straightforward to show that the inverse of $d$ is given by

$d^{-1}=e^{-h u}+\frac{\sigma \tau}{1-(r-u)^{2}}\left(\frac{1}{2}(1-r+u) e^{-h(u+2)}+\frac{1}{2}(1+r-u) e^{-h u}-e^{h(r-2 u-1)}\right)$. Using this expression and (2.9) in the definition of the determinant (2.6), one finds

$$
\operatorname{det}(A)=\left(a-\beta d^{-1} \gamma\right) d^{-1}=e^{h(r-u)} .
$$

The exponent $(r-u)$ is the super-trace of $M$, which is central. Thus we have the general relation

$$
\ln \operatorname{det}(A)=s-\operatorname{tr} \ln (A) .
$$

This coincides with the relation for classical matrices. 


\section{Chapter 3}

\section{The Quantum Algebra $\mathrm{GL}_{\mathrm{q}}(1 \mid 1)$}

In this chapter we determine the algebra of $\mathrm{GL}_{\mathbf{q}}(1 \mid 1)$. We use the method of Wess and Zumino [9] based on an invariant differential calculus. This is a generalization of the work of Woronowicz [8]. Fundamental to this method is an exterior derivative which is nilpotent and obeys the Leibniz rule. Input to the method are the q-relations defined by the R-matrix in (2.1). The elements of a matrix in the group are the group parameters. The method derives the algebra of cenerators of infinitessimal deformations of the group parameters. It also determines the coproduct of the generators.

Here we apply this method to $\mathrm{GL}_{\mathrm{q}}(1 \mid 1)$, starting with the q-relations (2.5). We find the algebra of $\mathrm{GL}_{\mathbf{q}}(1 \mid 1)$ and its subalgebra $\mathrm{SL}_{\mathbf{q}}(1 \mid 1)$, and the coproduct of the generators. Our results are also shown to be consistent with the method of Faddeev, Reshetikhin, and Takhtajan [6, 7].

\subsection{Exterior Derivative and Cartan-Maurer Forms}

We begin by defining the exterior derivative $\delta$. We require $\delta$ to be nilpotent and to obey the (graded) Leibniz rule:

$$
\begin{aligned}
& \delta^{2}=0 \\
& \delta(F G)=(\delta F) G+(-1)^{F} F(\delta G) .
\end{aligned}
$$


Here $(-1)^{F}=+1(-1)$ if $F$ is commuting (anticommuting). This grading is required since $\delta$ is anticommuting.

Next define right-invariant Cartan-Maurer 1-forms in the usual way. For a group element $A$ define:

$$
\Omega=(\delta A) A^{-1}=\left(\begin{array}{cc}
\omega_{1} & \nabla_{+} \\
\nabla_{-} & \omega_{2}
\end{array}\right) .
$$

Here the 1 -forms $\omega_{1}$ and $\omega_{2}$ are anticommuting while $v_{+}$and $v_{-}$are commutin Under right multiplication by a constant group element $A_{0}$ obeying $\delta A_{0}=0$,

$$
\begin{aligned}
& A^{\prime}=A A_{0} \\
& \Omega^{\prime}=\left(\delta A^{\prime}\right)\left(A^{\prime}\right)^{-1}=(\delta A) A^{-1}=\Omega
\end{aligned}
$$

and we see that the Cartan-Maurer forms are right-invariant.

By manipulating the definition of $\Omega$ one can determine the action of the exterior derivative on the group parameters and on the Cartan-Maurer forms. Rearranging (3.2) gives $\delta A=\Omega A$ or for each group parameter

$$
\begin{array}{ll}
\delta a=\omega_{1} a+v_{+} \gamma & \delta \beta=\omega_{1} \beta+v_{+} d \\
\delta \gamma=\omega_{2} \gamma+v_{-} a & \delta d=\omega_{2} d+v_{-} \beta .
\end{array}
$$

Acting on (3.2) with $\delta$ and using (3.1) we have

$$
\delta \Omega=\tilde{\Omega} \Omega \quad \tilde{\Omega}=\left(\begin{array}{cc}
\omega_{1} & -\nabla_{+} \\
-\nabla_{-} & \omega_{2}
\end{array}\right) .
$$

The signs in $\tilde{S}$ are determined by the grading in the Leibniz rule (3.1). Then we find for each 1 -form

$$
\begin{array}{ll}
\delta \omega_{1}=\omega_{1}^{2}-v_{+} v_{-} & \delta v_{+}=\omega_{1} v_{+}-v_{+} \omega_{2} \\
\delta v_{-}=\omega_{2} v_{-}-v_{-} \omega_{1} & \delta \omega_{2}=\omega_{2}^{2}-v_{-} v_{+} .
\end{array}
$$

These are the usual (graded) Cartan-Maurer equations. Note that they are written with the ordering defined by (3.4). This is because we do not yet know how to commute 1-forms; they may obey q-relations which differ from classical (anti)commutators. 
The exterior derivative may be expanded as a sum of the 1-forms multiplying the generators of the algebra:

$$
\delta=\omega_{1} T_{1}+\omega_{2} T_{2}+v_{+} \nabla_{+}+v_{-} \nabla_{-}
$$

The generators $T_{1}$ and $T_{2}$ are commuting and $\nabla_{+}$and $\nabla_{-}$are anticommuting so that overall $\delta$ is anticommuting. From (3.3) one can read off the action of the generators on the group parameters (e.g. $T_{1} a=a$ and $\nabla_{+} a=\gamma$, etc.) The algebra of the generators may be determined using the nilpotecy and Leibniz iule (3.1) obeyed by the exterior derivative. Applying $\delta$ to the above expansion (3.6) gives (sum $\mathrm{i}=1,2$ )

$$
\begin{aligned}
\delta= & \omega_{i} T_{i}+v_{ \pm} \nabla_{ \pm} \\
\delta^{2}= & \left(\delta \omega_{i}\right) T_{i}+\left(\delta v_{ \pm}\right) \nabla_{ \pm}-\omega_{i} \delta T_{i}+v_{ \pm} \delta \nabla_{ \pm} \\
= & \left(\omega_{1}^{2}-v_{+} v_{-}\right) T_{1}-\omega_{1}\left(\omega_{i} T_{i}+v_{ \pm} \nabla_{ \pm}\right) \\
& +\left(\omega_{2}^{2}-v_{-} v_{+}\right) T_{2}-\omega_{2}\left(\omega_{i} T_{i}+v_{ \pm} \nabla_{ \pm}\right) \\
& +\left(\omega_{1} v_{+}-v_{+} \omega_{2}\right) v_{+}+v_{+}\left(\omega_{i} T_{i}+v_{ \pm} \nabla_{ \pm}\right) \\
& +\left(\omega_{2} v_{-}-v_{-} \omega_{1}\right) v_{-}+v_{-}\left(\omega_{i} T_{i}+v_{ \pm} \nabla_{ \pm}\right) \\
& =0 .
\end{aligned}
$$

If we knew how to commute 1-forms, we could rearrange this expansion, with the coefficients of the 1-forms yielding the algebra of generators. Therefore we must determine q-relations between the Cartan-Maurer forms. With this information we can return to this expansion and derive the algebra.

\subsection{Additional q-relations}

First we wish to extend the q-relations between group parameters (2.5) to include q-relations between Cartan-Maurer forms and group parameters. Recall that the Cartan-Maurer forms are right invariant. We shall require the new q-relations to also be right invariant. (This is already true for the relations (2.5)). Under right 
multiplication $A^{\prime}=A A_{0}$ the group parameters transform to

$$
\begin{array}{ll}
a^{\prime}=a a_{0}+\beta \gamma_{0} & \beta^{\prime}=a \beta_{0}+\beta d_{0} \\
\gamma^{\prime}=\gamma a_{0}+d \gamma_{0} & d^{\prime}=\gamma \beta_{0}+d d_{0} .
\end{array}
$$

Under this transformation, $a$ and $\beta$ mix together, and $d$ and $\gamma$ mix. Thus, in order to be right invariant, $a$ and $\beta$ should have the same $\mathrm{q}$-relations with the 1 -forms, and $d$ and $\gamma$ should have the same q-relations (up to $Z_{2}$ grading signs.) We sh:"? consider the following ansatz:

$$
\begin{array}{ll}
a\left(s^{i} \omega_{i}+\sigma^{ \pm} v_{ \pm}\right)=\left(t^{i} \omega_{i}+\tau^{ \pm} v_{ \pm}\right) a & d\left(s^{\prime i} \omega_{i}+\sigma^{\prime \pm} v_{ \pm}\right)=\left(t^{\prime i} \omega_{i}+\tau^{\prime \pm} v_{ \pm}\right) d \\
\beta\left(s^{i} \omega_{i}+\sigma^{ \pm} v_{ \pm}\right)=-\left(t^{i} \omega_{i}+\tau^{ \pm} v_{ \pm}\right) \beta & \gamma\left(s^{i} \omega_{i}+\sigma^{\prime \pm} v_{ \pm}\right)=-\left(t^{i} \omega_{i}+\tau^{ \pm} v_{ \pm}\right) \gamma
\end{array}
$$

where $s^{i}$ etc. define the form mixing. Next, recall that the determinant $D=\operatorname{det} . A$ defined in (2.6) is central, i.e. it commutes with all of the group parameters. We wish to extend this to the algebra with Cartan-Maurer forms:

$$
\begin{gathered}
D \omega_{1,2}=\omega_{1,2} D \\
D v_{ \pm}=v_{ \pm} D .
\end{gathered}
$$

Since $D=a d^{-1}-\beta d^{-1} \gamma d^{-1}, a$ and $d^{-1}$ must have inverse mixing in (3.8), or $a$ and $d$ must have the same mixing, with primed coefficients equal to unprimed ones. Then all group parameters have the same q-relations with the 1-forms (up to grading signs). For a general group parameter $A_{r} \in\{a, \beta, \gamma, d\}$ we write

$$
A_{r}\left(s^{i} \omega_{i}+\sigma^{ \pm} v_{ \pm}\right)=(-1)^{A_{r}}\left(t^{i} \omega_{i}+\tau^{ \pm}\right) A_{r} .
$$

Here $(-1)^{A_{r}}=+1$ for $A_{r}=a$ or $d$ and $(-1)^{A_{r}}=-1$ for $A_{r}=\beta$ or $\gamma$.

We now attempt to determine these relations by applying $\delta$ to the qrelations (2.5). Beginning with the simplest relation $\beta^{2}=0$ we find

$$
\begin{aligned}
\delta \beta^{2} & =(\delta \beta) \beta-\beta(\delta \beta) \\
& =\omega_{1} \beta \beta+v_{+} d \beta-\beta \omega_{1} \beta-\beta v_{+} d \\
& =\left(q v_{+} \beta-\beta v_{+}\right) d \\
& =0 .
\end{aligned}
$$


The first and third terms in the second line vanish since they are proportional to $\beta^{2}$. The third line is obtained from the second using $d \beta=q \beta d$. Since this must vanish for all values of the group parameters, the coefficient of $d$ in the third line must vanish, yielding $\beta v_{+}=q v_{+} \beta$. Following the same procedure on $\gamma^{2}=0$ gives

$$
\delta \gamma^{2}=\left(q v_{-} \gamma-\gamma v_{-}\right) a=0 .
$$

Recalling that all group parameters have the same q-relations with the CartanMaurer forms, we summarize

$$
A_{r} v_{ \pm}=q v_{ \pm} A_{r}
$$

We see that the 1 -forins $v_{ \pm}$obey simple q-relations with the group parameters, with no mixing between 1 -forms.

Acting on the relation $\beta \gamma=-\gamma \beta$ with $\delta$ gives

$$
\delta(\beta \gamma+\gamma \beta)=\left(\omega_{1}-\omega_{2}\right) \beta \gamma+\beta\left(\omega_{1}-\omega_{2}\right) \gamma=0
$$

which is solved by $\left(\omega_{1}-\omega_{2}\right) \beta=-\beta\left(\omega_{1}-\omega_{2}\right)$ or in general

$$
A_{r}\left(\omega_{1}-\omega_{2}\right)=(-1)^{A_{r}}\left(\omega_{1}-\omega_{2}\right) A_{r} .
$$

Here we see that the linear combination $\left(\omega_{1}-\omega_{2}\right)$ is an eigenvector under commutation with eigenvalue 1 . Suppose there is another linear combination with eigenvalue $f$. Define the 1 -forms

$$
\begin{aligned}
& \omega_{Y}=\frac{1}{2}\left(\omega_{1}-\omega_{2}\right) \\
& \omega_{X}=\frac{1}{2}\left((1+\phi) \omega_{1}+(1-\phi) \omega_{2}\right)
\end{aligned}
$$

which obey the q-relations

$$
\begin{aligned}
& A_{r} \omega_{Y}=(-1)^{A_{r}} \omega_{Y} A_{r} \\
& A_{r} \omega_{X}=(-1)^{A_{r}} f \omega_{X} A_{r} .
\end{aligned}
$$

Further application of $\delta$ to the remaining q-relations in (2.5) yields no new information. We are left with two undetermined parameters, $f$ and $\phi$, both related to $\omega_{X}$. 
Next we wish to determine q-relations between the Cartan-Maurer forms themselves. Again we apply $\delta$, this time to the relations (3.10,3.12). Starting with the q-relations between $v_{+}$and $a$ and $d$ gives

$$
\begin{gathered}
\delta\left(a v_{+}-q v_{+} a\right)=q\left(\left(f \omega_{X}+(1-\phi) \omega_{Y}\right) v_{+}-v_{+}\left(f \omega_{X}+(1-\phi) \omega_{Y}\right)\right) a=0 \\
\delta\left(d v_{+}-q v_{+} d\right)=q\left(\left(f \omega_{X}-(1+\phi) \omega_{Y}\right) v_{+}-v_{+}\left(f \omega_{X}-(1+\phi) \omega_{Y}\right)\right) d \\
+q\left(v_{-} v_{+}-v_{+} v_{-}\right) \beta=0 .
\end{gathered}
$$

The coefficients of group parameters must vanish, giving relations between $\omega_{X, Y}$ and $v_{+}$and between $v_{+}$and $v_{-}$. Repeating the procedure starting with the qrelations between $v_{-}$and $a$ and $d$, we arrive at the general relations

$$
\begin{aligned}
& \omega_{i} v_{ \pm}=v_{ \pm} \omega_{i} \quad i=1,2, X, Y \\
& v_{+} v_{-}=v_{-} v_{+} .
\end{aligned}
$$

Note that these relations are independent of $q$ and equivalent to the classical case $q=1$.

The procedure may now be repeated with the relations between wy and $a$ and $d$. Applying $\delta$ gives

$$
\begin{aligned}
& \delta\left(\omega_{Y}-\omega_{Y} a\right)=\left(f\left(\omega_{X} \omega_{Y}+\omega_{Y} \omega_{X}\right)+2(1-\phi) \omega_{Y}^{2}\right) a=0 \\
& \delta\left(d_{Y}-\omega_{Y} d\right)=\left(f\left(\omega_{X} \omega_{Y}+\omega_{Y} \omega_{X}\right)-2(1+\phi) \omega_{Y}^{2}\right) d=0 .
\end{aligned}
$$

Vanishing requires

$$
\begin{aligned}
& \omega_{Y}^{2}=0 \\
& \omega_{X} \omega_{Y}+\omega_{Y} \omega_{X}=0 .
\end{aligned}
$$

Again we find relations independent of $q$ and equivalent to the classical case.

There is one more set of relations, involving $\omega_{X}$. Applying $\delta$ to the relations between $a$ and $\omega_{X}$ gives

$$
\delta\left(a \omega_{X}-f \omega_{X} a\right)=\left(f(f+1) \omega_{X}^{2}+\left(f-q^{2}\right) v_{+} v_{-}\right)=0
$$

This yields the relation

$$
\omega_{X}^{2}=\kappa v_{+} v_{-} \quad \kappa=\frac{q^{2}-f}{f(f+1)} .
$$


Notice that this relation is not equivalent to the classical case where $\omega_{X}^{2}=0$. It involves both the quantum deformation parameter $q$ and the undetermined parameter $f$. There is a final consistency check which will determine $f$. Using the q-relations between between group parameters and Cartan-Maurer forms, we find

$$
\begin{aligned}
A_{r}\left(\omega_{X}^{2}-\kappa v_{+} v_{-}\right) & =\left(f^{2} \omega_{X}^{2}-q^{2} \kappa v_{+} v_{-}\right) A_{r} \\
& =\left(f^{2}-q^{2}\right) \kappa v_{+} v_{-} A_{r} \\
& =\frac{\left(f^{2}-q^{2}\right)\left(q^{2}-f\right)}{f(f+1)} v_{+} v_{-} A_{r} \\
& =0 .
\end{aligned}
$$

There are three solutions for $f$ :

$$
\begin{aligned}
& \text { I) } f=q^{2} \quad \kappa=0 \\
& \text { II) } f=q \quad \kappa=\frac{q-1}{q+1} \\
& \text { III) } f=-q \quad \kappa=\frac{q+1}{q-1} \text {. }
\end{aligned}
$$

The first solution is the simplest, with $\kappa=0$ and (3.15) equivalent to the classical case. The third solution has the wrong limit of (3.12) for $q=1$ and we will disregard this solution.

With the q-relations just derived we may rewrite the Cartan-Maurer equations in a simplified form. They are

$$
\begin{aligned}
& \delta \omega_{Y}=0 \\
& \delta \omega_{X}=(\kappa-1) v_{+} v_{-} \\
& \delta v_{ \pm}= \pm 2 \omega_{Y} v_{ \pm} .
\end{aligned}
$$

For solution $I$ we see once again that the equations agree with the classical case. This is not trie for $\delta \omega_{X}$ for solution $I I$.

\subsection{The Algebra}

The relations between Cartan-Maurer forms provide enough information to determine the algebra of the quantum group. Using these relations to rearrange the 
expansion of $\delta^{2}(3.7)$ we get

$$
\begin{aligned}
\delta^{2}= & v_{+}^{2} \nabla_{+}^{2}+v_{-}^{2} \nabla_{-}^{2}+\omega_{X} \omega_{Y}(X Y-Y X) \\
& +V_{+} \omega_{Y}\left(Y \nabla_{+}-\nabla_{+} Y+2 \nabla_{+}\right)+V_{-} \omega_{Y}\left(Y \nabla_{-}-\nabla_{-} Y+2 \nabla_{-}\right) \\
& +v_{+} \omega_{X}\left(X \nabla_{+}-\nabla_{+} X\right)+v_{-} \omega_{X}\left(X \nabla_{-}-\nabla_{-} X\right) \\
& +v_{+} v_{-}\left(\nabla_{+} \nabla_{-}+\nabla_{-} \nabla_{+}+(\kappa-1) X-\kappa X^{2}\right) .
\end{aligned}
$$

Nilpotency of $\delta$ requires the coefficients of the Cartan-Maurer forms to vanish:

$$
\begin{aligned}
& X Y-Y X=0 \\
& \nabla_{ \pm} Y-Y \nabla_{ \pm}= \pm 2 \nabla_{ \pm} \\
& \nabla_{ \pm}^{2}=0 \\
& \nabla_{ \pm} X-X \nabla_{ \pm}=0 \\
& \nabla_{+} \nabla_{-}+\nabla_{-} \nabla_{+}=(1-\kappa) X+\kappa X^{2} .
\end{aligned}
$$

This is the algebra of the generators of $\mathrm{GL}_{q}(1 \mid 1)$.

There are several features to note about the algebra derived above. First, note that the generator $X$ commutes with all other generators. Thus it may be redefined by any function $X^{\prime}=F(X)$. Specifically it is desirable to simplify the algebra by setting the anticommutator $\left\{\nabla_{+}, \nabla_{-}\right\}_{+}$equal to the redefined generator $X^{\prime}$ :

$$
X^{\prime}=\nabla_{+} \nabla_{-}+\nabla_{-} \nabla_{+}= \begin{cases}X_{I} & \text { solution I } \\ \frac{2}{q+1} X_{I I}+\frac{q-1}{q+1} X_{I I}^{2} & \text { solution II } .\end{cases}
$$

For solution $I$ no redefintion is necessary. For solution $I I$ the above redefinition gives $X_{I}$ in terms of $X_{I I}$. Later we will see that this redefinition is consistent with the coproduct.

With this redefinition, or always for solution $I$, we see that the algebra is independent of $q$. In fact the algebra coincides with the classical algebra. Shortly we will see that this is not true for the coproduct, which does depend on $q$ and is not equivalent to the classical coproduct.

Finally, we note that the generators $\nabla_{ \pm}$and $X$ form a closed subalgebra, given by the last taree lines of (3.18). Recall the quantum determinant defined in 


$$
D=\left(a-\beta d^{-1} \gamma\right) d^{-1} .
$$

Using (3.3) for the action of the exterior derivative on the group parameters and $(3.10,3.12)$ to commute forms, one finds

$$
\delta D=2 \omega_{Y} D .
$$

From the expansion of $\delta(3.6)$, this shows that

$$
X D=0 \quad \nabla_{ \pm} D=0 .
$$

Thus we see that the generators in the subalgebra leave the determinant invariant. Then this subalgebra is $\mathrm{SL}_{\mathbf{q}}(1 \mid 1)$, the algebra of linear transformation with determinant one.

\subsection{The Coproduct}

Now we will consider the action of the generators on functions of the group parameters and on products of functions. This can be done using the previously derived q-relations between Cartan-Maurer forms and group parameters. This study yields the coproduct of the quantum algebra.

First recall the action of the exterior derivative on a product of group parameters $A_{r}, A, \in\{a, \beta, \gamma, d\}$ :

$$
\delta\left(A_{r} A_{\theta}\right)=\left(\delta A_{r}\right) A_{s}+(-1)^{A_{r}} A_{r}\left(\delta A_{s}\right) .
$$

Using the relations $(3.10,3.12)$, one can commute the Cartan-Maurer forms in the second term to the left of $A_{r}$. Equating coefficients of the 1-forms on both sides of the equation gives

$$
\begin{aligned}
X\left(A_{r} A_{\mathbf{s}}\right) & =\left(X A_{r}\right) A_{\mathbf{s}}+f A_{r}\left(X A_{\mathbf{s}}\right) \\
Y\left(A_{r} A_{\mathbf{s}}\right) & =\left(Y A_{r}\right) A_{\mathbf{s}}+A_{r}\left(Y A_{\mathbf{s}}\right) \\
\nabla_{ \pm}\left(A_{r} A_{s}\right) & =\left(\nabla_{ \pm} A_{r}\right) A_{\mathbf{s}}+(-1)^{A_{r}} q A_{r}\left(\nabla_{ \pm} A_{s}\right)
\end{aligned}
$$

where for now we will consider both solutions $f=q, q^{2}$. Repeating this procedure for higher power monomials of the group parameters will determine the action 
on arbitrary functions. It is convenient to simplify this notation by dropping the second term in the product ( $A$, here) and writing the action of the the generators on $A_{r}$ times an arbitrary function $(\cdot)$ to follow:

$$
\begin{aligned}
X A_{r} \cdot & =\left(X A_{r}\right) \cdot+f A_{r} X . \\
Y A_{r^{\prime}} & =\left(Y A_{r}\right) \cdot+A_{r} Y . \\
\nabla_{ \pm} A_{r^{\prime}} & =\left(\nabla_{ \pm} A_{r}\right) \cdot+(-1)^{A_{r}} q A_{r} \nabla_{ \pm} \cdot .
\end{aligned}
$$

We consider in detail first the generator $X$. Consider a monomial of group parameters $a^{m} d^{m} \beta^{i} \gamma^{j}$ where $m, n \geq 0$ and $i, j=0,1$. From above we see that a power of $f$ appears each time we commute $X$ through a group parameter. Repeating the procedure gives

$$
\begin{aligned}
X\left(a^{m} d^{m} \beta^{i} \gamma^{j}\right) & =\left(1+f+f^{2}+\ldots+f^{m+n+i+j}\right)\left(a^{m} d^{m} \beta^{i} \gamma^{j}\right) \\
& =\frac{f^{m+n+i+j}-1}{f-1} a^{m} d^{m} \beta^{i} \gamma^{j} .
\end{aligned}
$$

It is convenent to define a number operator $N$ for the group parameters as follows:

$$
\begin{aligned}
& \left(N A_{r}\right)=A_{r} \\
& N A_{r}=\left(N A_{r}\right) \cdot+A_{r} N \\
& \left(N a^{m} d^{m} \beta^{i} \gamma^{j}\right)=(m+n+i+j)\left(a^{m} d^{m} \beta^{i} \gamma^{j}\right) .
\end{aligned}
$$

Then the generator $X$ and $N$ are related by

$$
\begin{aligned}
X & =\frac{f^{N}-1}{f-1} \\
f^{N} & =1+(f-1) X .
\end{aligned}
$$

Now any funtion $F$ of the group parameters can be written as a sum of monomials. By repeating the above procedures on a product of monomials, we find the action of $X$ on products of functions to be

$$
\begin{aligned}
X F \cdot & =(X F) \cdot+\left(f^{N} F\right) X . \\
& =(X F) \cdot+((1+(f-1) X) F) X .
\end{aligned}
$$

The result for the generators $\nabla_{ \pm}$is obtained in a similar manner. Here instead of a factor of $f$ (which is equal to $q$ or $q^{2}$ ) there is always a factor of $q$ 
when commuting through a group parameter. On a product of functions

$$
\nabla_{ \pm} F \cdot=\left(\nabla_{ \pm} F\right) \cdot+(-1)^{F}\left(q^{N} F\right) \nabla_{ \pm} \cdot
$$

Finally for the generator $Y$ we obtain the simple result

$$
Y F \cdot=(Y F) \cdot+F Y \cdot
$$

In this final case note that there are no factors of $q$. In fact the action of $Y$ is equivalent to the classical case.

These results lead to the coproduct of the quantum algebra. If $A$ is the universal enveloping algebra, then the coproduct $\Delta$ is a map to the tensor product of $\mathcal{A}$ :

$$
\begin{aligned}
\Delta: \mathcal{A} & \rightarrow \mathcal{A} \otimes \mathcal{A} \\
F & \mapsto \Delta(F) \quad F \in \mathcal{A} .
\end{aligned}
$$

The map must be a homomorphism of the algebra:

$$
\Delta(F G)=\Delta(F) \Delta(G) \quad F, G \in \mathcal{A} .
$$

It is straightforward to read off the coproduct from the results in equitions (3.1S3.21). For both solutions we have

$$
\begin{aligned}
\Delta\left(\nabla_{ \pm}\right) & =\nabla_{ \pm} \otimes 1+q^{N} \otimes \nabla_{ \pm} \\
\Delta(Y) & =Y \otimes 1+1 \otimes Y \\
\Delta\left(X_{I}\right) & =X_{I} \otimes 1+q^{2 N} \otimes X_{I} \\
\Delta\left(X_{I I}\right) & =X_{I I} \otimes 1+q^{N} \otimes X
\end{aligned}
$$

where in terms of $X$

$$
q^{N}= \begin{cases}\left(1+\left(q^{2}-1\right) X_{l}\right)^{\frac{1}{2}} & \text { solution } I \\ 1+(q-1) X_{I I} & \text { solution } I I .\end{cases}
$$

Firally, we note that the redefinition of $X$ for the two solutions for $f$ :

$$
X_{I}=\frac{2}{q+1} X_{I I}+\frac{q-1}{q+1} X_{I I}^{2}
$$

is consistent with the coproduct:

$$
\Delta\left(X_{I}\right)=\frac{2}{q+1} \Delta\left(X_{I I}\right)+\frac{q-1}{q+1} \Delta\left(X_{I I}\right)^{2} .
$$


This shows that both solutions for $X$ are in the same universal enveloping algebra, and are in fact reparameterizations of each other. For the remainder of the discussion we will take solution $I$ with $f=q^{2}$ and set $X=X_{I}$.

\subsection{Method of Faddeev, Reshetikhin, and Takhtajan}

It is instructive to compare the results based on the differential calculus to those of Faddeev, Reshetikhin, and Takhtajan [6, 7]. Their method is based on the $P$ matrix (2.4) used earlier in defining the quantum group. Define the matrix $I^{\prime}$ by

$$
R=P R F=\left(\begin{array}{cccc}
q & 0 & 0 & 0 \\
0 & 1 & \lambda & 0 \\
0 & 0 & 1 & 0 \\
0 & 0 & 0 & q^{-1}
\end{array}\right)
$$

Here $P$ is the permutation matrix acting on the tensor product of vector spaces $\mathcal{V} \otimes \mathcal{V}$ according to $P(u \otimes v)=v \otimes u$ for $u, v \in \mathcal{V}$, or:

$$
P=\left(\begin{array}{llll}
1 & 0 & 0 & 0 \\
0 & 0 & 1 & 0 \\
0 & 1 & 0 & 0 \\
0 & 0 & 0 & 1
\end{array}\right)
$$

Recall also that $\lambda=q-q^{-1}$. Nexi define the upper- and lower-triangular $2 \times 2$ matrices

$$
L^{+}=\left(\begin{array}{cc}
U_{+} & \lambda \chi_{+} \\
0 & W_{+}
\end{array}\right) \quad L^{-}=\left(\begin{array}{cc}
U_{-} & 0 \\
-\lambda \chi_{-} & W_{-}
\end{array}\right) .
$$


Finally define the $4 \times 4$ matrices $L_{1,2}$ which act on $\mathcal{V} \otimes \mathcal{V}$ by $L_{1}^{ \pm}=L^{ \pm} \otimes 1$ and $L_{2}^{ \pm}=1 \otimes L^{ \pm}$. These matrices are

$$
\begin{array}{rlr}
L_{1}^{+} & =\left(\begin{array}{cccc}
U_{+} & 0 & \lambda \chi_{+} & 0 \\
0 & U_{+} & 0 & \lambda \chi_{+} \\
0 & 0 & W_{+} & 0 \\
0 & 0 & 0 & W_{+}
\end{array}\right) & L_{1}^{-}=\left(\begin{array}{cccc}
U_{-} & 0 & 0 & 0 \\
0 & U_{-} & 0 & 0 \\
-\lambda x_{-} & 0 & W_{-} & 0 \\
0 & -\lambda \chi_{-} & 0 & W_{-}
\end{array}\right) \\
L_{2}^{+}=\left(\begin{array}{cccc}
U_{+} & \lambda \chi_{+} & 0 & 0 \\
0 & W_{+} & 0 & 0 \\
0 & 0 & U_{+} & -\lambda \chi_{+} \\
0 & 0 & 0 & W_{+}
\end{array}\right) & L_{2}^{-}=\left(\begin{array}{cccc}
U_{-} & 0 & 0 & 0 \\
-\lambda \chi_{-} & W_{-} & 0 & 0 \\
0 & 0 & U_{-} & 0 \\
0 & 0 & \lambda \chi_{-} & W_{-}
\end{array}\right) .
\end{array}
$$

Here we have used index ordering to allow simple matrix multiplication (see appendix).

The method of Faddeev, Reshetikhin, and Takhtajan uses these matrices to determine the quantum algebra and coproduct. The equations

$$
\begin{aligned}
& R^{\prime} L_{1}^{ \pm} L_{2}^{ \pm}=L_{2}^{ \pm} L_{1}^{ \pm} R^{\prime} \\
& R^{\prime} L_{1}^{+} L_{2}^{-}=L_{2}^{-} L_{1}^{+} R^{\prime}
\end{aligned}
$$

yield relations on $U_{ \pm}, W_{ \pm}$, and $\chi_{ \pm}$. These relations lead to the quantum algebra. One finds that $U_{ \pm}$and $W_{ \pm}$all commute with each other, and also that the combinations $U_{+} U_{-}$and $W_{+} W_{-}$commute with $\chi_{ \pm}$. Then we may take these combinations to be proportional to the identity and define

$$
\begin{array}{ll}
U_{+}=U & U_{-}=U^{-1} \\
W_{+}=W & W_{-}=W^{-1} .
\end{array}
$$

Then the remaining relations are

$$
\begin{aligned}
& \chi_{ \pm} U=q^{ \pm 1} U \chi_{ \pm} \\
& \chi_{ \pm} W=q^{ \pm 1} W \chi_{ \pm} \\
& \chi_{+} \chi_{-}+\chi-\chi_{+}=\lambda^{-1}\left(U^{-1} W-U W^{-1}\right) .
\end{aligned}
$$

These relations determine the quantum algebra. 
The coproduct is determined by

$$
\Delta\left(L^{ \pm}\right)=L^{ \pm} \dot{\otimes} L^{ \pm}
$$

where $\dot{\otimes}$ denotes tensor product combined with usual matrix multiplication. Using the matrices $L^{ \pm}$defined above one gets

$$
\begin{aligned}
\Delta(U) & =U \otimes U \quad \Delta(W)=W \otimes W \\
\Delta\left(\chi_{+}\right) & =\chi_{+} \otimes W+U \otimes \chi_{+} \\
\Delta\left(\chi_{-}\right) & =\chi_{-} \otimes U^{-1}+W^{-1} \otimes \chi_{-}
\end{aligned}
$$

where we have used the above definitions of $U$ and $W$.

These results may now be compared to the algebra and coproduct derived earlier using the differential calculus. We can do this by writing one set of generators as functions of the other set. Because of the rather simple form of the algebra, there are many such reparameterizations which yield the same form of the algebra. However, the coproduct will give a unique relation between the two formulations. Consider the combinations $W^{-1} \chi_{+}$and $U_{\chi_{-}}$. Using the coproducts (3.24) we find

$$
\begin{aligned}
\Delta\left(W^{-1} \chi_{+}\right) & =W^{-1} \chi_{+} \otimes 1+U W^{-1} \otimes W^{-1} \chi_{+} \\
\Delta\left(U_{\chi_{-}}\right) & =U \chi_{-} \otimes 1+U W^{-1} \otimes U \chi_{-} .
\end{aligned}
$$

Comparison with the coproducts (3.22) indicates that $W^{-1} \chi_{+} \propto \nabla_{+}$and $U_{-} \propto$ $\nabla_{\text {. }}$. Including normalization factors to get the same algebra, we find that the first set of generators can be written in terms of the new ones as

$$
\begin{array}{ll}
\nabla_{+}=q^{-1} W^{-1} \chi_{+} & X=\frac{U^{2} W^{-2}-1}{q^{2}-1} \\
\nabla_{-}=q^{-1} U \chi_{-} & q^{N}=U W^{-1} .
\end{array}
$$

For the inverse relations, note that

$$
q^{-\frac{1}{2} Y} \nabla_{ \pm}=q^{\mp 1} \nabla_{ \pm} q^{-\frac{1}{2} Y}
$$

where we have used the commutation relations for $Y$ in (3.18). Then it is easy to see that

$$
\begin{array}{ll}
\chi_{+}=q q^{-\frac{1}{2} Y-\frac{1}{2} N} \nabla_{+} & U=q^{-\frac{1}{2} Y+\frac{1}{2} N} \\
\chi_{-}=q q^{\frac{1}{2} Y-\frac{1}{2} N} \nabla_{-} & W=q^{-\frac{1}{2} Y-\frac{1}{2} N}
\end{array}
$$


are the correct reparameterizations. Substitution of these relations into one form of the algebra leads to the other form. Thus both methods yield the same quantum universal enveloping algebra. 


\section{Chapter 4}

\section{The Quantum Super-Plane}

In this chapter we discuss the quantum super-plane. This follows an approach to quantum groups first suggested by Y. Kobyzev and developed by Manin [5]. By detining coordinates and differentials which ubey q-relations (i.e. they do not obey classical commutations rules), and demanding covariance, one obtains the previous q-relations on the elements of matrices which act on the plane coordinates. The converse is also true: the q-relations on the matrix elements imply the q-relations on coordinates and differentials.

We will follow this approach for the quantum group $\mathrm{GL}_{\mathbf{q}}(1 \mid 1)$. First we will show the equivalence of group q-relations and super-plane q-relations. Then we will develop a covariant differential calculus on the super-plane. Finally, we obtain a representation of the generators of $\mathrm{GL}_{\mathbf{q}}(1 \mid 1)$ in terms of the super-plane coordinates.

\subsection{Coordinate and Differential q-relations}

Wo begin by defining the super-plane coordinate vector $V$ and differential vector $U$ with components

$$
V=\left(\begin{array}{l}
x \\
\xi
\end{array}\right) \quad U=\left(\begin{array}{l}
\eta \\
y
\end{array}\right) .
$$


The corresponding components of $V$ and $U$ have opposite Grassmann parity. Following Manin we impose the q-relations

$$
\begin{array}{ll}
x \xi=q \xi x & \xi^{2}=0 \\
y \eta=q \eta y & \eta^{2}=0 .
\end{array}
$$

(The nilpotency conditions on $\xi$ and $\eta$ are the same as in the classical case.) These vectors are acted on by matrices $A$ with elements

$$
A=\left(\begin{array}{ll}
a & \beta \\
\gamma & d
\end{array}\right)
$$

where we do not yet know the commutation relations between $a, \beta, \gamma$, and $d$. The matrix elements all commute or anticommute with the vector components, depending on Grassmann parity. The vectors transform under left-multiplication by $A$ :

$$
V^{\prime}=A V \quad U^{\prime}=A U
$$

with new components

$$
\begin{array}{ll}
x^{\prime}=a x+\beta \xi & \eta^{\prime}=a \eta+\beta y \\
\xi^{\prime}=\gamma x+d \xi & y^{\prime}=\gamma \eta+d y .
\end{array}
$$

We now impose the condition that the q-relations (4.2) are covariant, i.e. that the transformed components satisfy the same q-relations. This leads to the familiar q-relations on the matrix elements. For example, the products

$$
\begin{aligned}
& x^{\prime} \xi^{\prime}=a \gamma x^{2}+\left(a d-q^{-1} \beta \gamma\right) x \xi \\
& \xi^{\prime} x^{\prime}=\gamma a x^{2}+\left(\gamma \beta-q^{-1} d a\right) x \xi
\end{aligned}
$$

can be plugged into $x^{\prime} \xi^{\prime}=q \xi^{\prime} x^{\prime}$. Equating coefficients of $x^{2}$ and $x \xi$ gives

$$
a \gamma=q \gamma a \quad a d-q^{-1} \gamma \beta=q \gamma \beta+d a .
$$

Summarizing, the super-plane relations and the matrix element relations they imply are

$$
\begin{array}{rlll}
x^{\prime} \xi^{\prime}=q \xi^{\prime} x^{\prime} & \Longrightarrow & a \gamma=q \gamma a & a d-q^{-1} \beta \gamma=d a+q \gamma \beta \\
y^{\prime} \eta^{\prime}=q \eta^{\prime} y^{\prime} & \Longrightarrow & d \beta=q \beta d & a d+q \beta \gamma=d a-q^{-1} \gamma \beta \\
\dot{\zeta}^{2}=0 & \Longrightarrow & d \gamma=q \gamma d & \gamma^{2}=0 \\
\eta^{2}=0 & \Longrightarrow & a \beta=q \beta a & \beta^{2}=0 .
\end{array}
$$


Finally, the last equations in the first two lines imply

$$
a d-d a=-\left(q-q^{-1}\right) \beta \gamma \quad \beta \gamma=-\gamma \beta
$$

and we see that we have reproduced the q-relations between group parameters (2.5) as defined by the R-matrix method.

The converse of these arguments is also true. Given the q-relations between group parameters, one can find bilinear q-relations on the super-plane coordiantes and differentials which are preserved by a group rotation. This means that if the bilinear relations are given by

$$
F_{i}(x, \xi, \eta, y)=0
$$

then the transformed relations also vanish:

$$
F_{i}\left(x^{\prime}, \xi^{\prime}, \eta^{\prime}, y^{\prime}\right)=0
$$

where the primed components are given by (4.4). In fact it will be seen that this final function is a linear combination of the previous ones:

$$
F_{i}\left(x^{\prime}, \xi^{\prime}, \eta^{\prime}, y^{\prime}\right)=\sum_{j} r_{i j}(a, \beta, \gamma, d) F_{j}(x, \xi, \eta, y) .
$$

Such a set of preserved relations is said to be covariant under the quantum group action.

It is easy to check these relations for the quantum super-plane, since we already know what they should be. However it is instructive to examine at least one case in detail since these techniques will be used later. Let us consider bilinears in the coordinates $x$ and $\xi$. Using the primed components (4.4) and the q-relations on group parameters to rearrange terms we get for $x$ and $\xi$

$$
\begin{aligned}
x^{\prime} \xi^{\prime} & =\gamma a q x^{2}+a d x \xi-\beta \gamma \xi x+\beta d \xi^{2} \\
\xi^{\prime} x^{\prime} & =\gamma a x^{2}+a d \xi x+\beta \gamma(\lambda \xi x-x \xi)-\beta d q \xi^{2} \\
\xi^{\prime 2} & =\gamma d(x \xi-q \xi x)+d^{2} \xi^{2}
\end{aligned}
$$

and it is easily seen that covariant combinations are

$$
\begin{aligned}
x^{\prime} \xi^{\prime}-q \xi^{\prime} x^{\prime} & =(a d-\beta \gamma)(x \xi-q \xi x)+\beta d\left(1+q^{2}\right) \xi^{2} \\
\xi^{2} & =\gamma d(x \xi-q \xi x)+d^{2} \xi^{2} .
\end{aligned}
$$


Proceeding similarly with the differentials shows that

$$
\begin{aligned}
y^{\prime} \eta^{\prime}-q \eta^{\prime} y^{\prime} & =\left(a d-q^{-1} \beta \gamma\right)(y \eta-q \eta y)+\gamma a\left(1+q^{2}\right) \eta^{2} \\
\eta^{2} & =a^{2} \eta^{2}+\beta a(y \eta-q \eta y)
\end{aligned}
$$

are covariant. Summarizing, we may write these covariant combinations as

$$
\begin{array}{ll}
F_{1}=x \xi-q \xi x=0 & F_{2}=\xi^{2}=0 \\
F_{3}=y \eta-q \eta y=0 & F_{4}=\eta^{2}=0 .
\end{array}
$$

We have recovered the relations with which we began this discussion, and we see that the arrows in (4.5) run in both directions.

Finally, we note that the q-relations on coordinates and differentials can be derived from the R-matrix. Define two column vectors in the tensor product of the vector space acted on by the quantum matrices:

$$
\left(V_{1} \otimes V_{2}\right)=\left(\begin{array}{c}
x^{2} \\
x \xi \\
\xi x \\
\xi^{2}
\end{array}\right) \quad\left(U_{1} \otimes U_{2}\right)=\left(\begin{array}{c}
\eta^{2} \\
-\eta y \\
y \eta \\
-y^{2}
\end{array}\right) .
$$

(The signs in $\left(U_{1} \otimes U_{2}\right)$ are needed for covariance, as discussed for the vector $U$ in the next section.) Then using $R$ from (2.4) and $P$ from the previous chapter define the matrix $\hat{R}$ :

$$
\hat{R}=P R=\left(\begin{array}{cccc}
q & 0 & 0 & 0 \\
0 & \lambda & 1 & 0 \\
0 & 1 & 0 & 0 \\
0 & 0 & 0 & -q^{-1}
\end{array}\right)
$$

where $\lambda=q-q^{-1}$. The the equations

$$
(\hat{R}-q 1)\left(V_{1} \otimes V_{2}\right)=0 \quad\left(\hat{R}+q^{-1} 1\right)\left(U_{1} \otimes U_{2}\right)=0
$$

determine the coordinate and differential q-relations (4.2).

\subsection{Super-Plane Exterior Derivative}

Next we wish to formulate a covariant differential calculus on the quantum superplane, involving the derivatives of the coordinates and their differentials. As with 
the quantum algebra, we begin by defining an exterior derivative $\delta$ which is nilpotent and obeys the graded Leibniz rule (3.1). Expand $\delta$ in products of differentials with derivatives:

$$
\delta=\delta x \partial_{x}+\delta \xi \partial_{\xi}
$$

Here $\delta x$ and $\partial_{\xi}$ are Grassmann odd and $\delta \xi$ and $\partial_{x}$ are Grassmann even so that overall $\delta$ is odd. Comparing to the differential vector $U$ in (4.1), one might expect to identify $\delta x=\eta$ and $\delta \xi=y$. However, this is not quite correct. We wish a calculus which is covariant under the group action defined in (4.3). Applying $\delta$ to the transformed components of $V$ gives

$$
\begin{gathered}
\delta x^{\prime}=a \delta x-\beta \delta \xi \\
\delta \xi^{\prime}=-\gamma \delta x+d \delta \xi .
\end{gathered}
$$

Now if we define

$$
\eta=\delta x \quad y=-\delta \xi
$$

as the components of $U$, we find that this differential is also covariant under the group action (4.3).

The exterior derivative is now written

$$
\delta=\eta \partial_{x}-y \partial_{\xi}
$$

As with the algebra, the general properties of $\delta$ allow one to determine sorne information on the algebra of the derivatives. Checking nilpotency gives

$$
\begin{aligned}
\delta^{2} & =-\eta\left(\eta \partial_{x}-y \partial_{\xi}\right) \partial_{x}-y\left(\eta \partial_{x}-y \partial_{\xi}\right) \partial_{\xi} \\
& =\eta y\left(\partial_{\xi} \partial_{x}-q \partial_{x} \partial_{\xi}\right)+y^{2} \partial_{\xi}^{2} \\
& =0
\end{aligned}
$$

where we have used (4.2). The coefficients of the forms must vanish, leaving

$$
\partial_{\xi} \partial_{x}=q \partial_{x} \partial_{\xi} \quad \partial_{\xi}^{2}=0
$$

as the basic algebra of the derivatives. 


\subsection{Additional q-relations}

We wish to extend the derivative algebra to include the coordinates $x$ and $\xi$. As with the group algebra, we will use $\delta$ and the Leibniz rule to determine this. This will require that we know how to commute differentials with coordinates. Some information can be obtained from the basic coordinate q-relations in (4.6). Applying $\delta$ gives

$$
\begin{aligned}
\delta(x \xi-q \xi x) & =\eta \xi-x y+q y x+q \xi \eta=0 \\
\delta\left(\xi^{2}\right) & =-y \xi+\xi y=0 .
\end{aligned}
$$

The second relation tells us how to commute $y$ and $\xi$. However, the first relation does not provide all the information needed for the rest of the components. More assumptions will be needed for these relations.

The first line of (4.8) indicates that there may be some mixing between the coordinate-differential bilinears. Suppose that one relation takes the form

$$
x y=r y x+r^{\prime} \eta \xi .
$$

One of the parameters may be eliminated by requiring that $\delta$ applied to this relation be consistent with $y \eta=q \eta y$. This fixes $r^{\prime}=1-q r$. Finally, adding a multiple of $\delta F_{1}$ gives a similar relation for $\xi$ and $\eta$, leaving

$$
\begin{aligned}
& x y=r y x+(1-q r) \eta \xi \\
& \xi \eta=-r \eta \xi+\left(q^{-1} r-1\right) y x .
\end{aligned}
$$

The parameter $r$ may be determined by a consistency check. Using the second relation twice to commute $\eta$ through $\xi^{2}$ and recalling that $\xi^{2}=0$ gives

$$
\xi^{2} \eta=\left(1-q^{-1} r\right)(q r-1) y \xi x=0
$$

which is solved by $r=q^{ \pm 1}$. Plugging into the above relations we have for $r=q$

$$
\begin{aligned}
& x y=q y x+\left(1-q^{2}\right) \eta \xi \\
& \xi \eta=-q \eta \xi
\end{aligned}
$$

and for $r=q^{-1}$

$$
\begin{aligned}
& x y=q^{-1} y x \\
& \xi \eta=-q^{-1} \eta \xi+\left(q^{-2}-1\right) y x
\end{aligned}
$$


The relations between these bilinears have been completely determined.

Relations between $x$ and $\eta$ are still needed. To determine these recall the covariance of $F_{1-4}$ in (4.6) under the group action as discussed earlier. Requiring covariance for the new relations will give the missing relations. First, for $r=q$ consider

$$
\begin{aligned}
\xi^{\prime} \eta^{\prime}+q \eta^{\prime} \xi^{\prime}= & a d(\xi \eta+q \eta \xi)+\beta d q(y \xi-\xi y) \\
& \quad+\gamma \beta\left(x y-q y x+\left(q^{2}-1\right) \eta \xi\right)+\gamma a\left(x \eta-q^{2} \eta x\right) \\
= & 0 .
\end{aligned}
$$

This vanishes if the new relations are valid and if $x \eta=q^{2} \eta x$. For $r=q^{-1}$ take

$$
\begin{aligned}
x^{\prime} y^{\prime}-q^{-1} y^{\prime} x^{\prime}= & a d\left(x y-q^{-1} y x\right)+\beta d(\xi y-y \xi) \\
& \quad+\gamma \beta\left(\xi \eta+q^{-1} \eta \xi+\left(1-q^{-2}\right) y x\right)+\gamma a\left(q x \eta-q^{-1} \eta x\right) \\
= & 0 .
\end{aligned}
$$

which will vanish if $x \eta=q^{-2} \eta x$. All of the needed relations have been determined. Summarizing, we have for $r=q$ :

$$
\begin{array}{ll}
x y=q y x+\left(1-q^{2}\right) \eta \xi & x \eta=q^{2} \eta x \\
\xi \eta=-q \eta \xi & \xi y=y \xi
\end{array}
$$

and for $r=q^{-1}$ :

$$
\begin{array}{ll}
x y=q^{-1} y x & x \eta=q^{-2} \eta x \\
\xi \eta=-q^{-1} \eta \xi+\left(q^{-2}-1\right) y x & \xi y=y \xi .
\end{array}
$$

Finally, although we have only shown covariance for some of the relations, it can be shown that for each case $r=q^{ \pm 1}$ all of the relations listed here are covariant.

\subsection{Derivative q-relations}

Now we can determine the operator relations between derivatives and coordinates. We will use our previous notation for operator equations where arbitrary functions 
to the right are indicated by $(\cdot)$. Applying $\delta$ to $x \cdot$ gives

$$
\begin{aligned}
\delta x \cdot & =\eta \partial_{x} x \cdot-y \partial_{\xi} t \\
& =(\delta x) \cdot+x \delta . \\
& =\eta \cdot+x \eta \partial_{x} \cdot-x y \partial_{\xi} .
\end{aligned}
$$

where the second line is a result of the Leibniz rule for $\delta$. Using the q-relations $(4.9,4.10)$ we can commute the differentials to the left in the third line. Comparing to the first line gives the desired derivative operator relations. Similarly, for $\xi$. we have

$$
\begin{aligned}
\delta \xi \cdot & =\eta \partial_{x} \xi \cdot-y \partial_{\xi} \xi \\
& =(\delta \xi) \cdot-\xi \delta . \\
& =-y \cdot-\xi \eta \partial_{x} \cdot+\xi y \partial_{\xi} \cdot .
\end{aligned}
$$

Now using the q-relations for $r=q$ we find

$$
\begin{array}{ll}
\partial_{x} x \cdot=1 \cdot+q^{2} x \partial_{x} \cdot+\left(q^{2}-1\right) \xi \partial_{\xi} . & \partial_{x} \xi \cdot=q \xi \partial_{x} . \\
\partial_{\xi} \xi \cdot=1 \cdot-\xi \partial_{\xi} . & \partial_{\xi} x \cdot=q x \partial_{\xi} .
\end{array}
$$

and for $r=q^{-1}$

$$
\begin{array}{ll}
\partial_{x} x=1 \cdot+q^{-2} x \partial_{x} \cdot & \partial_{x} \xi \cdot=q^{-1} \xi \partial_{x} . \\
\partial_{\xi} \xi=1 \cdot-\xi \partial_{\xi} \cdot+\left(q^{-2}-1\right) x \partial_{x} . & \partial_{\xi} x=q^{-1} x \partial_{\xi} .
\end{array}
$$

These two sets of relations are the desired derivative operator equations.

There are also similar operator relations between the derivatives and the differentials. They are obtained by acting with the derivatives on the q-relations between differentials and coordinates. For example, for $r=q$ one finds

$$
\begin{aligned}
& \partial_{x}(\eta x)=q^{-2} \eta \quad \partial_{\xi}(\eta x)=0 \\
& \partial_{x}(\eta \xi)=0 \quad \partial_{\xi}(\eta \xi)=-q^{-1} \eta \\
& \partial_{x}(y x)=q^{-1} y \quad \partial_{\xi}(y x)=\left(q^{-2}-1\right) \eta \\
& \partial_{x}(y \xi)=0 \quad \partial_{\xi}(y \xi)=y .
\end{aligned}
$$

Then these imply the operator relations

$$
\begin{array}{ll}
\partial_{x} \eta \cdot=q^{-2} \eta \partial_{x} . & \partial_{\xi} \eta \cdot=-q^{-1} \eta \partial_{\xi} . \\
\partial_{x} y \cdot=q^{-1} y \partial_{x} . & \partial_{\xi} y \cdot=y \partial_{\xi} \cdot+\left(q^{-2}-1\right) \eta \partial_{x} \cdot .
\end{array}
$$


Repeating the procedure for $r=q^{-1}$ gives

$$
\begin{array}{ll}
\partial_{x} \eta \cdot=q^{2} \eta \partial_{x}-\left(q^{2}-1\right) y \partial_{\xi} . & \partial_{\xi} \eta \cdot=-q \eta \partial_{\xi} . \\
\partial_{x} y=q y \partial_{x} . & \partial_{\xi} y \cdot=y \partial_{\xi} \cdot .
\end{array}
$$

At this point we have determined all possible operator relations between the coordinates, differentials, and derivatives.

\subsection{Representation of Derivatives}

Next the derivative-coordinate operator relations $(4.11,4.12)$ can be used repeatedly to find similar relations between the derivatives and monomials in the coodinates. Then an arbitrary function can be written as a power series in the monomials. In fact, since $\xi^{2}=0$, the most general function in $x$ and $\xi$ can be written

$$
F(x, \xi)=F_{0}(x)+\xi F_{1}(x)
$$

and if $F(x, \xi)$ has definite Grassmann parity then $(-1)^{F}=(-1)^{F_{0}}=-(-1)^{F_{1}}$.

We take first the solution $r=q$. Using the operator relations (4.11) on a power of $x$ gives

$$
\begin{aligned}
\partial_{x} x^{n} \cdot & =\sum_{l=0}^{n-1} q^{2 l} x^{n-1}\left(1+\left(q^{2}-1\right) \xi \partial_{\xi}\right) \cdot+q^{2 n} x^{n} \partial_{x} \cdot \\
& =x^{-1} \frac{q^{2 n}-1}{q^{2}-1} x^{n}\left(1+\left(q^{-2}-1\right) \xi \partial_{\xi}\right) \cdot+q^{2 n} x^{n} \partial_{x} \cdot
\end{aligned}
$$

Then for an arbitrary function of $x$ we have

$$
\partial_{x} F_{0}(x) \cdot=x^{-1} \frac{F_{0}\left(q^{2} x\right)-F_{0}(x)}{q^{2}-1}\left(1+\left(q^{2}-1\right) \xi \partial_{\xi}\right) \cdot+F_{0}\left(q^{2} x\right) \partial_{x} \cdot
$$

It is easy to extend this to a function multiplying $\xi$ :

$$
\begin{aligned}
\partial_{x} \xi F_{1}(x) \cdot & =q \xi \partial_{x} F_{1}(x) \cdot \\
& =x^{-1} q^{2} \xi \frac{F_{1}\left(q^{2} x\right)-F_{1}(x)}{q^{2}-1}\left(1+\left(q^{2}-1\right) \xi \partial_{\xi}\right)+q \xi F_{1}\left(q^{2} x\right) \partial_{x} \cdot .
\end{aligned}
$$

Comparing the results for these two cases shows that for an arbitrary function $F(x, \xi)$

$$
\partial_{x} F(x, \xi) \cdot=x^{-1} \frac{F\left(q^{2} x, q^{2} \xi\right)-F\left(x, q^{2} \xi\right)}{q^{2}-1}\left(1+\left(q^{2}-1\right) \xi \partial_{\xi}\right) \cdot+F\left(q^{2} x, q \xi\right) \partial_{x}
$$


The results for $\partial_{\xi}$ are somewhat sirnpler. Repeating the above arguments we find

$$
\begin{aligned}
\partial_{\xi} x^{n} \cdot & =q^{n} x^{n} \partial_{\xi} . \\
\partial_{\xi} F_{0}(x) \cdot & =(-1)^{F_{0}} F_{0}(q x) \partial_{\xi} . \\
\partial_{\xi} \xi F_{1}(x) \cdot & =F_{1}(x)-(-1)^{F_{1}} \xi F_{1}(q x) \partial_{\xi} \cdot .
\end{aligned}
$$

Then for a general function as in (4.15)

$$
\partial_{\xi} F(x, \xi) \cdot=F_{1}(x) \cdot+(-1)^{F} F(q x, \xi) \partial_{\xi} .
$$

is the operator equation for $\partial_{\xi}$.

If there re no functions to the right of $F$ in the operator equations (4.16) and (4.17), these equations give the action of $\partial$ on $F$. We find for $F=F_{0}+\xi F_{1}$ as above

$$
\begin{aligned}
& \partial_{x} F(x, \xi)=x^{-1} \frac{F\left(q^{2} x, q^{2} \xi\right)-F\left(x, q^{2} \xi\right)}{q^{2}-1} \\
& \partial_{\xi} F(x, \xi)=F_{1}(x) .
\end{aligned}
$$

The operator equations may be rewritten in terms of these actions as

$$
\begin{aligned}
& \partial_{x} F(x, \xi) \cdot=\left(\partial_{x} F(x, \xi)\right)\left(1+\left(q^{2}-1\right) \xi \partial_{\xi}\right) \cdot+F\left(q^{2} x, q \xi\right) \partial_{x} \cdot . \\
& \partial_{\xi} F(x, \xi) \cdot=\left(\partial_{\xi} F(x, \xi)\right) \cdot+(-1)^{F} F(q x, \xi) \partial_{\xi} \cdot
\end{aligned}
$$

This form of the equations is suggestive of a coproduct, for it gives the action of the derivative on a product of functions. We will dicuss this shortly.

The entire procedure may be repeated for the solution $r=q^{-1}$. The action of the derivatives is

$$
\begin{aligned}
& \partial_{x} F(x, \xi)=x^{-1} \frac{F\left(q^{-2} x, \xi\right)-F(x, \xi)}{q^{-2}-1} \\
& \partial_{\xi} F(x, \xi)=F_{1}\left(q^{-2} x\right)
\end{aligned}
$$

and the operator equations are

$$
\begin{aligned}
& \partial_{x} F(x, \xi) \cdot=\left(\partial_{x} F(x, \xi)\right) \cdot+F\left(q^{-2} x, q^{-1} \xi\right) \partial_{x} \cdot \\
& \partial_{\xi} F(x, \xi)=\left(\partial_{\xi} F(x, \xi)\right)\left(1+\left(q^{-2}-1\right) x \partial_{x}\right) \cdot+(-1)^{F} F\left(q^{-1} x, \xi\right) \partial_{\xi} \cdot
\end{aligned}
$$

We see that the mixing of derivatives is reversed between the two solutions, and $q$ is replaced by $q^{-1}$. 
It is useful to introduce number operators for the coordinates $x$ and $\xi$. They will allow the derivatives to be written in an illuminating operator form. Shortly they will also be used to write a representation of the algebra $G_{\mathbf{q}}(1 \mid 1)$. Define the number operators action on coordinates as

$$
\begin{array}{ll}
n_{x} x=x & n_{\xi} x=0 \\
n_{x} \xi=0 & n_{\xi} \xi=\xi
\end{array}
$$

and assume that they obey the usual Leibniz rule, e.g.

$$
n_{i} F \cdot=\left(n_{i} F\right) \cdot+F n_{i} .
$$

Then on a monomial

$$
\begin{aligned}
& n_{x} \xi^{i} x^{m}=m \xi^{i} x^{m} \\
& n_{\xi} \xi^{i} x^{m}=i \xi^{i} x^{m}
\end{aligned} \quad(i=0,1) .
$$

Scaling operators can be constructed by exponentiating the number operators. For example

$$
\begin{aligned}
& q^{n=} \xi^{i} x^{m}=q^{m} \xi^{i} x^{m}=\xi^{i}(q x)^{m} \\
& q^{n^{n}} \xi^{i} x^{m}=q^{i} \xi^{i} x^{m}=(q \xi)^{i} x^{m}
\end{aligned}
$$

and for an arbitrary function

$$
\begin{aligned}
& q^{n_{x}} F(x, \xi)=F(q x, \xi) \\
& q^{n_{\imath}} F(x, \xi)=F(x, q \xi) .
\end{aligned}
$$

By analogy with the $x$ derivatives, it is convenient to introduce the formal inverse of $\xi$ which should only be used with the number operator for $\xi$. For consistency with the q-relations it must obey

$$
\xi^{-1} x=q x \xi^{-1}
$$

Then on a general function

$$
\xi^{-1} n_{\xi}\left(F_{0}(x)+\xi F_{1}(x)\right)=\xi F_{1}(x)
$$

Now it is straightforward to read off the appropriate representation of the derivatives from their action on functions in $(4 \cdot 18,4.20)$. For the solution $r=q$

$$
\begin{aligned}
& \partial_{x}=x^{-1} q^{2 n_{\varepsilon}} \frac{q^{2 n_{x}}-1}{q^{2}-1} \\
& \partial_{\xi}=\xi^{-1} n_{\xi}
\end{aligned}
$$


and for $r=q^{-1}$

$$
\begin{aligned}
& \partial_{x}=x^{-1} \frac{q^{-2 n_{x}}-1}{q^{-2}-1} \\
& \partial_{\xi}=\xi^{-1} n_{\xi} q^{-2 n_{x}} .
\end{aligned}
$$

Note that in the limit $q \rightarrow 1$ both solutions approach the usual derivatives.

Before writing the coproduct form of the operator equations, we first note that since a monomial can have only zero or one power of $\xi$, the number operator $n_{\xi}$ can be written for arbitrary $s$ as

$$
n_{\xi}=\frac{q^{s n_{\xi}}-1}{q^{s}-1} .
$$

For the solution $r=q$ this allows us to rewrite the term in (4.19) as

$$
1+\left(q^{2}-1\right) \xi \partial_{\xi}=q^{2 n \xi} .
$$

Also, for $r=q^{-1}$, in (4.21)

$$
1+\left(q^{-2}-1\right) x \partial_{x}=q^{-2 n_{x}} .
$$

These relations allow a simpler form for the operator equations.

As mentioned earlier, the operator equations $(4.19,4.21)$ tell how the derivatives act on a pair of functions. If we regard each of the functions as lying in a vector space, then the operator equations are similar to the coproduct discussed for the algebra. We can read the coproduct from $(4.19,4.21)$, using the above relations for simplification. For the solution $r=q$ we find

$$
\begin{aligned}
& \Delta\left(\partial_{x}\right)=\partial_{x} \otimes q^{2 n_{\xi}}+q^{2 n_{x}+n_{\xi}} \otimes \partial_{x} \\
& \Delta\left(\partial_{\xi}\right)=\partial_{\xi} \otimes 1+q^{n_{\xi}} \otimes \partial_{\xi}
\end{aligned}
$$

and for $r=q^{-1}$

$$
\begin{aligned}
& \Delta\left(\partial_{x}\right)=\partial_{x} \otimes 1+q^{-2 n_{x}-n_{\xi}} \otimes \partial_{x} \\
& \Delta\left(\partial_{\xi}\right)=\partial_{\xi} \otimes q^{-2 n_{x}}+q^{-n_{x}} \otimes \partial_{\xi} .
\end{aligned}
$$

These coproducts are consistent with the derivative algebra (4.7). 


\subsection{Representation of the A.igebra}

The quantum super-plane coordinates were introduced as a vector acted on by the quantum group $\mathrm{GL}_{\mathrm{q}}(1 / 1)$. This description also leads to the action of the quantum algebra on the super-plane. Using the number operators, we will be able to write a representation of the generators similar to the representations of the derivatives.

Begin by writing the coordinate vector $V$ as a variable quantum matrix $A$ acting on a constant vector $V_{0}$ :

$$
V=A V_{0}
$$

Now apply the exterior derivative to $V$. Since $V_{0}$ is constant, $\delta V_{0}=0$ and we have

$$
\delta V=\Omega A V_{0}=\Omega V
$$

where we have used $\delta A=\Omega A$ from (3.3). This relation is invariant under a rotation of $V_{0}$ by a constant matrix $A_{1}$ :

$$
\begin{aligned}
& V=A_{0}^{\prime} V_{0}^{\prime}=\left(A_{0} A_{1}^{-1}\right)\left(A_{1} V_{0}\right) \\
& \delta V=\Omega^{\prime} V=\Omega V
\end{aligned}
$$

where we have used the fact that the Cartan-Maurer forms are invariant under right-multiplication by a constant matrix. Thus this description is independent of the particular choice of $V_{0}$.

The matrix equation (4.27) allows us to read of the action of $\delta$ on the coordinates. We find

$$
\begin{aligned}
& \delta x=\left(\omega_{X}+(1-\phi) \omega_{Y}\right) x+v_{+} \xi \\
& \delta \xi=\left(\omega_{X}-(1+\phi) \omega_{Y}\right) \xi+v_{-} x
\end{aligned}
$$

where $\phi$ is the arbitrary mixing parameter introduced in (3.11). Using the expansion of $\delta$ in (3.6), the action of the generators is seen to be

$$
\begin{array}{rlrl}
X x & =x & X \xi & =\xi \\
Y x & =(1-\phi) x & Y \xi & =-(1+\phi) \xi \\
\nabla_{+} x & =\xi & \nabla_{+} \xi & =0 \\
\nabla_{-} x & =0 & \nabla_{-} \xi & =x .
\end{array}
$$


Note that the generator $X$ acts much like the identity. This will not be true on an arbitrary monomial.

Next we wish to obtain the action of the generators on products of coordinates. This can be expressed in the form of operator equations similar to those obtained for the derivatives. Again we start with the exterior derivative and its Leibniz rule. Applying it to a product of coodinates, we can then commute the Cartan-Maurer forms to the left and read off the action of the generators. (This follows exactly the procedure used for the algebra in chapter 3.) So we must know how to commute Cartan-Maurer forms through coordinates. From (4.26) we see that the coordinates may be written

$$
\begin{aligned}
& x=a x_{0}+\beta \xi_{0} \\
& \xi=\gamma x_{0}+d \xi_{0} .
\end{aligned}
$$

Here the group parameters correspond to the matrix $A$ used to define the CartanMaurer forms by $\Omega=\delta A A^{-1}$. We know how to commute the 1 -forms through these group parameters from chapter 3 . On the other hand, the constant coordinates $x_{0}$ and $\xi_{0}$ are not related to $A$, so the 1-forms will obey the classical (graded) commutation rules with them. So we see that the Cartan-Maurer forms commute with the coordinates the same as with group parameters. These relations are the same for all group parameters, and are given in $(3.10,3.12)$. Then for the coordinates we write

$$
\begin{array}{ll}
x v_{ \pm}=q v_{ \pm} x & \xi v_{ \pm}=q v_{ \pm} \xi \\
x \omega_{X}=f \omega_{X} x & \xi \omega_{X}=-f \omega_{X} \xi \\
x \omega_{Y}=\omega_{Y} x & \xi \omega_{Y}=-\omega_{Y} \xi .
\end{array}
$$

Here we consider both solutions, $f=q$ or $q^{2}$.

Now applying $\delta$ to a coordinate, using the Leibniz rule, and commuting the 1 -forms to the left we find the operator equation for the generators acting on 
coordinates. Again using $(\cdot)$ to indicate arbitrary functions to the right, we find

$$
\begin{aligned}
X x \cdot & =x \cdot+f x X . & X \xi \cdot & =\xi \cdot+f \xi X . \\
Y x \cdot & =(1-\phi) x \cdot+x Y . & Y \xi \cdot & =-(1+\phi) \xi \cdot+\xi Y . \\
\nabla_{+} x \cdot & =\xi \cdot+q x \nabla_{+} \cdot & \nabla_{+} \xi \cdot & =-q \xi \nabla_{+} \cdot \\
\nabla_{-} x \cdot & =q x \nabla_{-} \cdot & \nabla_{-} \xi \cdot & =x \cdot-q \xi \nabla_{-} .
\end{aligned}
$$

Note that $Y$ does not have any factors of $q$ in its equation. It obeys an operator equation similar to the classical case, and we will not consider it further.

Finally, we may iterate the above equations to determine the operator relations for the generators on an arbitrary monomial in $x$ and $\xi$. Then any furction can be written as a sum of monomials. Writing an arbitrary function as $F(x, \xi)=F_{0}(x)+\xi F_{1}(x)$ like before, we find

$$
\begin{aligned}
& X F(x, \xi) \cdot=\frac{F(f x, f \xi)-F(x, \xi)}{f-1}+F(f x, f \xi) X \\
& \nabla_{+} F(x, \xi) \cdot=\xi x^{-1} \frac{F\left(q^{2} x, \xi\right)-F(x, \xi)}{q^{2}-1} \cdot+(-1)^{F} F(q x, q \xi) \nabla_{+} \cdot \\
& \nabla_{-} F(x, \xi) \cdot=x F_{1}(x) \cdot+(-1)^{F} F(q x, q \xi) \nabla_{-} \cdot
\end{aligned}
$$

As we did for the derivatives, taking the above equations with no function to the right of $F$ gives the action of the generators on $F$ alone. Then we can use the number operators to write a representation of the quantum generators. The result is

$$
\begin{aligned}
X & =\frac{f^{n_{s}+n_{\xi}}-1}{f-1} \\
\nabla_{-} & =\xi x^{-1} \frac{q^{2 n_{s}}-1}{q^{2}-1} \\
\nabla_{+} & =x \xi^{-1} n_{\xi}
\end{aligned}
$$

where $f=q$ or $q^{2}$. Note the similar form to the derivative representation in equations $(4.22,4.23)$. Finally, we could use the operator equations to determine the coproduct. This would give the same result as before. This is because both derivations use the same q-relations between 1 -forms and coordinates or group parameters. In fact, we could have started with the previously determined coproduct and applied it to monomials to determine the action of the generators. 


\section{Chapter 5}

\section{Quotient Space Geometry}

In this chapter we develop the geometry of a quotient space. Cartan-Maurer forms are constructed in the usual way. Although they are not invariant, invariant bilinear forms can be constructed on the manifold. A symmetric bilinear provides a Riemannian metric, for which we discuss the Riemannian geometry. An invariant almost complex structure is constructed, and extended to a complex structure. If the complex manifold admits a closed hermitian 2-form, it is Kähler. In this case we find a simple form for the Ricci curvature. Details of the mathematics may be found in [19] volume 2 .

\subsection{Quotient Space $G / H$}

We begin by reviewing the Riemannian geometry of a quotient space [20]. Consider a real group $G$ with subgroup $H$. For a fixed $m \in G$, the set of $g \in G$ of the form $g=m h$ as $h \in H$ runs through all of $H$ defines the left coset $g=m H$ represented by $m$. The space of all such cosets is the quotient space $\mathcal{M}=G / H$. G may be regarded as a fiber bundle over $G / H$ with fibers $H$. Decompose the algebra of $G$ by $G=M \oplus H, M \cap H=\emptyset$. We will use indices $r, s, t, \ldots$ for generators $L_{r} \in G, a, b, c, \ldots$ for $L_{a} \in M$, and $i, j, \ldots$ for $L_{i} \in H$. The generators of $G$ obey $\left[L_{r}, L_{s}\right]=L_{t} C^{t}{ }_{r s}$, and for a closed subalgebra $H \subset G, C^{a}{ }_{i j}=0$. Define the exponentials $g\left(y^{r}\right)=\exp \left(L_{r} y^{r}\right), m\left(x^{a}\right)=\exp \left(L_{a} x^{a}\right)$, and $h\left(t^{i}\right)=\exp \left(L_{i} t^{i}\right)$. 
Then any element $g\left(y^{r}\right)$ may be uniquely written as

$$
g\left(y^{r}\right)=m\left(x^{a}\right) h\left(t^{i}\right) .
$$

The parameters $x^{a}$ are coordinates for the element of $\mathcal{M}$ represented by $m\left(x^{a}\right)$, which defines a local section of the bundle by $m(x): G / H \rightarrow G$. The left action of $G$ on $\mathcal{M}$ is defined by left multiplication of $m\left(x^{a}\right)$ by an element of $G$ and refactoring the product as in (5.1):

$$
\begin{aligned}
& g_{0} m\left(x^{a}\right)=g\left(y^{\prime}\right)=m\left(x^{\prime a}\right) h\left(t^{i}\right) \\
& g_{0}: m\left(x^{a}\right) \mapsto m\left(x^{\prime a}\right)=g_{0} m\left(x^{a}\right) h^{-1}\left(t^{i}\right) .
\end{aligned}
$$

The Cartan-Maurer 1-forms on $\mathcal{M}$ are defined by

$$
m^{-1} d m=L_{r} e^{r}
$$

In components $e^{r}(x)=e^{r} a(x) d x^{a}$, where the upper index $(r)$ is a label taking values in $G$, and the lower index $(a)$ is a coordinate index taking values in $M$. The forms obey the Cartan-Maurer equations

$$
d e^{r}=-\frac{1}{2} C^{r} e^{s} e^{t} .
$$

The $e^{a}\left(L_{a} \in M\right)$ at each point $x \in \mathcal{M}$ span the cotangent space $T \mathcal{M}_{x}^{*}$. For these forms define inverse vector fields $E_{a}(x)=E^{b}{ }_{a}(x) \partial_{b}$ which span the tangent space $T \mathcal{M}_{x}$ and obey

$$
e^{a}\left(E_{b}\right)=e_{c}^{a} E_{b}^{c}=\delta_{b}^{a} \text {. }
$$

Under the left $G$-action defined in (5.2), the Cartan-Maurer forms transform as

$$
\begin{aligned}
\left(m^{\prime}\right)^{-1} d m^{\prime} & =h\left(m^{-1} d m\right) h^{-1}+h d h^{-1} \\
L_{\tau} e^{\tau} & =h L_{s} h^{-1} e^{9}+h d h^{-1} .
\end{aligned}
$$

The forms transform by an adjoint $H$ rotation. The transformed $e^{i}$ associated with generators in $H E$ also have an inhomogeneous piece from the last term in (5.6). For the $e^{a}$ associated with generators in $M$, the infinitessimal form of (5.6) is $e^{\prime a}=e^{a}+t^{i} \delta_{L,} e^{a}$ where

$$
\begin{aligned}
& \delta_{L_{1}} e^{a}=-C^{a}{ }_{i b} e^{b} \\
& \delta_{L_{1}} E_{a}=E_{b} C^{b}{ }_{i a} .
\end{aligned}
$$


where the second equation is obtained using (5.5).

Although the Cartan-Maurer forms are not left $G$-invariant, invariant bilinear forms may be constructed. Consider

$$
\begin{aligned}
& N=N_{a b} e^{a} e^{b} \\
& N(U, V)=N_{a b} e^{a}(U) e^{b}(V) \quad U, V \in \mathrm{T} \mathcal{M} .
\end{aligned}
$$

$N$ is left $G$-invariant if the coefficients $N_{a b}$ are constant on $\mathcal{M}$ and if for $\delta_{L_{i}}$ defined in (5.7) $\delta_{L_{i}} N=0$ for all $L_{i} \in H$. This is true if

$$
\begin{aligned}
& \partial_{a} N_{b c}=0 \\
& N_{a b} C^{e}{ }_{i b}+N_{e b} C^{e}{ }_{i a}=0 \quad \text { for all } L_{i} \in H .
\end{aligned}
$$

$N$ is a 2 -form if $N_{a b}=-N_{b a}$. If $N_{a b}=N_{b a}, N$ is an inner product of vectors on $\mathcal{M}$.

\subsection{Riemannian Geometry of $G / H$}

For a nondegenerate tensor $N_{a b}=N_{b a}$ obeying (5.9), we take $g_{a b} \equiv N_{c d} e_{a}^{c} e_{b}^{d}$ to be the Riemannian metric on $\mathcal{M}$ with line element

$$
\begin{aligned}
d s^{2} & =g_{a b} d x^{a} d x^{b} \\
& =N_{c d} e_{a}^{c} e^{d}{ }_{b} d x^{a} d x^{b} .
\end{aligned}
$$

The Cartan-Maurer forms may be regarded as vielbein for this metric.

A covariant derivative acts on vectors $V=V^{a} E_{a}$ as

$$
\nabla V=\left(d V^{a}+\omega^{a}{ }_{b} V^{b}\right) E_{a} .
$$

The connection form $\omega^{a}{ }_{b}$ is uniquely determined by the requirements of vanishing torsion:

$$
T^{a}=d e^{a}+\omega_{b}^{a} e^{b}=0
$$

and metric consistency:

$$
\nabla g_{a b}=0 \Rightarrow N_{a e} \omega_{b}^{e}+N_{b e} \omega_{a}^{e}=0 \text {. }
$$


For convenience define the constants

$$
D^{a}{ }_{b c}=N^{a e} C^{f}{ }_{b e} N_{f c} \text {. }
$$

Then the connection $\omega^{a}{ }_{b}=\omega^{a}{ }_{r b} e^{r}$ has components

$$
\begin{aligned}
& \omega^{a}{ }_{b b}=\frac{1}{2}\left(C^{a}{ }_{c b}-D_{c b}^{a}-D_{b c}^{a}\right) \\
& \omega^{a}{ }_{i b}=C^{a}{ }_{i b} .
\end{aligned}
$$

Finally, the curvature 2-form is defined by $\nabla \nabla V=R^{a}{ }_{b} V^{b} E_{a}$ with

$$
\begin{aligned}
R_{b}^{a} & =d \omega^{a}{ }_{b}+\omega^{a}{ }_{c} \omega^{c}{ }_{b} \\
& =\frac{1}{2} R_{c d^{a}}{ }_{b} e^{c} e^{d} .
\end{aligned}
$$

The form of the Riemann tensor $R_{c d}{ }^{a}{ }_{b}$ for $\omega^{a}{ }_{b}$ in (5.15) is complicated and we will not write it. However, as noted in the second line of (5.16) all dependence on the inhomogeneously transforming $e^{i}$ vanishes.

\subsection{Almost Complex. Structure on $G / H$}

An almost complex structure on a real manifold $\mathcal{M}$ is a tensor field $J$ which at each point $x \in \mathcal{M}$ is an automorphism of the tangent space $\mathrm{T} \mathcal{M}_{x}$ obeying $J^{2}=-1$ :

$$
\begin{gathered}
J: V \mapsto J(V) \text { for } V \in \mathrm{TM} \\
J^{2}(V)=-V .
\end{gathered}
$$

Now consider the complexified tangent space $\mathrm{T} \mathcal{M}_{\mathbf{c}}=\mathrm{T} \mathcal{M} \otimes \mathrm{C}$. Construct the projection operators

$$
P=\frac{1}{2}(1-i J) \quad \bar{P}=\frac{1}{2}(1+i J)
$$

which obey

$$
J P=i P \quad J \bar{P}=-i \bar{P} .
$$

Thus $P$ and $\bar{P}$ project onto subspaces of TMc with eigenvalues under $J$ of $+i$ and $-i$, respectively. Split $T \mathcal{M}_{\mathbf{c}}$ into subspaces $T \mathcal{M}_{\mathbf{c}}=\mathrm{T} \mathcal{M}^{(1,0)} \oplus \mathrm{T} \mathcal{M}^{(0,1)}$ by 
the mappings $P: \mathrm{T} \mathcal{M} \rightarrow \mathrm{T} \mathcal{M}^{(1,0)}$ and $\bar{P}: \mathrm{T} \mathcal{M} \rightarrow \mathrm{T} \mathcal{M}^{(0,1)}$. The complex vectors obey

$$
\begin{array}{ll}
J V^{(1,0)}=i V^{(1,0)} & V^{(1,0)} \in \mathrm{T} \mathcal{M}^{(1,0)} \\
J V^{(0,1)}=i V^{(0,1)} & V^{(0,1)} \in \mathrm{T} \mathcal{M}^{(0,1)} .
\end{array}
$$

For components, the real indices $a, b, c, \ldots$ will be replaced by the complex indices $l, m, n, \ldots$ for $(1,0)$ vectors and $\bar{l}, \bar{m}, \bar{n}, \ldots$ for $(0,1)$ vectors. For example, a vector $V=V^{a} \partial_{a}$ is complexified and split into $V^{(1,0)}=V^{m} \partial_{m}$ and $V^{(0,1)}=V^{m} \partial_{m}$, with $\overline{V^{(1,0)}}=V^{(0,1)}$. All of these constructions with $J$ on the tangent space TM carry over naturally to the cotangent space $T \mathcal{M}^{*}$. Also, we may choose a complex basis for the antihermitian generators $L_{a}$ such that $L_{m}^{\dagger}=-L_{m}$. This implies $\overline{e^{m}}=e^{m}$ and $\overline{E_{m}}=E_{m}$.

For a quotient space $\mathcal{M}=G / H, J$ can be expanded in the vectors and forms constructed on $\mathcal{M}$ as

$$
\begin{aligned}
& J=E_{a} J^{a}{ }_{b} e^{b} \\
& J^{2}=-1 \Rightarrow J^{a}{ }_{c} J_{b}^{c}=-\delta^{a}{ }_{b} .
\end{aligned}
$$

In complex coordinates, (5.19) shows that

$$
J_{n}^{m}=i \delta_{n}^{m} \quad J_{n}^{m}=-i \delta^{m}{ }_{n} .
$$

$J$ will be left $G$-invariant if the tensor $J^{a}{ }_{b}$ obeys

$$
\begin{aligned}
& \partial_{a} J^{b}{ }_{c}=0 \\
& C^{a}{ }_{i c} J^{c}{ }_{b}-J^{a}{ }_{c} C^{c}{ }_{i b}=0
\end{aligned}
$$

or in complex components

$$
C^{m}{ }_{\text {in }}=0 \quad C^{m}{ }_{\text {in }}=0 .
$$

$J$ is invariant if generators in $H$ do not mix barred and unbarred generators.

\subsection{Complex Structure on $G / H$}

As described so far, the almost complex structure $J$ is a way of splitting the real valued tangent vectors into conjugate pairs of complex valued vectors. $J$ is said 
to be integrable if it can be derived from holomorphic coordinates on a complex manifold. (On such a manifold, the transition functions between different coordinate patches are holomorphic, or complex analytic, functions of the coordinates. The manifold is said to have a complex structure.) There are several equivalent conditions for integrability of $J[19]$. Recall that $J$ was used to define the $(1,0)$ and $(0,1)$ spaces of vectors and forms with unbarred and barred components, repectively. Generalizing, define $\mathcal{A}^{(p, q)}$ to be the space of $p+q$ forms with $p(1,0)$ indices and $q(0,1)$ indices. With this separation, $J$ is integrable if

$$
\begin{array}{lll}
d \alpha \in \mathcal{A}^{(2,0)} \oplus \mathcal{A}^{(1,1)} & \text { for } & \alpha \in \mathcal{A}^{(1,0)} \\
d \alpha \in \mathcal{A}^{(0,2)} \oplus \mathcal{A}^{(1,1)} & \text { for } & \alpha \in \mathcal{A}^{(0,1)} .
\end{array}
$$

Using the Cartan-Maurer equations (5.4) this is equivalent to the the conditions on the structure constants of $G$

$$
C_{m n}^{I}=0 \quad C_{m n}^{l}=0 .
$$

Then a quotient space $\mathcal{M}=G / H$ with structure constants for $\underline{G}$ satisfying (5.24) and (5.26) is a complex manifold.

\subsection{Kählerian Geometry of $G / H$}

A bilinear form is said to be hermitian if

$$
N(J U, J V)=N(U, V)
$$

Hermitian inner products $N$ (obeying $N(U, V)=N(V, U)$ ) and 2-forms $\mathcal{K}$ (obeying $\mathcal{K}(U, V)=-\mathcal{K}(V, U))$ can be related by

$$
N(U, V)=\mathcal{K}(U, J V)
$$

A nondegenerate hermitian closed 2 -form $\mathcal{K}(d \mathcal{K}=0)$ on a complex manifold is called a Kähler form, and the manifold is a Kähler manifold [19] . Equation (5.28) defines the Kähler metric $d s^{2}=N(d x, d x)$. 
For complex $\mathcal{M}=G / H$ expand an invariant hermitian 2-form $\mathcal{K}$ (obeying (5.9)) as

$$
\begin{aligned}
\mathcal{K} & =\frac{1}{2} K_{a b} e^{a} e^{b} \\
& =K_{m \pi} e^{m} e^{n}
\end{aligned}
$$

where in the second line $K_{m n}=0=K_{m n}$ by (5.27). Using the Cartan-Maurer equations (5.4), $d \mathcal{K}=0$ if

$$
K_{a e} C_{b c}^{e}+K_{b e} C^{e}{ }_{c a}+K_{c e} C_{a b}^{e}=0 .
$$

The Kähler metric is

$$
\begin{aligned}
& N_{a b}=K_{a c} J_{b}^{c} \\
& N_{m n}=-i K_{m n} \quad N_{m n}=i K_{m n}
\end{aligned}
$$

and (5.30) relates the components $N_{m \bar{n}}=N_{\bar{n} m}$. In terms of the constants defined in (5.14) we find

$$
\begin{aligned}
& C^{m}{ }_{l n}+D^{m}{ }_{l n}-D^{m}{ }_{n d}=0 \\
& C^{m}{ }_{l n}+D^{m}{ }_{l n}+D^{m}{ }_{n l}=0 .
\end{aligned}
$$

Since the structure constants for a complex quotient space obey (5.24) and (5.26), the connection components (5.15) take the simple form

$$
\begin{aligned}
\omega_{m l}^{k}=-D_{m l}^{k} & \omega_{m l}^{k}=C_{m l}^{k} & \omega_{i l}^{k}=C^{k}{ }_{i l} \\
\omega_{m l}^{k}=-D_{m l}^{k} & \omega_{m l}^{k}=C_{m l}^{k} & \omega_{i l}^{k}=C^{k}{ }_{i l} \\
\omega^{k}{ }_{r l}=0 & \omega^{k}{ }_{r l}=0 . &
\end{aligned}
$$

Note that the connection matrix does not $\operatorname{mix}(1,0)$ and $(0,1)$ components. In what follows we will consider only the $(1,0)$ subspace, with $(0,1)$ components given by complex conjugation. Also, we note that these components are equal to the components of the difference operator $\varphi=\mathcal{L}-\nabla$ discussed in previous work $[10,11]$

We now proced to the curvature. From the definition (5.16), we find

$$
\begin{aligned}
R_{m n}{ }^{k} l= & -C_{m k^{\prime}}^{{ }^{k}} C^{k^{\prime}}{ }_{n l}-C^{k}{ }_{m i} C^{i}{ }_{n l} \\
& -D^{k}{ }_{k m} C^{k^{\prime}}{ }_{n l}+C^{k}{ }_{n k^{\prime}} D^{k^{\prime}}{ }_{l m}+C^{k^{\prime}}{ }_{m n} D^{k} l k^{\prime} .
\end{aligned}
$$


Note that the Riemann curvature is a $(1,1)$ form. In fact, given a connection preserving a hermitian metric, if the coordinates are holomorphic then the curvatur. is purely a $(1,1)$ form [21]. This is equivalent to the conditions (5.24) and (5.26) for a complex structure.

For an $\mathrm{N}$ dimensional Kähler manifold the totaily antisymmetric product

$$
\begin{aligned}
\operatorname{det}(e)=\bigwedge_{m} e^{m} & =e^{1} \wedge e^{2} \wedge \ldots \wedge e^{N} \\
& =\operatorname{det}\left(e^{m} l\right) d z^{1} d z^{2} \ldots d z^{N}
\end{aligned}
$$

spans the 1 complex dimensional space of $(\mathrm{N}, 0)$ forms. This is known as the canonical or determinant line bundle of the manifold. The covariant derivative and curvature of this form are

$$
\begin{aligned}
\nabla \operatorname{det}(e) & =\omega_{m}^{m} \operatorname{det}(e) \\
\nabla \nabla \operatorname{det}(e) & =R_{m}^{m} \operatorname{det}(e) .
\end{aligned}
$$

The 2-form $R$ is the Ricci curvature of the manifold and is the trace of the Riemann curvature. Taking the trace of (5.34) and using (5.32) we have

$$
\begin{aligned}
R_{m n} & =R_{m n}{ }^{l} l \\
& =-C_{m k}^{l}{ }_{m i} C^{k}-C^{l}{ }_{m i} C^{i}{ }_{n l}-C^{k}{ }_{m n}\left(C^{l}{ }_{k l}+C^{\top}{ }_{k l}\right) .
\end{aligned}
$$

It can be shown that this Ricci form is closed and $G$-invariant. Note that all dependence on the metric $N_{a b}=K_{a c} J^{c}{ }_{b}$ has vanished from the Ricci cuvature. This type of metric gives a unique result for the curvature.

\subsection{Discussion}

It is clear how to apply these results to a group $G$ with subgroup $H$. Decomposing the algebra as $\underline{G}=M \oplus H, M \cap H=\emptyset$, choose an almost complex structure $J$ on $M$. The choice of $J$ may be based on the generators in $M$, e.g. $L_{m}^{\dagger}=-L_{m}$. For the $(1,0)$ and $(0,1)$ decomposition based on $J$, the structure constants of $G$ must obey (5.24) for $G$-invariance of $J$ and (5.26) for $\mathcal{M}=G / H$ to be complex. A 2 -form $\mathcal{K}$ is $G$-invariant if it obeys (5.9) and closed if it obeys (5.30). If $\mathcal{K}$ is 
hermitian, $\mathcal{M}$ is Kähler and equations (5.33-37) apply yielding the connection and curvatures.

These results are easily extended to $Z_{2}$ graded super-algebras. We have chosen index ordering so that most of the results presented need no extra grading signs. The main result, the Ricci tensor, does need to be modified. The correct grading for the trace of the Riemann tensor is

$$
R_{A B}=(-1)^{C} R_{A B}^{C} C
$$

Note also that the purely fermionic components of symmetric tensors become antisymmetric, and vice versa. See the appendix for details.

For finite dimensional groups, the results are directly valid. If $G$ is infinite dimensional, contraction of indices may involve infinite sums. These sums must converge for the results to be valid. For the examples from string theory, we will see that the trace of the connection diverges but the trace of the Riemann tensor converges. 


\section{Chapter 6}

\section{String Diffeomorphism Groups}

In this chapter we apply the results of the previous chapter to the diffeomorphism groups of string theory. The main goal is to calculate the Ricci curvature of quotient spaces formed from these groups. As discussed in the introduction, this corresponds to the ghost contribution to the anomaly of the energy-momentum tensor. For the bosonic string, the group is $\operatorname{Diff}\left(\mathrm{S}^{1}\right)$. There are two choices of subgroup which admit a Kähler form. For the superstring the group is super$\operatorname{Diff}\left(S^{1}\right)$. In the Neveu-Schwarz sector their are two choices of subgroup which are Kähler. In the Ramond sector their is only one Kähler quotient space. Here we will calculate the Ricci curvature of these Kähler manifolds. Implications for string theory will be discussed in the next chapter.

\subsection{Quotient Spaces for the String}

First we apply the results of the last chapter to the symmetry groups of string theory. As discussed in [10], the open bosonic string in $D$ spacetime dimensions has as its phase space the space of loops in $R^{D}$. The reparameterizations of the circle $S^{1}$ form the group $G=\operatorname{Diff}\left(\mathrm{S}^{1}\right)$. The algebra $G=\operatorname{Diff}\left(\mathrm{S}^{1}\right)$ is based on the relation

$$
\left[f(\sigma) \frac{\partial}{\partial \sigma}, g(\sigma) \frac{\partial}{\partial \sigma}\right]=\left(f g^{\prime}-g f^{\prime}\right) \frac{\partial}{\partial \sigma}
$$


Fourier expanding gives the generators $L_{r}=e^{-i r \sigma} \frac{\partial}{\partial \sigma}$ which obey

$$
\begin{aligned}
& {\left[L_{r}, L_{s}\right]=i(r-s) L_{r+s} \quad r, s \in \mathbf{Z}} \\
& L_{r}^{\dagger}=-L_{-r}
\end{aligned}
$$

so the structure constants of $\operatorname{Diff}\left(\mathrm{S}^{1}\right)$ are

$$
C^{t}{ }_{r s}=i(r-s) \delta^{t}{ }_{r+s} \quad r, s, t \in \mathbf{Z} .
$$

The subalgebras of $G=\operatorname{Diff}\left(\mathrm{S}^{1}\right)$ are $H_{0}$ generated by $\left\{L_{0}\right\}$ and $H_{n}$ generated by $\left\{L_{0}, L_{+n}, L_{-n}\right\}$. The subgroups are $H_{0}=\mathrm{U}(1) \cong \mathrm{S}^{1}$ and $H_{n}=\mathrm{SL}(2, \mathbf{R})$.

Consider first Diff $\left(S^{1}\right) / S^{1}$ with subalgebra $\underline{H}_{0}$. The indices $r \in \mathbf{Z}$ in $(6.2,6.3)$ take values $a \neq 0$ for $M$ and $i=0$ for $H_{0}$. The choice of $J$ suggested by (6.2) is

$$
\begin{array}{ll}
J_{n}^{m}=i \delta_{n}^{m} & m, n>0 \\
J^{m}=-i \delta_{n}^{m} & \bar{m}=-m<0
\end{array}
$$

so indices take values $m>0$ and $\bar{m}=-m<0$. The structure constants (6.3) obey (5.24) ( $J$ is $G$-invariant) and (5.26) ( $J$ is integrable), so $\mathcal{M}=G / H$ is complex.

From (5.9) the 2-form $\mathcal{K}=K_{a b} e^{a} e^{b}$ is $G$-invariant if $(a+b) K_{a b}=0$. Then $\mathcal{K}$ has components

$$
K_{a b}=f(a) \delta_{a+b} \quad f(-a)=-f(a)
$$

and $\mathcal{K}$ is hermitian if $f(m)$ is real. Closure of $\mathcal{K}$ requires

$$
(a+2 b) f(a)-(b+2 a) f(b)-(a-b) f(a+b)=0
$$

which is solved by

$$
f(a)=A a^{3}+B a \quad A, B \in \mathbf{R} .
$$

Thus there is a 2-parameter class of $\operatorname{Diff}\left(S^{1}\right)$-invariant Kähler forms on $\operatorname{Diff}\left(S^{1}\right) / S^{1}$. $\mathcal{K}$ is nondegenerate if $A=0, B \neq 0$ or if $B / A \neq-n^{2}$ for all nonzero integers $n$. Note that the term proprotional to $B$ is exact, since $d e^{0}=-i \sum_{a \neq 0} a e^{a} e^{-a}$. The term proportional to $A$ is nontrivial.

Since $\mathcal{M}=\operatorname{Diff}\left(\mathrm{S}^{1}\right) / \mathrm{S}^{1}$ is complex and admits a Kähler form, the results of the previous section may apply. Care must be exercised since $\mathcal{M}$ is infinite dimensional. We will discuss this case in some detail to exhibit the convergence 
which is provided by the choice of metric. The delta function form of the structure constants gives the connection and Riemann curvature without infinite sums. From (5.33) the connection is

$$
\omega_{m}^{m}=i\left((m-2 n) \theta(1+n-m)-(2 m-n) \frac{f(n)}{f(m)} \theta(m-n)\right) \delta_{r+n}^{m}
$$

The trace $R_{m \pi}$ ' yielding the Ricci curvature is now an infinite sum which may diverge. From (5.34)

$$
\begin{aligned}
R_{m n^{l} l}=\sum_{l>0} & (-(l+m)(l-2 m) \theta(m-l+1) \\
& \left.\quad-(l+m)(2 l-m) \frac{f(m)}{f(l)} \theta(l-m)+(l+2 m)(2 l+m) \frac{f(m)}{f(l+m)}\right) .
\end{aligned}
$$

The form of the metric in (6.7) with $A \neq 0$ shows that the sum of the last two terms in (6.9) converges. Furthermore, each of these terms is logarithmically divergent. Then we may shift summation indices, and find that the sum of the last two terms vanishes. Then we are left with the final result

$$
\begin{aligned}
R_{m n} & =\sum_{l=1}^{m}(l+m)(l-2 m) \delta_{m, n} \\
& =\left(-\frac{13}{6} m^{3}+\frac{1}{6} m\right) \delta_{m, n} .
\end{aligned}
$$

The Ricci form is closed and invariant as in $(6.5,6.7)$.

Now consider the subgroups $H_{n} \cong \mathrm{SL}(2, \mathbf{R})$ generated by $\left\{L_{0}, L_{n}, L_{-n}\right\}[22]$. Indices take values $a \neq 0, \pm n$ for $M$ and $i=0, \pm n$ for $H$. It is straightforward to show that $G$-invariant closed 2 -forms on $\mathcal{M}=G / H_{n}$ take the form of (6.5) with

$$
f(a)=A\left(a^{3}-n^{2} a\right) \quad A \in \mathbf{R}
$$

i.e. they are of the form (6.7) with $B / A=-n^{2}$. However, for $n \neq 1$, the generators $L_{ \pm n} \operatorname{mix}$ barred and unbarred modes. Thus $J$ is not $G$-invariant according to (5.24). For $H_{1}$ generated by $\left\{L_{0}, L_{1}, L_{-1}\right\} J$ is $G$-invariant. Also $\mathcal{M}=G / H_{1}$ is complex since (5.26) is obeyed. Then for $n=1 \mathcal{K}$ in (6.11) is Kähler and the results of chapter 5 apply. The form of the metric again ensures convergence, and for $\mathcal{M}=\operatorname{Diff}\left(\mathrm{S}^{1}\right) / \mathrm{SL}(2, \mathrm{R})$ the Ricci curvature is

$$
R_{m n}=-\frac{13}{6}\left(m^{3}-m\right) \delta_{m, n} .
$$

Again the Ricci form is $G$-invariant and closed. 


\subsection{Quotient Spaces for the Superstring}

The symmetry group of the superstring, $G=$ super- $\operatorname{Diff}\left(\mathrm{S}^{1}\right)$, consists of the reparameterizations of the commuting coordinate on $\mathrm{S}^{1}$ and a single Grassmann coordinate in superspace. The algebra is obtained by adding to $\operatorname{Diff}\left(\mathrm{S}^{1}\right)$ a set of Grassmann-odd generators $F_{\rho}$ which obey $F_{\rho}^{\dagger}=-F_{-\rho}$. We will use Greek letters for fermionic indices, which are integer valued in the Ramond sector and halfintegers in the Neveu-Schwarz sector. The algebra is extended from (6.2) to

$$
\begin{aligned}
{\left[L_{r}, L_{s}\right] } & =i(r-s) L_{r+s} \\
{\left[L_{r}, F_{\sigma}\right] } & =i\left(\frac{1}{2} r-\sigma\right) F_{r+\sigma} \\
\left\{F_{\rho}, F_{\sigma}\right\}_{+} & =2 i L_{\rho+\sigma}
\end{aligned}
$$

with structure constants

$$
\begin{aligned}
& C_{r s}^{t}=i(r-s) \delta^{t}{ }_{r+\theta} \\
& C_{r \sigma}^{r}=i\left(\frac{1}{2} r-\sigma\right) \delta^{r+\sigma} \\
& C_{\rho \sigma}^{t}=2 i \delta^{t}{ }_{\rho+\sigma} .
\end{aligned}
$$

The subalgebras and generators are listed below.

$$
\begin{array}{ccl}
\text { Subalgebra } & \text { Sector } & \text { Generators } \\
H_{0} & \text { NS, R } & L_{0} \\
H_{n} & \text { NS, R } & L_{0}, L_{n}, L_{-n} \\
H_{\nu} & \text { NS } & L_{0}, L_{n}, L_{-n}, F_{\nu}, F_{-\nu} \\
H_{\tilde{0}} & \mathrm{R} & L_{0}, F_{0}
\end{array}
$$

The subgroups are $H_{0} \cong \mathrm{U}(1) \cong \mathrm{S}^{1}, H_{n} \cong \mathrm{SL}(2, \mathrm{R})$, and $H_{\nu} \cong \mathrm{OSp}(1 \mid 2)$. The group $H_{\tilde{0}}$ is sometimes referred to as $s-\mathrm{S}^{1}$.

In the NS sector $G$-invariant 2-forms on $G / H_{0}$ are

$$
\mathcal{K}=\frac{1}{2} K_{a b} e^{a} e^{b}+\frac{1}{2} K_{\alpha \beta} e^{\alpha} e^{\beta}
$$

with components

$$
\begin{array}{ll}
K_{a b}=f(a) \delta_{a+b} & f(a)=A a^{3}+B a \\
K_{\alpha \beta}=g(\alpha) \delta_{\alpha+\beta} & g(\alpha)=4 A \alpha^{2}+B
\end{array} \quad A, B \in \mathbf{R} .
$$


Again the term proportional to $B$ is exact, and $\mathcal{X}$ is nondegenerate on $G / H_{0}$ if $A=0, B \neq 0$ or if $B / A \neq-n^{2}$ for all nonzero integers $n$. For $G / H_{n}, B / A=-n^{2}$, and for $G / H_{\nu}, B / A=-4 \nu^{2}$. In the $\mathrm{R}$ sector we have $G$-invariant 2 -forms on $G / H_{\tilde{0}}$

$$
\mathcal{K}=\frac{1}{2} K_{\alpha b} e^{a} e^{b}+\frac{1}{2} K_{\alpha \beta} e^{\alpha} e^{\beta}+\frac{1}{2} K_{\alpha \beta} e^{a} e^{\beta} .
$$

The components $K_{a b}$ and $K_{\alpha \beta}$ have the same form as in (6.15) with $B=0$, and the mixed term is

$$
K_{a \beta}=h(a) \delta_{a+\beta} \quad h(a)=\Gamma a .
$$

The term proportional to the Grassmann constant $\Gamma$ is exact, since the exterior derivative of $e^{\tilde{0}}$ is $d e^{\tilde{0}}=-3 i \sum_{\alpha=\alpha \neq 0} e^{a} e^{-\alpha}$.

For a complex quotient space $G / H$, the subalgebra $H$ must contain all the real generators. In the Ramond sector this means the only possible choice is $H=H_{\overline{0}}$ generated by $L_{0}$ and $F_{0}$. Again the property $F_{\rho}^{\dagger}=-F_{-\rho}$ suggests taking positive modes for the $(1,0)$ subspace and negative modes for $(0,1)$. Then the only quotient spaces with invariant $J$ have subgroups $H_{0}$ and $H_{\frac{1}{2}}$ in the NS sector and $H_{0}$ in the $\mathrm{R}$ sector. Again the algebra ensures that $J$ is integrable, so these quotient space are Kähler. Following the procedure for the bosonic case, we see that the form of $f(a)$ and $g(\alpha)$ in (6.15) leads to a convergent trace of the Riemann tensor for $A \neq 0$. Note that the super-trace of a matrix $R$ is $R_{a}^{a}-R_{\alpha}^{\alpha}[16,23]$. Then the Ricci curvatures are

$$
\begin{aligned}
\text { NS sector, } H=S^{1}: \quad R_{m n} & =\left(-\frac{5}{4} n^{3}+\frac{1}{4} m\right) \delta_{m, n} \\
R_{\mu \nu} & =\left(-5 \mu^{2}+\frac{1}{4}\right) \delta_{\mu, \nu} \\
\text { NS sector, } H=\operatorname{OSp}(1 \mid 2): \quad R_{m n} & =\left(-\frac{5}{4} m^{3}+\frac{5}{4} m\right) \delta_{m, n} \\
R_{\mu \nu} & =\left(-5 \mu^{2}+\frac{5}{4}\right) \delta_{\mu, \nu} \\
\text { R sector, } H=s-S^{1}: \quad R_{m \pi} & =\left(-\frac{3}{4} m^{3}\right) \delta_{m, n} \\
R_{\mu \nu} & =\left(-5 \mu^{2}\right) \delta_{\mu, \nu} .
\end{aligned}
$$

\subsection{Graded Algebras}

The algebras for string diffeomorphisms are examples of Z-graded algebras. A graded algebra $G$ may be split into submodules $G_{\text {r }}$ of finite dimension obeying

$$
\left[\underline{G}_{r}, G_{\mathbf{n}}\right] \subset \underline{G}_{r+0} \quad r, s \in \mathbf{Z} .
$$


(The grading includes half-integers in the NS sector.) $G_{0}$ is a closed subalgebra so we may consider the quotient space $G / G_{0}$. The relation $G_{r}^{\dagger}=G_{-r}$ suggests an almost complex structure separating positive and negative graded submodules. The form of the structure constants $C^{r}$ st $\propto \delta_{s+t}^{r}$ obeys the conditions (5.24) and (5.26) so $G / G_{0}$ is complex. If a $G$-invariant Kähler form can be constructed, the expressions for the connection (5.33) and Riemann tensor (5.34) are valid and $\omega^{a}{ }_{b} \propto \delta_{b+r}^{a} e^{r}$ and $R_{b}^{a} \propto \delta_{b+m-n}^{a} e^{m} e^{n}$. But the Ricci tensor requires an infinite trace which may be convergent for some class of Kähler metrics. If the trace converges the Ricci form is given by

$$
R_{m n}=-\left(\sum_{l=1}^{m} C_{m, l-m}^{l} C_{-m, l}^{l-m}\right) \delta_{m, n} .
$$

(The last term in (5.37) never contributes for $\mathcal{M}=G / G_{0}$.) Here an index $r$ takes all values of the generators spanning $G_{r}$. The NS sector is simply an extension of the bosonic case with half-integer graded submodules. But in the $\mathrm{R}$ sector there is a bosonic and fermionic component of each integer graded submodule. 


\section{Chapter 7}

\section{Associated Vector Bundles}

In this section we extend the results for Kähler quotient spaces to more general vector bundles over these manifolds. The new vector bundles are extensions or restrictions of the tangent space of the marifold. Requiring the bundle to be holomorphic constrains the choice of vector space for the fiber. There is a natural choice of fiber for quotient spaces based on a graded algebra. These results are applied to the string and superstring. Implications for the string vacuum state are discussed.

\subsection{Holomorphic Vector Bundles}

In the preceeding sections we have considered the Riemannian and Kählerian geometry of the quotient space $\mathcal{M}=G / H$. These are special cases of vector bundles over $\mathcal{M}$. In the Riemannian case the fiber at $x$ is the tangent space $T \mathcal{M}_{x}$. For $\mathcal{M}$ Kähier the fiber is the $(1,0)$ subspace of the complexified tangent space $T \mathcal{M}_{x}^{c}=T \mathcal{M}_{x}^{(1,0)} \oplus T \mathcal{M}_{x}^{(0,1)}$. The Kähler metric is a hermitian inner product on $T \mathcal{M}_{x}^{(1,0)}$, and the connection acts within the $(1,0)$ subspace. In the case the vector bundle is holomorphic, i.e. the fiber transition functions between coordinate patches are holomorphic functions of the coordiantes.

For graded algebras we can consider related vector bundles over $\mathcal{M}=G / G_{0}$. The fibers are spanned by subsets of vector fields $E_{r}$ or the Cartan-Maurer forms 
$e^{r}$. Define the vector space $\mathrm{T} \mathcal{H}_{x}$ spanned by $E_{i}(x)$ for generators $L_{i} \in \mathcal{G}_{0}=H$. Decompose the total space as $V_{x} \oplus V_{x}^{\perp}=\mathrm{T} \mathcal{M}_{x}^{\mathcal{C}} \oplus \mathrm{T} \mathcal{H}_{x}$ and assign indices

$$
\begin{aligned}
& E_{m}(x) \in \mathrm{TM}_{x}^{(1,0)} \quad E_{m \hbar}(x) \in \mathrm{T} \mathcal{M}_{z}^{(0,1)} \quad E_{i}(x) \in \mathrm{T} \mathcal{H}_{x} \\
& E_{a}(x) \in V_{x} \quad E_{a^{\prime}}(x) \in V_{x}^{\perp}
\end{aligned}
$$

where unprimed indices $a$ are in $V$ and primed indices $a^{\prime}$ are in $V^{\perp}$. As before $G$ invariance requires adjoint $G_{0}$ invariance. Then this decomposition is $G$-invariant if modes in $V$ and $V^{\perp}$ are not mixed by $G_{0}$. For a graded algebra this requires that each submodule $G_{r}$ is entirely in either $V$ or $V^{\perp}$.

In the Kähler case $V_{x}=T \mathcal{M}_{x}^{(1,0)}$ and $V_{x}^{\perp}=\mathrm{T} \mathcal{M}^{(0,1)} \oplus \mathrm{T} \mathcal{H}_{x}$. We will treat the general case as a finite extension or restriction of the Kähler case. A hermitian inner product on $T \mathcal{M}^{\mathbf{c}}$ is defined by the Kähler metric. If $T_{x} \subset V_{x}$ the inner product must be extended by including a nondegenerate $N_{i j}$ obeying $N_{i l} C^{l}{ }_{k j}+N_{j l} C^{l}{ }_{k i}$ (where all indices $i, j, k, l$ are in $G_{0}$.) Also set $N_{i m}=0$ and $N_{i m}=0$. Then the hermitian inner product on $V$

$$
N\left(E_{a}, \overline{E_{b}}\right)=N_{a b}
$$

is preserved by the connection

$$
\omega^{a}{ }_{b}=-D^{a}{ }_{m b} e^{m}+C^{a}{ }_{m b} e^{m}+C^{a}{ }_{i b} e^{i}
$$

where $D^{a}{ }_{m b}=N^{a \varepsilon} C^{f}{ }_{m e} N_{f b}$. This is just an extension or restriction of the Kähler connection that operates within the space $V$ spanned by the vector fields $E_{a}$.

The curvature of the vector bundle is defined by $R^{a}{ }_{b}=d \omega^{a}{ }_{b}+\omega^{a}{ }_{c} \omega^{c}{ }_{b}$. For a holomorphic vector bundle with connertion preserving a hermitian inner product, the curvature of the connection must be a $(1,1)$ form on the base manifold. The converse is also true [21]. Thus, if we choose a bundle and connection such that the curvature is a $(1,1)$ form, the bundle is holomorphic. For the connection (7.3) the curvature is a $(1,1)$ form on $G / H_{0}$ if

$$
C_{m b}^{a^{\prime}}=0 \quad C_{m b}^{a}=0 .
$$

Using the form of the structure constants for a graded algebra, this is true if we decompose

$$
\begin{array}{ll}
E_{a} \in V & a>r_{0} \\
E_{a^{\prime}} \in V^{\perp} & a^{\prime} \leq r_{0} .
\end{array}
$$


The Kähler case is $r_{0}=0$ with $V=\mathrm{T} \mathcal{M}^{(1,0)}$. Values of $r_{0}>0$ are finite restrictions and $r_{0}<0$ are finite extentions of this case.

The Ricci form $R=R_{a}^{a}$ is the curvature of the determinant line bundle constructed as the exterior product of all forms spanning $V^{*}$, the dual space of $V$. For the graded case this is the semiinfinite form

$$
\operatorname{det}(e)=\bigwedge_{a>r_{0}} e^{a}=e^{r_{0}+1} \wedge e^{r_{0}+2} \wedge e^{r_{0}+3} \ldots
$$

including all $e^{a}$ at levels higher than $r_{0}$. The cohomolugy of such forms has been studied in reference [24]. Again the form of the metric can ensure convergence of the trace. For a finite change in connection $\Delta \omega^{a}{ }_{b}$ from the Kahler connection, the Ricci form is

$$
R=R_{K}+d \Delta \omega^{a}
$$

where $R_{K}$ is the Ricci form of Kähler geometry. For a graded algebra each vector $E_{a}$ in $V$ contributes the exact term $-C^{i}{ }_{m \pi} C^{a}{ }_{i a} e^{m} e^{\pi}$ to the Ricci form. Adding or subtracting these terms from the Kählerian Ricci form (5.37), the Ricci curvature for any $r_{0}$ is

$$
\begin{aligned}
R_{m n} & =-C^{a}{ }_{m b} C^{b^{\prime}} \quad a \quad a>r_{0}, b^{\prime} \leq r_{0} \\
& =-\sum_{a=r_{0}+1}^{r_{0}+m} C^{a}{ }_{m, a-m} C^{a-m}{ }_{-m, a} .
\end{aligned}
$$

For $G=\operatorname{Dil}^{\mp}\left(\mathrm{S}^{1}\right), H=\mathrm{S}^{1}$, the line bundle $\operatorname{det}_{r_{0}}(e)$ of forms $e^{a}, a>r_{0}$ has curvature

$$
R_{m, n}=\left(-\frac{13}{6} m^{3}+\left(r_{0}\left(r_{0}+1\right)+\frac{1}{6}\right) m\right) \delta_{m, n} .
$$

For the superstring case with $H=G_{0}$, the determinant must include fermionic modes $e^{\alpha}, \alpha>\rho_{0}$, where $\rho_{0}=r_{0}+\frac{1}{2} \delta_{0}, \delta_{0}= \pm 1(0)$ in the NS(R) sector. The curvature in the NS sector is

$$
\begin{aligned}
& R_{m n}=\left(-\frac{3}{4} m^{3}+\left(\delta_{0}\left(r_{0}+\frac{1}{2}\right)-\frac{1}{4}\right) m\right) \delta_{m, n} \\
& R_{\mu \nu}=\left(-5 \mu^{2}+\left(\delta_{0}\left(r_{0}+\frac{1}{2}\right)-\frac{1}{4}\right)\right) \delta_{\mu, \nu} .
\end{aligned}
$$

The curvature in the Ramond sector does not change from the Kähler case, since the bosonic and fermionic contributions at each integer level cancel. 


\subsection{Implications for String Theory}

It is known from the study of gauge theories that the ghosts may be identified with the Cartan-Maurer forms of the gauge group. The ghost vacuum of string theory filled to level $r_{0}+1$ is the semiinfinite product

$$
\begin{aligned}
|0\rangle_{r_{0}} & =\prod_{a>r_{0}} c^{a}=c^{r_{0}+1} c^{r_{0}+2} c^{r_{0}+3} \ldots \\
c^{a}|0\rangle_{r_{0}} & =0 \quad a>r_{0}
\end{aligned}
$$

Identifying the ghost modes with the Cartan-Maurer forms, we see that this is the semiinfinite form (7.6). The ghost contribution to the energy-momentum tensor anomaly in these vacuua has been calculated [25] and agrees with our results $(7.9,7.10)$ and with reference [12] and standard results in string theory [26].

The total string vacuum is the product of matter fields $X^{\mu}(\sigma)$ vacuua and ghost vacuum: $|0\rangle=|0\rangle_{X} \otimes|0\rangle_{r_{0}}$. The contribution of the $X^{\mu}$ vacuua to the anomaly has been calculated as the curvature of a holomorphic line bundle over (super-)Diff $\left(\mathrm{S}^{1}\right) / \mathrm{S}^{1}[10,12]$. For the bosonic string in $D$ spacetime dimensions this curvature is

$$
F_{m n}=\left(\frac{D}{12}\left(m^{3}-m\right)-2 a_{0} m\right) \delta_{m, n}
$$

where $a_{0}$ is a normal ordering constant for $L_{0}$, the generator of rigid $S^{1}$ rotations. The total curvature of the vacuum bundle is the sum of the string and ghost contributions. Reparameterization invariance of the vacum requires that the curvature vanish. Rewrite (7.9) in the suggestive form

$$
R_{m n}=\left(-\frac{13}{6}\left(m^{3}-m\right)+\left(r_{0}+2\right)\left(r_{0}-1\right) m\right) \delta_{m, n} .
$$

The nontrivial terms in the curvatures fix $D=26$. The exact terms fix the normal ordering constant to

$$
a_{0}=\frac{1}{2}\left(r_{0}+2\right)\left(r_{0}-1\right) .
$$

$a_{0}=-1$ for $r_{0}=0$, the vacuum corresponding to the canonical line bundle of $\operatorname{Diff}\left(\mathrm{S}^{1}\right) / \mathrm{S}^{1} . a_{0}$ vanishes for $r_{0}=-2,1$. This corresponds to the vacuua which just include or exclude modes for the generators of $\operatorname{SL}(2, \mathbf{R})$. These are the $\operatorname{SL}(2, \mathbf{R})$ invariant vacuua. Note that in these cases the curvature agrees with the Ricci curvature of $\operatorname{Diff}\left(S^{1}\right) / S L(2, R)$ from (6.12). 
In the Neveu-Schwarz sector of the superstring the curvature of the matter vacuum bundle is

$$
\begin{aligned}
& F_{m \bar{n}}=\left(\frac{D}{8}\left(m^{3}-m\right)-2 a_{0} m\right) \delta_{m, n} \\
& F_{\mu \nu}=\left(\frac{D}{2}\left(\mu^{2}-\frac{1}{4}\right)-2 a_{0}\right) \delta_{\mu, \nu .}
\end{aligned}
$$

Rewrite (7.10) in the similar form

$$
\begin{aligned}
& R_{m n}=\left(-\frac{5}{4}\left(m^{3}-m\right)+\left(\delta_{0}\left(r_{0}+\frac{1}{2}\right)-\frac{3}{2}\right) m\right) \delta_{m, n} \\
& R_{\mu \nu}=\left(-5\left(\mu^{2}-\frac{1}{4}\right)+\left(\delta_{0}\left(r_{0}+\frac{1}{2}\right)-\frac{3}{2}\right)\right) \delta_{\mu, \nu}
\end{aligned}
$$

The notrivial terms fix $D=10$. The exact terms fix

$$
a_{0}=\frac{1}{2}\left(\delta_{0}\left(r_{0}+\frac{1}{2}\right)-\frac{3}{2}\right) .
$$

$a_{0}=-\frac{1}{2}$ for $r_{0}=0$ and $\delta_{0}=+1$, which corresponds to the canonical line bundle of super-Diff( $\left(\mathrm{S}^{1}\right) / \mathrm{S}^{1} . a_{0}$ vanishes for $r_{0}=1, \delta_{0}=+1$ and for $r_{0}=-2, \delta_{0}=-1$. This corresponds to the vacuua which just include or exclude the modes for the generators of $\operatorname{OSp}(1 \mid 2)$. Here the curvature also agrees with the Ricci curvature of super-Diff( $\left(\mathrm{S}^{1}\right) / \mathrm{OSp}(1 \mid 2)$ from (6.17).

Finally, in the Ramond sector the curvature of the matter vacuum bundle is

$$
\begin{aligned}
& F_{m n}=\left(\frac{D}{8} m^{3}-2 a_{0} m\right) \delta_{m, n} \\
& F_{\mu \nu}=\left(\frac{D}{2} \mu^{2}-2 a_{0}\right) \delta_{\mu, \nu .} .
\end{aligned}
$$

The curvature of the ghost vacuum bundle is always

$$
\begin{aligned}
& R_{m n}=\left(-\frac{5}{4} m^{3}\right) \delta_{m, n} \\
& R_{\mu \nu}=\left(-5 \mu^{2}\right) \delta_{\mu, \nu}
\end{aligned}
$$

with no dependence on the sea level. Thus $a_{0}=0$ for all vacuua in the Ramond sector. 


\section{Appendix A}

\section{Super-Tensor Index Conventions}

In this appendix we discuss $Z_{2}$ grading sign conventions for super-tensors. Superspace is based on a Grassmann algebra. A set of Grassmann generators $\xi^{i}$ obey

$$
\xi^{i} \xi^{j}=-\xi^{j} \xi^{i} \quad\left(\xi^{i}\right)^{2}=0 .
$$

For most applications in physics the number of Grassmann generators is infinite. By extending the usual pioperties cf tensors to functions including the Grassmann generators one define tensors on superspace. Due to the sign in (1.1) signs appear in many tensor relations. Here we will derive appropriate conventions to determine the signs. Our results are similar to those of [23]. When referring to c-number quantities, we use the terms commuting, Grassmann even, and bosonic interchangeably. For quantities obeying (1.1) we use the terms anticommuting, Grassmann odd, and fermionic interchangeably.

\section{A.1 Super-Functions and Derivatives}

The $Z_{2}$ grading for vectors can be inferred from the properties of functions of Grassmann variables and their derivatives. Consider functions of two variables, $x$ and $\xi$, which are commuting and anticommuting, respectively. Since Grassmann variables are nilpotent, an expansion of such a function in powers of $\xi$ will have only two terms:

$$
F(x, \xi)=F_{0}(x)+\xi F_{1}(x) .
$$


Take first a function $f$ which is overall commuting. Then the expansion is

$$
f(x, \xi)=a(x)+\xi \beta(x)=a(x)-\beta(x) \xi
$$

where the coefficient function $a(x)$ is commuting and $\beta(x)$ is anticommuting. Now consider the left and right derivatives of $f$ with respect to $x$ and $\xi$. They are

$$
\begin{array}{ll}
\overrightarrow{\partial_{x}} f(x, \xi)=a^{\prime}(x)+\xi \beta^{\prime}(x) & f(x, \xi) \overleftarrow{\partial_{x}}=a^{\prime}(x)+\xi \beta^{\prime}(x) \\
\overrightarrow{\partial_{\xi}} f(x, \xi)=\beta(x) & f(x, \xi) \overleftarrow{\partial_{\xi}}=-\beta(x)
\end{array}
$$

We see that left and right $x$ derivatives are equal, but the left and right $\xi$ derivatives have opposite sign. Next consider a Grassmann valued function $\phi(x, \xi)$ with expansion

$$
\phi(x, \xi)=\alpha(x)+\xi b(x)=\alpha(x)+b(x) \xi .
$$

Here $\alpha(x)$ is anticommuting and $b(x)$ is commuting. Then the left and right derivatives are

$$
\begin{array}{ll}
\overrightarrow{\partial_{x}} \phi(x, \xi)=\alpha^{\prime}(x)+\xi b^{\prime}(x) & \phi(x, \xi) \overleftarrow{\partial_{x}}=\alpha^{\prime}(x)+\xi b^{\prime}(x) \\
\overrightarrow{\partial_{\xi}} \phi(x, \xi)=b(x) & \phi(x, \xi) \overleftarrow{\partial_{\xi}}=b(x)
\end{array}
$$

In this case we see that left and right derivatives are equal for both $x$ and $\xi$.

\section{A.2 Super-Vectors}

These results can be used to define index grading for super-vectors with upper or lower indices. As is conventional, we will take derivatives to have lower indices. Then the derivative of a function is a vector with a lower index, e.g. $U_{A}$. Since coordinates have upper indices and are functions on the vector space, we will take coordinates to be upper index vectors, e.g. $V^{A}$. Next we recall a result of DeWitt which states that any super-vector space may be chosen to have a pure basis, that is, one in which the basis vectors are either purely commuting or purely anticommuting. Then the Grassmann parity of a vector component is determined by its index. For $Z_{2}$ grading signs, we use the common notation based on the vector index $A$ :

$$
(-1)^{A}= \begin{cases}+1 & A \text { bosonic index } \\ -1 & A \text { fermionic index }\end{cases}
$$


This notation means that in the exponent of $(-1)$, replace the index by 0 if the index is bosonic and 1 if the index is fermionic. When considering more than one vector, grading signs depending on several indices may occur. Using 0 or 1 for indices in exponents of $(-1)$, we have for example

$$
(-1)^{A B}= \begin{cases}-1 & A \text { and } B \text { both fermionic } \\ +1 & \text { otherwise }\end{cases}
$$

Finally, an object may have an intrinsic Grassmann parity in addition to any index grading. For example, the above functions $f$ and $\phi$ are bosonic and fermionic, respectively. Then the grading notation is the same as for indices, writing the name of the object instead of the index in the exponent of $(-1)$. The name is then replaced by 0 or 1 if the object is bosonic or fermionic, respectively. For example, for a function $F$ :

$$
(-1)^{F}= \begin{cases}+1 & F \text { bosonic } \\ -1 & F \text { fermionic }\end{cases}
$$

Most of the super-tensors we will consider are extensions of the purely bosonic case, and as such have even intrinsic Grassmann parity. For the rest of this discussion we will assume that tensors have even intrinsic parity unless indicated otherwise.

The results above indicate that left and right derivatives may have different signs. Thus, we will have to distinguish between vectors with left and right indices. The index location indicates how a vector transforms under a change of basis. Left index vectors are multiplied by a rotation matrix on the left, and similarly for right indices. Covariance under such rotations will determine $Z_{2}$ grading signs associated with a particular quantity.

First consider lower index vectcrs, which can be related to the derivative of a bosonic valued function. (The derivative of a Grassmann valued function will yield a vector with odd intrinsic parity.) For a function $f$ of coordinates $x^{A}$ define a vector $U$ by

$$
U_{A}=f \frac{\overleftarrow{\partial}}{\partial x^{A}} \quad{ }_{A} U=\frac{\vec{\partial}}{\partial x^{A}} .
$$

Then from (1.1) we see that

$$
U_{A}=(-1)^{A}{ }_{A} U
$$

For fermionic components of lower-index vectors, the left- and right-index components have oppposite signg. 
To proceed, we will have to consider the transformation properties of vectors. Suppose we change coordinates by $x^{A} \rightarrow x^{\prime A}\left(x^{B}\right)$. Define left and right transformation matrices by the derivatives

$$
{ }_{A} L^{B}=\frac{\vec{\partial}}{\partial x^{A}} x^{B} \quad{ }^{B} R_{A}=x^{B} \frac{\grave{\partial}}{\partial x^{A}} .
$$

For example, these relate the derivatives

$$
\frac{\vec{\partial}}{\partial x^{A}}={ }_{A} L^{B} \frac{\vec{\partial}}{\partial x^{\prime B}} \quad \frac{\overleftarrow{\partial}}{\partial x^{A}}={\frac{\overleftarrow{\partial}}{\partial x^{B}}}^{B} R_{A} .
$$

From the above results for derivatives of functions $(1.2,1.3)$, we see that the left and right matrices are related by

$$
{ }^{A} R_{B}=(-1)^{B(A+1)}{ }_{B} L^{A} .
$$

For the rest of this discussion, difieren index orderings of a given tensor will be referred to by the same name. Thus, $R$ and $L$ are both the same rotation matrix $M$, and

$$
{ }^{A} M_{B}=(-1)^{B(A+1)}{ }_{B} M^{A}
$$

is the grading for different index orderings. Also, note from the definition (1.4) that the Grassmann parity of ${ }^{A} M_{B}$ is $(-1)^{A+B}$.

Now we can determine all grading conventions for vectors. For a rotation matrix $M$, we will also need the inverse rotation $N$ which obeys

$$
{ }^{A} M_{B}{ }^{B} N_{C}={ }^{A} \delta_{C} \quad{ }^{A} \delta_{C}= \begin{cases}1 & A=C \\ 0 & \text { otherwise } .\end{cases}
$$

The different index orderings for $N$ are related the same as $M$. Define left- and right- , upper- and lower-index vectors to have transformation rules

$$
\begin{array}{ll}
V^{A}=V^{B}{ }_{B} M^{A} & U_{A}^{\prime}=U_{B}{ }^{B} N_{A} \\
{ }^{A} V^{\prime}={ }^{A} M_{B}{ }^{B} V & { }_{A} U^{\prime}={ }_{A} N^{B}{ }_{B} U
\end{array}
$$

Then using (1.5) to rearrange indices on $M$ and using the Grassmann parity of the vectors and $M$, one can relate the left- and right-index vectors. For upper-index $V:$

$$
\begin{aligned}
{ }^{A} V^{\prime} & ={ }^{A} M_{B}{ }^{B} V \\
& =(-1)^{B(A+1)}{ }_{B} M^{A}{ }^{B} V \\
& ={ }^{B} V_{B} M^{A} .
\end{aligned}
$$


Comparing with the defined transformation properties (1.6) we see that ${ }^{A} V$ and $V^{A}$ transform the same way Thus they are equal up to a multiplicative constant. Choosing the constant to be one we inay identify

$$
{ }^{A} V=V^{A} .
$$

For lower-index $U$ :

$$
\begin{aligned}
U_{A}^{\prime} & =U_{B}{ }^{B} N_{A} \\
& =(-1)^{A(B+1)} U_{B} N^{B} \\
& =(-1)^{A+B}{ }_{A} N^{B} U_{B} \\
(-1)^{A} U_{A}^{\prime} & ={ }_{A} N^{B}(-1)^{B} U_{B} .
\end{aligned}
$$

Thus we see that $(-1)^{A} U_{A}$ transforms like ${ }_{A} U$ and we identify

$$
{ }_{A} U=(-1)^{A} U_{A} .
$$

Again we have set an arbitrary constant to one. This agrees with our earlier conclusion for lower-index vectors defined by derivatives.

\section{A.3 Super-Tensors}

Tensors are constructed simply as the product of vectors. As an example, consider the case of a tensor with one upper and two lower indices constructed from an upper-index vector $V$ and lower-index vectors $U$ and $W$. Then define the tensor $T$ by

$$
T^{A}{ }_{B C}=V^{A} U_{B} W_{C} .
$$

Different index orderings are obtained by permuting the defining vectors and including appropriate grading signs. For example, some permutations of $T$ are

$$
\begin{aligned}
T_{B C}^{A} & =(-1)^{A B} T_{B}^{A}{ }_{C} \\
& =(-1)^{A(B+C)} T_{B C}{ }^{A} \\
& =(-1)^{B C} \tilde{T}_{C B}^{A} .
\end{aligned}
$$

Note that the tensor in the last line is different from the initial one, and is defined by $T \propto V W U$, with the vertors ordered differently from the first cuse. Finally, 
indices may be changed between left and right using (1.7) for upper indices and (1.8) for lower indices.

The transformation rules for tensors are easily obtained from those for the defining vectors in (1.6). For the above example, with rotation matrix $M$ and inverse $N$,

$$
\begin{aligned}
T^{\prime A}{ }_{B C} & =V^{\prime A} U_{B}^{\prime} W_{C}^{\prime} \\
& =V^{D}{ }_{D} M^{A} U_{E}{ }^{E} N_{B} W_{F}{ }^{F} N_{C} \\
& =(-1)^{E(A+D)+F(A+B+D+E)} V^{D} U_{E} W_{F D} M^{A}{ }^{E} N_{B}{ }^{F} N_{C} \\
& =(-1)^{E(A+D)+F(A+B+D+E)} T^{A}{ }_{B C D} M^{A}{ }^{E} N_{B}{ }^{F} N_{C} .
\end{aligned}
$$

In general, a tensor transforms by the product of the transformation matrices for each of its indices, with grading signs included for permuting saturated indices until they are adjacent.

Consider now a tensor with one upper- and one lower-index defined by

$$
T^{A}{ }_{B}=V^{A} U_{B} .
$$

We wish to construct a scalar $S$ by saturating the indices. Grading signs will be needed to insure that the scalar is invariant, i.e. $S^{\prime}=S$. From the defintion of the roatation matrix $M$ and inverse $N$, we see that the quantity $V_{A}^{A} U$ is invariant:

$$
V^{\prime A}{ }_{A} U^{\prime}=V^{C}{ }_{C} M^{A}{ }_{A} N^{D}{ }_{D} U=V^{C}{ }_{C} \delta^{D}{ }_{D} U=V^{C}{ }_{C} U .
$$

But from (1.8) ${ }_{A} U=(-1)^{A} U_{A}$, so the scalar quantity is

$$
S=(-1)^{A} V^{A} U_{A}=(-1)^{A} T_{A}^{A} .
$$

This determines the appropriate grading for saturating indices on a tensor $T^{A}{ }_{B}$. It agrees with the well known form of the super-trace. Saturation of indices with other orderings, or left- or right-indices, is determined using the appropriate grading for permuting the indices. For example

$$
\begin{aligned}
S & =(-1)^{A} T^{A}{ }_{A}=T_{A}{ }^{A} \\
& =(-1)^{A}{ }^{A} T_{A}=(-1)^{A}{ }_{A} T^{A} \\
& ={ }^{A}{ }_{A} T=(-1)^{A}{ }_{A}{ }^{A} T
\end{aligned}
$$


are the ways to saturate all possible index arrangements of the tensor $T$ considered above. Finally, pairs of indices on higher rank tensors may be saturated to create a tensor with rank two lower. Grading signs are inserted to permute the indices until the saturated indices are adjacent. Then the above rules are used to sature the pair. For example,

$$
S_{B C}=(-1)^{A(1+B+C)} T_{B C A}^{A}
$$

is the correct way to form a tensor $S_{B C}$ by saturating the first and last indices of a tensor $T_{B C D \text {. }}^{A}$

Finally, we note that tensors may be multiplied by saturating indices, forming a new tensor with the remaining indices. The grading rules are easily determined using the definition of a tensor (1.9) and the grading rules (1.10). The simplest case is saturating the last index of the first tensor with the first index of the last. For example

$$
R_{B}^{A}=S_{C}^{A} T_{B}^{C}
$$

forms a new rank-2 tensor $R$ from rank-2 tensors $S$ and $T$. When transformed, the rotation matrices for the saturated indices cancel, as in the case for the scalar quantity (1.10). Other index orderings are obtained by including grading signs to permute indices until the saturated indices are adjacent. For example,

$$
R_{B}^{A}=(-1)^{A C} S_{C}^{A} T_{B}^{C}
$$

forms $R$ from $S$ and $T$, with $S$ now having index ordering $S_{A}{ }^{B}$.

The tensor multiplication rules are particularly useful when considering the tensor product of matrices. The tensor product of two matrices $R$ and $S$ can be written as a rank-4 tensor with index ordering given by

$$
(R \otimes S)^{A_{1}}{ }_{B_{1}}^{A_{2}}{ }_{B_{2}}=R^{A_{1}}{ }_{B_{1}} S^{A_{2}} B_{2}
$$

The index subscripts indicate which vector space in the tensor product the index belongs to. Then multiplication of two such matrices will require grading signs from permuting the saturated indices until they are adjacent. As seen above, no grading signs are needed in the multiplication if the indices are already adjacent. Thus, when dealing with large matrices, it is convenient to arrange the indices in 
the tensor product so that this is the case. For a tensor product, this means that the first and last indices should belong to the same vector space, the second and second to last indices belong to the same vector space, and so forth for higher tensor products. For the above example, this appropriate arrangement is

$$
(R \otimes S)^{A_{1} A_{2}}{ }_{B_{2} B_{1}}=(-1)^{B_{1}\left(A_{2}+B_{2}\right)} R_{B_{1}}^{A_{1}} S_{B_{2}}^{A_{2}} .
$$

Then two such matrices may be multiplied using normal matrix multiplication with no grading. For example

$$
(R \otimes S)^{A_{1} A_{2}}{ }_{B_{2} B_{1}}=\left(R^{\prime} \otimes S^{\prime}\right)^{A_{1} A_{2}} C_{2} C_{1}\left(R^{\prime \prime} \otimes S^{\prime \prime}\right)^{C_{1} C_{2}}{ }_{B_{2} B_{1}} .
$$

Essentially this convention moves the grading signs to the definition of the matrix tensor product. This convention is most convenient when dealing with the tensor product of two or more matrices. For example, the triple tensor product of two $2 \times 2$ matrices is an $8 \times 8$ matrix. Including the signs when writing these large matrices allows normal matrix multiplication, avoiding sign errors in the tedious manipulations. 


\section{Bibliography}

[1] V.G. Drinfeld, "Quantum Groups", Proc. Internat. Congr. Math., vol. 1, 798820, Berkeley (1986).

[2] M. Jimbo, Lett. Math. Phys. 11247 (1986).

[3] L.D. Faddeev, "Integrable Models in (1+1)-dimensional Quantum Field Theory", (Les Houches, 1982), Elsevier Science Publishers, Amsterdam, 1984.

[4] L. Alvarez-Gaumé, C. Gomez and G. Sierra, "Duality and Quantum Groups", CERN-TH.5369/89, UVGA-DPT-3/605/89, as well as earlier CERN and UVGA preprints.

[5] Yu.I. Manin, "Quantum Groups and Non-Commutative Geometry", Montréal University preprint, CRM-1561 (1988).

[6] L.D. Faddeev, N. Reshetikhin and L.A. Takhtajan, "Quantization of Lie Groups and Lie Algebras", LOMI E-14-87 (1987), to appear in M. Sato's 60 th birthday volume.

[7] L.A. Takhtajan, "Quantum Groups and Integrable Models", Advanced Studies in Pure Mathematics 19, 1989.

[8] S.L. Woronowicz, "Twisted SU(2) Group. An Example of Non-Commutative Differential Calculus", Publ. RIMS-Kyoto 23117 (1987).

[9] J. Wess and B. Zumino, "A Right-Invariant Calculus for $G L_{q}(2, C)^{\text {n }}$, to appear.

[10] M.J. Bowick and S.G. Rajeev, Phys. Rev. Lett. 58, 535 (1987); Nucl. Phys. B293, 348 (1987); The Complex Geometry of String Theory and Loop Space, 
Invited talks at the Eleventh Johns Hopkins Workshop on Current Problems in Particle Theory, Lanzhou, People's Republic of China, June 17-19, 1987.

[11] B. Zumino, The Geometry of the Virasoro Group for Physicists, Lectures presented at the Cargèse 1987 Summer School on Particle Physics, Cargèse, Corsica, August 3-21, 1987. LBL preprint LBL-24319, UCB preprint UCBPTH-87/48.

[12] K. Pilch and N.P. Warner, Journ. Classical and Quantum Gravity, MIT preprint, (CTP 1457, Feb. 1987).

[13] D. Harari, D.K. Hong, P. Ramond and V.G.J. Rodgers, Nucl. Phys. B294, 556 (1987).

[14] P. Oh and P. Ramond, Phys. Lett. B 195, 130 (1987).

[15] Z. Zhao, K. Wu and T. Saito, Phys. Lett. B 199, 37 (1987).

[16] W.B. Schmidke and S.P. Vokos, Ann. Phys. 189, 190 (1989).

[17] V. Jones, private communication.

[18] J. Schwenk, W.B. Schmidke and S. Vokos, "Properties of $2 \times 2$ Quantum Matrices in $\mathrm{Z}_{2}$-graded Spaces", preprint LBL-27933, UCB-PTH-89/25 (1989).

[19] S. Kobayashi and K. Nomizu, Foundations of Differential Geometry, (Interscience, New York, 1969), Vol. I, Chapter VI, Sec. 2,3. Vol. II, Chapter IV. This is a standard textbook on modern differential geometry.

[20] R. Coquereaux and A. Iadczyk, Riemannian Geometry, Fiber Bundles, Kaluza-Klein Theories and all that .... World Scientific (1988).

[21] S. Kobayashi, Differential Geometry of Complex Vector Bundles. Princeton University Press (1987).

[22] M.J. Bowick and A. Lahiri, J. Math. Phys. 29, 1979 (1988).

[23] B. DeWitt. Supermanifolds. Cambridge University Press (1984). 
[24] I.B. Frenkel, H. Garland and G.J. Zuckerman Proc. Natl. Acad. Sci. USA 83, 8442 (1986).

[25] D. Friedan, E. Martinec and S. Shenker, Nucl. Phys. B198, 93 (1986).

[26] M.B. Green, J.H. Schwarz and E. Witten. Superstring Theory. Cambridge University Press (1987). This textbook contains numerous references to original works. 

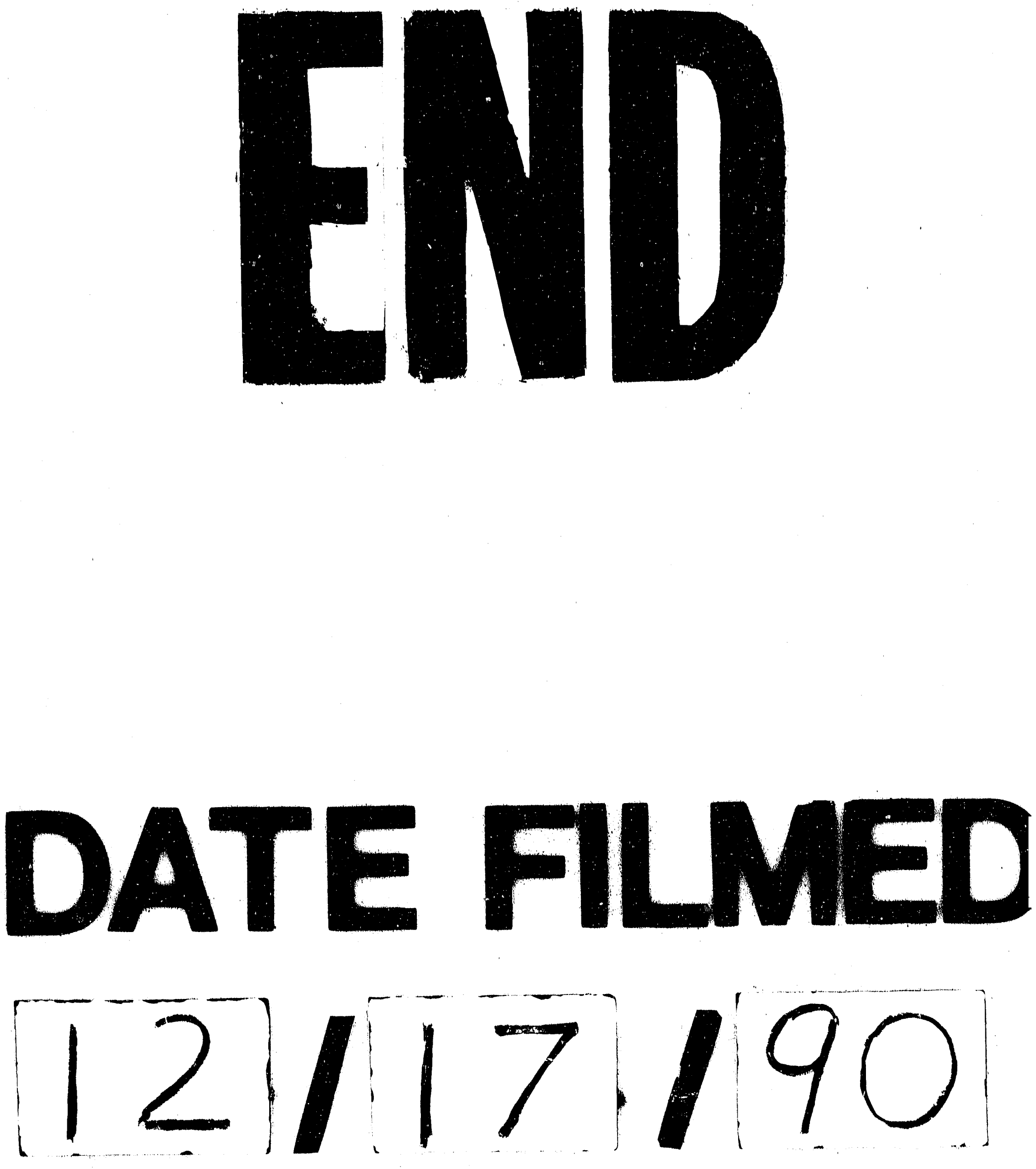
September 11, 1990

\title{
Differential Geometry of Groups in String Theory *
}

\author{
Ph.D. Thesis
}

\author{
William B. Schmidke, Jr. \\ Theoretical Physics Group \\ Physics Division \\ Lawrence Berkeley Laboratory \\ 1 Cyclotron Road \\ Berkeley, California 94720
}

\begin{abstract}
Techniques from differential geometry and group theory are applied to two topics from string theory. The first topic studied is quantum groups, with the example of GL(1|1). The quantum group $\mathrm{GL}_{q}(1 \mid 1)$ is introduced, and an exponential description is derived. The algebra and coproduct are determined using the invariant differential calculus method introduced by Woronowicz and generalized by Wess and Zumino. An invariant calculus is also introducel on the quantum superplane, and a representation of the algebra of $\mathrm{GL}_{q}(1 \mid 1)$ in terms of the super-plane coordinates is constructed.

The second topic follows the approach to string theory introduced by Bowick and Rajeev. Here the ghost contribution to the anomaly of the energy-momentum tensor is calculated as the Ricci curvature of the Kähler quotient space $\operatorname{Diff}\left(S^{1}\right) / S^{1}$. We discuss general Kähler quotient spaces and derive an expression for their Ricci curvatures. Application is made to the string and superstring diffeomorphism groups, considering all possible choices of subgroup. The formalism is extended to associated holomorphic vector bundles, where the Ricci curvature corresponds to the anomaly for different ghost sea levels.
\end{abstract}

- This work was supported by the Director, Office of Energy Research, Office of High Energy and Nuclear Physics, Division of High Energy Physics of the U.S. Department of Energy under Contract DE-AC03-76SF00038. 


\section{Acknowledgements}

I would like to thank my adviser, Professor Bruno Zumino, for giving me the opportunity to pursue this work and for his endless patierice with me. It has been a privelege to learn his elegant approach to physics.

I also thank Professor Marty Halpern whose excellent teaching inspired me to pursue this line of research. His support and encouragement have been a constant help.

I wish to thank the many other members of the theory group for advice and companionship throughout the course of this work. Special thanks to Betty Moura and Luanne Neumann for providing a warm and hospitable environment.

I also thank the many experimental physicists with whom I have collaborated over the course of my studies. Working with them has provided a well rounded physics education not common in the field these days.

My life in Berkeley has been enormously enriched by the wide variety of friends I have had over the years. Most of them fall into one of the following categories: the Sequoia residents; the Deadheads; the Texans; the Northside (Oakland) crowd; the Tuesday night softball group; and the Degenerate Vacua. I thank them all for their companionship which has kept me sane.

I dedicate this work to my parents, Helen and Bill Sr., whose love and support has made everything possible. 


\section{Contents}

1 Introduction 1

2 The Quantum Group $\mathrm{GL}_{\mathrm{q}}(1 \mid 1)$

2.1 R-matrix Quantization of Groups ................. . 4

2.2 R-matrix and q-relations for $\mathrm{GL}_{\mathrm{q}}(1 \mid 1) \ldots \ldots \ldots$

2.3 The Exponential Description . . . . . . . . . . . . . . 9

3 The Quantum Algebra GL $(1 \mid 1)$

3.1 Exterior Derivative and Cartan-Maurer Forms . . . . . . . . . 13

3.2 Additional q-relations . . . . . . . . . . . . . 15

3.3 The Algebra ......................... . . . 19

3.4 The Coproduct ....................... 21

3.5 Method of Faddeev, Reshetikhin, and Takhtajan . . . . . . . . . 24

4 The Quantum Super-Plane $\quad 28$

4.1 Coordinate and Differential q-relations ............ 28

4.2 Super-Plane Exterior Derivative ................ . 31

4.3 Additional q-relations . . . . . . . ......... 33

4.4 Derivative q-relations .................... 34 
4.5 Representation of Derivatives $\ldots \ldots \ldots \ldots \ldots$

4.6 Representation of the Algebra . . . . . . . . . . . 40

5 Quotient Space Geometry 43

5.1 Quotient Space $G / H \ldots \ldots \ldots \ldots \ldots \ldots \ldots$

5.2 Riemannian Geometry of $G / H \ldots \ldots \ldots \ldots \ldots$

5.3 Almost Complex Structure on $G / H \ldots \ldots \ldots \ldots$

5.4 Complex Structure on $G / H \ldots \ldots \ldots \ldots \ldots$

5.5 Kählerian Geometry of $G / H \ldots \ldots \ldots \ldots \ldots$

5.6 Discussion . . . . . . . . . . . . . . . 50

6 String Diffeomorphism Groups $\quad 52$

6.1 Quotient Spaces for the String . . . . . . . . . . . . . . . 52

6.2 Quoticnt Spaces for the Superstring . . . . . . . . . . . . 55

6.3 Gracled Algebras . . . . . . . . . . . . . . 56

7 Associated Vector Bundles $\quad 58$

7.1 Holomorphic Vector Bundles . . . . . . . . . . . . . . . 58

7.2 Implications for String Theory . . . . . . . . . . . . . . . 61

A Super-Tensor Index Conventions 63

A.1 Super-Functions and Derivatives . . . . . . . . . . . 63

A.2 Super-Vectors $\ldots \ldots \ldots \ldots \ldots \ldots \ldots \ldots \ldots$

A.3 Super-Tensors . . . . . . . . . . . . . . . . 67 


\section{Chapter 1}

\section{Introduction}

In recent years string theory has been dominated by the application of higher mathematics to theoretical physics. New mathematical structures unfamiliar to physicists have arisen in many studies. Mathematics well known to physicists has also been applied in new and powerful approaches. In this work we focus on the application of two branches of mathematics already common in theoretical physics: differential geometry and group theory. We will use techniques from both of these fields to study two different topics arising in string theory.

The first topic we discuss concerns quantum groups. Quantum groups arise naturally in several aspects of physics and mathematics, namely in the context of integrable models, quantum inverse scattering methods, the KnizhnikZamolodchikov equation, rational conformal field theory, as well as in the theory of Hopf algebras, solution of Yang-Baxter equations and theory of braids [1, 2, 3, 4]. For our purposes quantum groups can be thought of as one-parameter deformations of classical groups. We will concentrate on matrix groups. In this case the elements of a quantum matrix do not behave like ordinary c-numbers, but obey modified commutation rules imposed by the quantization procedure. Given these rules, the problem is to derive the algebra of the generators of the quantum group. For matrix groups one may also consider the vector space acted on by the matrices. The components of these vectors also obey modified commutation relations. This approach to quantum groups has been developed by Manin [5]. 
In this work we quantize the group GL(1|1). This is the group of nonsingular matrices acting on the superplane, a vector space with one commuting and one Grassmann coordinate. We quantize using the R-matrix procedure outlined in $[6$, 7]. Using the relations on matrix elements imposed by quantization, we determine the algebra of the generators. We follow the invariant differential calculus method introduced by Woronowicz [8] and generalized by Wess and Zumino [9]. This calculus is based on an exterior derivative which is nilpotent and obeys the Leibriz rule. The method also yields the coproduct, which describes how the generators act on a tensor product of spaces. Finally we construct an invariant differential calculus on the superplane. We also construct a representation of the quantum group generators based on the superplane coordinates.

The second main topic of this work concerns an approach to string theory initiated by Bowick and Rajeev [10]. They formulated open string theory in terms of holomorphic line bundles over the manifold $\operatorname{Diff}\left(\mathrm{S}^{1}\right) / \mathrm{S}^{1}$. This was motivated by the fact that the quantization procedure is not invariant under reparameterizations of the string coordinate. The open string may be described as a loop in phase space, and reparameterizations form the group of diffeomorphisms of the circle Diff $\left(\mathrm{S}^{1}\right)$. The action of $\operatorname{Diff}\left(\mathrm{S}^{1}\right)$ mixes creation and annihilation operators, changing the definition of the vacuum state. The subgroup of $\operatorname{Diff}\left(\mathrm{S}^{1}\right)$ consisting of the rigid rotations of the circle $\left.\cong U(1) \cong S^{1}\right)$ does not mix the operators. Thus, the inequivalent vacua may be identified with the quotient space $\mathcal{M} \equiv \operatorname{Diff}\left(\mathrm{S}^{1}\right) / \mathrm{S}^{1}$. $\mathcal{M}$ is an infinite dimensional Kähler manifold. Each fiber of the string vacuum bundle over $\mathcal{M}$ consists of the open string Fock space with the usual inner product. The curvature of this bundle is the anomaly in the Virasoro algebra. Geometrically, non-vanishing curvature means that a covariantly constant section of the bundle does not exist; one cannot define a reparameterization invariant vacuum. In reference [10] ghosts and their associated vacuum bundle are included, cancelling the anomaly in the critical dimension. The authors also noted that the curvature of the ghost vacuum bundle is equal to the curvature of the canonical line bundle over $\mathcal{M}$, which for a Kähler manifold is given by the Ricci form. This motivates our interest in the geometry of Diff( $\left(\mathrm{S}^{1}\right) / \mathrm{S}^{1}$ and related manifolds. Subsequent work has further clarified the Riemannian geometry of $\operatorname{Diff}\left(\mathrm{S}^{1}\right) / \mathrm{S}^{1}[11]$ and extended the 
calculation to the supersymmetric case super-Diff $\left(S^{1}\right) / S^{1}[12,13,14,15,16]$.

In this work we discuss the calculation of the curvature of the canonical line bundle of a quotient space and extend the formalism to include other levels of the ghost vacuum. The discussion uses the language of differential geometry familiar to physicists. We begin by reviewing the construction of a quotient space $\mathcal{M}=G / H$ for a group $G$ with subgroup $H$. The Riemannian geometry of such a space for $G$-invariant metrics is discussed. Next the conditions for $\mathcal{M}$ to be a complex manifold are outlined. If $M$ admits a Kähler metric the associated Ricci curvature has a simple form independent of the metric. These results are then applied to the groups relevant to the string and superstring, which are special cases of graded algebras. Here it is seen that the use of the Kähler metric regulates otherwise divergent quantities. Finally, the calculation of the Ricci curvature is extended from the tangent bundle of Riemann and Kähler geometry to more general vecto: bundles based on these spaces. This result corresponds to different filling levels of the ghost sea. 


\section{Chapter 2}

\section{The Quantum Group $\mathrm{GL}_{\mathrm{q}}(1 \mid 1)$}

In this chapter we discuss the quantum group $\mathrm{GL}_{\mathbf{q}}(1 \mid 1)$. The classical matrix group is quantized using the R-matrix procedure, following closely the discussion in [7]. This determines the bilinear quantum relations on the matrix elements. Covariance of these relations is demonstrated. The quantum determinant $: s$ so introduces. Finally, an exponential description of the quantum group is developed.

\section{$2.1 \quad R$-matrix Quantization of Groups}

The method of group quantization most commonly encountered for matrix groups, involves the $\mathrm{R}$-matrix. The $\mathrm{R}$-matrix is in the tensor product of two matrix spaces. It describes how to reverse the ordering of the same matrix in two different tensor spaces. Explicitly, if the same matrix $A$ is written as $A_{1}=A \otimes 1$ when in the first space and $A_{2}=1 \otimes A$ in the second, then the R-matrix equation is

$$
R A_{1} A_{2}=A_{2} A_{1} R
$$

This equation defines bilinear relations on the elements of $A$. We will refer to such relations defined by quantization as $q$-relations.

If one considers the triple tensor product of matrices, consistency determines a constraint in the R-matrix. Let $R_{(12)}$ act on the 1 and 2 matrix spaces, $R_{(13)}$ on the 1 and 3 spaces, and $R_{(23)}$ on the 2 and 3 spaces. The $R$-matrix commutes 
with matrices not in the space it acts on. Then using the above $R$-matrix equation repeatedly to switch matrices shows

$$
\begin{aligned}
R_{(23)} R_{(13)} R_{(12)} A_{1} A_{2} A_{3} & =R_{(23)} R_{(13)} A_{2} A_{1} A_{3} R_{(12)} \\
& =R_{(23)} A_{2} A_{3} A_{1} R_{(13)} R_{(12)} \\
& =A_{3} A_{2} A_{1} R_{(23)} R_{(13)} R_{(12)}
\end{aligned}
$$

and we see that $R_{(23)} R_{(13)} R_{(12)}$ reverse the order of $A_{1} A_{2} A_{3}$ to $A_{3} A_{2} A_{1}$. However, the order in which we swapped matrices can be changed. The other way is

$$
\begin{aligned}
R_{(12)} R_{(13)} R_{(23)} A_{1} A_{2} A_{3} & =R_{(12)} R_{(13)} A_{1} A_{3} A_{2} R_{(23)} \\
& =R_{(12)} A_{3} A_{1} A_{2} R_{(13)} R_{(23)} \\
& =A_{3} A_{2} A_{1} R_{(12)} R_{(13)} R_{(23)}
\end{aligned}
$$

Here we see that $R_{(12)} R_{(13)} R_{(23)}$ switches the ordering as before. Thus these two triple products of $R$-matrices are equal:

$$
R_{(12)} R_{(13)} R_{(23)}=R_{(23)} R_{(13)} R_{(12)}
$$

This is the Yang-Baxter equation. No further constraints arise from considering higher tensor products.

\subsection{R-matrix and q-relations for $\mathrm{GL}_{\mathrm{q}}(1 \mid 1)$}

The classical group GL(1/1) is the group of $2 \times 2$ non-sigular super-matrices which act on a 2-dimensional vector space with one commuting and one anticommuting coordinate. Such a matrix $A \in \mathrm{GL}(1 \mid 1)$ can be written

$$
A=\left(\begin{array}{ll}
a & \beta \\
\gamma & d
\end{array}\right)
$$

where the matrix elements $a$ and $d$ are commuting and $\beta$ and $\gamma$ are anticommuting. The (anti-)commutation rules will be modified by quantization.

The $\mathrm{R}$-matrix for $\mathrm{GL}_{\mathrm{q}}(1 \mid 1)$ is in the tensor product of $2 \times 2$ super-matrices, so it is a $4 \times 4$ matrix. As discussed in the appendix, it is convenient to choose 
an index ordering convention which allows simple matrix multiplcation with no additional grading signs. We choose the indices ordered as $R^{i_{1} i_{2}}{ }_{j_{2} j_{1}}$, where the subscripts on the indices indicate which vector space in the tensor product the index belongs to. The the $\mathrm{R}$-matrix for $\mathrm{GL}_{\mathrm{q}}(1 \mid 1)$ is

$$
R=\left(\begin{array}{cccc}
q & 0 & 0 & 0 \\
0 & 1 & 0 & 0 \\
0 & \lambda & 1 & 0 \\
0 & 0 & 0 & q^{-1}
\end{array}\right)
$$

where $\lambda=q-q^{-1}$. This $\mathrm{R}$-matrix has been used extensively in the work of Lee and Couture [17].

It can be verified that this $R$-matrix satisfies the Yang-Baxter equation (2.2). For this the R-matrix is written as an $8^{*} \times 8$ matrix in the tensor product of $3.2 \times 2$ super-matrices. Again it is convenient to choose index ordering for simple matrix multiplication. In terms of the above index ordering for $R$, we have

$$
\begin{aligned}
& R_{(12)}^{i_{1} i_{2} i_{3}}{ }_{j 3 j_{2} j_{1}}=R^{i_{1} i_{2}}{ }_{j_{2} j_{1}} \delta^{i_{3}}{ }_{j_{3}} \\
& R_{(13){ }^{1} j_{3} j_{2} j_{1}}^{i_{1} i_{3}}=(-1)^{i_{2}\left(i_{3}+j_{3}\right)} R^{i_{1} i_{3}}{ }_{j_{3} j_{1}} \delta^{i_{2}}{ }_{j_{2}} \\
& R_{(23){ }^{i_{2} i_{3} i_{3}}{ }_{j} j_{2} j_{1}}=(-1)^{i_{1}\left(i_{2}+i_{3}+j_{2}+j_{3}\right)} R^{i_{2} i_{3}}{ }_{j_{3} j_{2}} \delta^{i_{1}}{ }_{j_{1}} .
\end{aligned}
$$

Here the $Z_{2}$ grading signs are $(-1)^{i}=+1$ for index $i$ corresponding to the first row or column of a $2 \times 2$ matrix space, and $(-1)^{i}=-1$ for $i$ correspondine to the second row or column.

Having verified that the R-matrix satisfies the Yang-Baxter equation. we can now use it in (2.1) to determine q-relations on the elements of a matrix $A$ as in (2.3). In writing the $4 \times 4$ matrices corresponding to $A_{1} A_{2}$ and $A_{2} A_{1}$ we once again choose index ordering for simple matrix multiplication. Then the grading sigus are given by

$$
\begin{aligned}
& \left(A_{1}\right)^{i_{1} i_{2}} j_{2} j_{1}=(A \otimes 1)^{i_{1} i_{2}}{ }_{j 2} j_{1}=(-1)^{j_{1}\left(i_{2}+j_{2}\right)} A^{i_{1}}{ }_{j_{1}} \delta^{i_{2}}{ }_{j_{2}} \\
& \left(A_{2}\right)^{i_{1} i_{2}}{ }_{j_{2} j_{1}}=(1 \otimes A)^{i_{1} i_{2}}{ }_{j 2 j_{1}}=(-1)^{i_{1}\left(i_{2}+j_{2}\right)} A^{i_{2}}{ }_{j_{2}} \delta^{i_{1}}{ }_{j_{1}}
\end{aligned}
$$


so the $4 \times 4$ matrices are

$$
A_{1}=\left(\begin{array}{llll}
a & 0 & \beta & 0 \\
0 & a & 0 & \beta \\
\gamma & 0 & d & 0 \\
0 & \gamma & 0 & d
\end{array}\right) \quad A_{2}=\left(\begin{array}{cccc}
a & \beta & 0 & 0 \\
\gamma & d & 0 & 0 \\
0 & 0 & a & -\beta \\
0 & 0 & -\gamma & d
\end{array}\right) .
$$

Using these matrices and the R-matrix (2.4) in equation (2.1) gives bilinear $:-$ relations for the elements of $A$. The independent q-relations are

$$
\begin{array}{ll}
a \beta=q \beta a & \beta^{2}=0 \\
a \gamma=q \gamma a & \gamma^{2}=0 \\
d \beta=q \beta d & \beta \gamma=-\gamma \beta \\
d \gamma=q \gamma d & a d-d a=-\lambda \beta \gamma
\end{array}
$$

where $\lambda=q-q^{-1}$. Note that the first three relations in the right column are the same as in the classical $(q=1)$ case. The other relations are modified by quantization.

The determinant of the quantum matrix $A$ may be defined in the same way as for a classical matrix. Begin by decomposing the matrix into a product of a lower triangular matrix, a diagonal matrix, and an upper triangular matrix as follows:

$$
A=\left(\begin{array}{ll}
a & \beta \\
\gamma & d
\end{array}\right)=\left(\begin{array}{cc}
1 & 0 \\
\gamma a^{-1} & 1
\end{array}\right)\left(\begin{array}{cc}
a & 0 \\
0 & d-\gamma a^{-1} \beta
\end{array}\right)\left(\begin{array}{cc}
1 & a^{-1} \beta \\
0 & 1
\end{array}\right) .
$$

The determinant of $A$ is the product of the deter.ninants of each of these three matrices. The determinants of the triangular matrices are one. The determinant of a diagonal super-matrix is the product of the diagonal elements in bosonic rows divided by the diagonal elements in fermionic rows. This is a result of the fact that the super-trace of a super-matrix is the sum of the bosonic diagonal elements minus the fermionic elements. The determinant of $A$ is then the exponential of the super-trace of $\ln A$. (Later we will demonstrate that this is also true for these quantum matrices.)

Using the above decomposition and rule for determinant of diagonal supermatrix gives

$$
\operatorname{det}(A)=a\left(d-\gamma a^{-1} \beta\right)^{-1}
$$


The term in parerivineses is easy to invert since the Grassmann elements are nilpotent. Then using the q-relations to rearrange the group parameters (note that no net factors of $q$ appear) we find

$$
\begin{aligned}
\operatorname{det}(A) & =a d^{-1}-\beta d^{-1} \gamma d^{-1} \\
& =d^{-1} a-d^{-1} \beta d^{-1} \gamma .
\end{aligned}
$$

This can be shown to obey the usual properties of the determinant, for example $\operatorname{det}(A) \operatorname{det}(B)=\operatorname{det}(A B)$. More importantly, the determinant is central, i.e. it commutes with all of the matrix elements:

$$
\operatorname{det}(A) A=A \operatorname{det}(A) .
$$

Later we will also consider the group $\mathrm{SL}_{\mathrm{q}}(1 \mid 1)$, the group of $2 \times 2$ super-matrices with determinant one.

One interesting property of the quantum groups is covariance of the qrelations (2.5). This means that the product of two different quantum matrices with elements satisfying the q-relations (2.5) produces a new quantum matrix with elements satisfying the same relations. Explicitly, consider two matrices $A$ and $A^{\prime}$ with elements

$$
A=\left(\begin{array}{ll}
a & \beta \\
\gamma & d
\end{array}\right) \quad A^{\prime}=\left(\begin{array}{ll}
a^{\prime} & \beta^{\prime} \\
\gamma^{\prime} & d^{\prime}
\end{array}\right) .
$$

The elements of each matrix satisfy the q-relations (2.5), e.g. $a \beta=q \beta a, a^{\prime} \beta^{\prime}=$ $q \beta^{\prime} a^{\prime}$, etc. The elemenmts of $A$ (anti-)commuic with those of $A^{\prime}$. The product matrix $A^{\prime \prime}$ is defined by

$$
A^{\prime \prime}=A A^{\prime}=\left(\begin{array}{ll}
a^{\prime \prime} & \beta^{\prime \prime} \\
\gamma^{\prime \prime} & d^{\prime \prime}
\end{array}\right) .
$$

It is straightforward to check that the elements of $A^{\prime \prime}$ given by

$$
\begin{array}{ll}
a^{\prime \prime}=a a^{\prime}+\beta \gamma^{\prime} . & \beta^{\prime \prime}=a \beta^{\prime}+\beta d^{\prime} \\
\gamma^{\prime \prime}=\gamma a^{\prime}+d \gamma^{\prime} & d^{\prime \prime}=\gamma \beta^{\prime}+d d^{\prime}
\end{array}
$$

also satisfy the q-relations, e.g. $a^{\prime \prime} \beta^{\prime \prime}=q \beta^{\prime \prime} a^{\prime \prime}$. It is in this sense that one says that the quantum group $G_{L_{q}}(1 \mid 1)$ is closed under multiplication. The product of two different elements of $\mathrm{GL}_{\mathbf{q}}(1 \mid 1)$ produces another element of $\mathrm{GL}_{\mathbf{q}}(1 \mid 1)$. 


\subsection{The Exponential Description}

Another interesting property of the quantum group $\mathrm{GL}_{\mathrm{q}}(1 \mid 1)$ is found when considering powers of an element of the group. Consider the matrix $A^{2}$ :

$$
A^{2}=A A=\left(\begin{array}{ll}
a_{2} & \beta_{2} \\
\gamma_{2} & d_{3}
\end{array}\right)
$$

with elements

$$
\begin{array}{ll}
a_{2}=a^{2}+\beta \gamma & \beta_{2}=\alpha \beta+\beta d \\
\gamma_{2}=\gamma a+d \gamma & d_{2}=\gamma \beta+d^{2} .
\end{array}
$$

It is straightforward to verify that the elements of $A^{2}$ satisfy the q-relations (2.5) with $q$ replaced by $q^{2}$, or explicitly

$$
\begin{array}{ll}
a_{2} \beta_{2}=q^{2} \beta_{2} a_{2} & \beta_{2}^{2}=0 \\
a_{2} \gamma_{2}=q^{2} \gamma_{2} a_{2} & \gamma_{2}^{2}=0 \\
d_{2} \beta_{2}=q^{2} \beta_{2} d_{2} & \beta_{2} \gamma_{2}=-\gamma_{2} \beta_{2} \\
d_{2} \gamma_{2}=q^{2} \gamma_{2} d_{2} & a_{2} d_{2}-d_{2} a_{2}=-\left(q^{2}-q^{-2}\right) \beta_{2} \gamma_{2} .
\end{array}
$$

This means that if $A \in \mathrm{GL}_{\mathrm{q}}(1 \mid 1)$, then $A^{2} \in \mathrm{GL}_{\mathrm{q}^{2}}(1 \mid 1)$. This suggests that similar relations will hold for any power of $A$. This has been verified through detailed calculations as discussed in [18]. Here we will demonstrate this through a differen: approach.

Because the group $\mathrm{GL}_{\mathrm{q}}(1 \mid 1)$ is relatively simple, it is possible to explicitly check many of its properties. Here we will consider an exponential description of group elements. Write an element $A$ of $\mathrm{GL}_{\mathbf{q}}(1 \mid 1)$ as the exponential of another matrix $M$ times a parameter $h$ defined by

$$
A=e^{h M} \quad q=e^{h} .
$$

The elements of $A$ and $M$ are

$$
A=\left(\begin{array}{ll}
a & \beta \\
\gamma & d
\end{array}\right) \quad M=\left(\begin{array}{ll}
r & \sigma \\
\tau & u
\end{array}\right) .
$$

Like the elements of $A$, the elements of $M$ will not obey ordinary graded commutation rules. The correct relations can be determined by writing the elements of 
$A$ to order $h^{2}$ and plugging into the q-relations (2.5). One finds that the elements of $M$ must obey

$$
\begin{array}{ll}
r \sigma-\sigma r=\sigma & r u=u r \\
u \sigma-\sigma u=\sigma & \sigma \tau=-\tau \sigma \\
r \tau-\tau r=\tau & \sigma^{2}=0 \\
u \tau-\tau u=\tau & \tau^{2}=0 .
\end{array}
$$

The relations in the right column are the usual graded commutation relations; the left column is modified. Note that $q$ (or $h$ ) does not appear in these relations. Also note that the super-trace of $M,(r-u)$, commutes with all of the elements.

Using these relations, an explicit form of the exponential can be written. This is easy to do because of the nilpotency of $\sigma$ and $\tau$ in (2.8). Write $M$ as a sum of two matrices as follows:

$$
M=M_{0}+M_{1} \quad M_{0}=\left(\begin{array}{cc}
r & 0 \\
0 & u
\end{array}\right) \quad M_{1}=\left(\begin{array}{ll}
0 & \sigma \\
\tau & 0
\end{array}\right) .
$$

Because of nilpotency, $M_{1}^{n>2}$ yanishes. Then any power of $M$ greater than or equal to two may be written

$$
M^{n \geq 2}=M_{0}^{n}+\sum_{m=0}^{n-1} M_{0}^{m} M_{1} M_{0}^{n-m-1}+\sum_{m=0}^{n-2} \sum_{l=0}^{n-m-2} M_{0}^{m} M_{1} M_{0}^{l} M_{1} M_{0}^{n-m-l-2} .
$$

Using the relations (2.8), the terms in the summations can be written in a simple form. The needed expressions are

$$
\begin{aligned}
& M_{0}^{n}=\left(\begin{array}{cc}
r^{n} & 0 \\
0 & u^{n}
\end{array}\right) \\
& M_{0}^{m} M_{1} M_{0}^{l}=\left(\begin{array}{cc}
0 & \sigma(r+1)^{m} u^{l} \\
\tau(u+1)^{m} r^{l} & 0
\end{array}\right) \\
& M_{0}^{m} M_{1} M_{0}^{l} M_{1} M_{0}^{k}=\left(\begin{array}{cc}
\sigma \tau(r+2)^{m}(u+1)^{l} r^{k} & 0 \\
0 & -\sigma \tau(u+2)^{m}(r+1)^{l} u^{k}
\end{array}\right) .
\end{aligned}
$$

Using these expressions in the expansion of $M^{n}$, the summations can be performed leaving

$$
M^{n}=\left(\begin{array}{ll}
r_{n} & \sigma_{n} \\
\tau_{n} & u_{n}
\end{array}\right)
$$


where

$$
\begin{aligned}
& r_{n}=r^{n}+\sigma \tau\left(\frac{(r+2)^{n}}{2(1+r-u)}+\frac{r^{n}}{2(1-r+u)}-\frac{(u+1)^{n}}{1-(r-u)^{2}}\right) \\
& \sigma_{n}=\sigma \frac{(r+1)^{n}-u^{n}}{1+r-u} \\
& \tau_{n}=\tau \frac{(u+1)^{n}-r^{n}}{1-r+u} \\
& u_{n}=u^{n}-\sigma \tau\left(\frac{(u+2)^{n}}{2(1-r+u)}+\frac{u^{n}}{2(1+r-u)}-\frac{(r+1)^{n}}{1-(r-u)^{2}}\right) .
\end{aligned}
$$

Finally, the exponential sum

$$
e^{h M}=\sum_{n=0}^{\infty} \frac{h^{n}}{n !} M^{n}
$$

may be performed, yielding the elements of the matrix $A$. One finds

$$
\begin{aligned}
& a=e^{h r}+\frac{\sigma \tau}{1-(r-u)^{2}}\left(\frac{1}{2}(1-r+u) e^{h(r+2)}+\frac{1}{2}(1+r-u) e^{h r}-e^{h(u+1)}\right) \\
& \beta=\frac{\sigma}{1+r-u}\left(e^{h(r+1)}-e^{h u}\right) \\
& \gamma=\frac{\tau}{1-r+u}\left(e^{h(u+1)}-e^{h r}\right) \\
& d=e^{h u}-\frac{\sigma \tau}{1-(r-u)^{2}}\left(\frac{1}{2}(1+r-u) e^{h(u+2)}+\frac{1}{2}(1-r+u) e^{h u}-e^{h(r+1)}\right) .
\end{aligned}
$$

Note that these expressions have been written with no explicit appearance of $q$. All dependence on $g$ is carried by $h$.

It is straightforward to verify, using (2.8), that the above expressions for the elements of $A$ satisfy the q-relations (2.5), with $q=e^{h}$. Now consider $A^{p}$, where $p$ is any $c$-number. From (2.7) we see that this may be written

$$
A^{p}=\left(e^{h M}\right)^{p}=e^{p h M} .
$$

Then the elements of $A^{p}$ :

$$
A^{p}=\left(\begin{array}{ll}
a_{p} & \beta_{p} \\
\gamma_{p} & d_{p}
\end{array}\right)
$$

are obtained from (2.9) with the substitution $h \rightarrow p h$, with the elements of $M$ $\left(r, \sigma, \tau\right.$, and $u$ ) still satisfying (2.8). The q-relations for the elements of $A^{p}$ are 
satisfied with the same substitution, with $q \rightarrow q^{p}$. Explicitly,

$$
\begin{array}{lll}
a_{p} \beta_{p}=q^{p} \beta_{p} a_{p} & d_{p} \beta_{p}=q^{p} \beta_{p} d_{p} & \beta_{p} \gamma_{p}=-\gamma_{p} \beta_{p} \\
a_{p} \gamma_{p}=q^{p} \gamma_{p} a_{p} & d_{p} \gamma_{p}=q^{p} \gamma_{p} d_{p} & a_{p} d_{p}-d_{p} a_{p}=-\left(q^{p}-q^{-p}\right) \beta_{p} \gamma_{p}
\end{array}
$$

and $\beta_{p}$ and $\gamma_{p}$ are nilpotent as usual. This verifies the property of $\mathrm{GL}_{q}(1 \mid 1)$ suggested by $A^{2}$ mentioned earlier. If $A \in \mathrm{GL}_{\mathrm{q}}(1 \mid 1)$, then $A^{p} \in \mathrm{GL}_{\mathrm{qp}}(1 \mid 1)$.

Another interesting property of $\mathrm{GL}_{\mathrm{q}}(1 \mid 1)$ is seen by writing the deteiminani (2.6) using the exponential representation. Since the variables $\sigma$ and $\tau$ are nilpotent it is straightforward to show that the inverse of $d$ is given by

$$
d^{-1}=e^{-h u}+\frac{\sigma \tau}{1-(r-u)^{2}}\left(\frac{1}{2}(1-r+u) e^{-h(u+2)}+\frac{1}{2}(1+r-u) e^{-h u}-e^{h(r-2 u-1)}\right) .
$$

Using this expression and (2.9) in the definition of the determinant (2.6), one finds

$$
\operatorname{det}(A)=\left(a-\beta d^{-1} \gamma\right) d^{-1}=e^{h(r-u)} .
$$

The exponent $(r-u)$ is the super-trace of $M$, which is central. Thus we have the general relation

$$
\ln \operatorname{det}(A)=s-\operatorname{tr} \ln (A) .
$$

This coincides with the relation for classical matrices. 


\section{Chapter 3}

\section{The Quantum Algebra $\mathrm{GL}_{\mathrm{q}}(1 \mid 1)$}

In this chapter we determine the algebra of $\mathrm{GL}_{\mathbf{q}}(1 \mid 1)$. We use the method of Wess and Zumino [9] based on an invariant differential calculus. This is a generalizatiok of the work ' Woronowicz [8]. Fundamental to this method is an exterior derivative which is nilpotent and obeys the Leibniz rule. Input to the method are the q-relations defined by the R-matrix in (2.1). The elements of a matrix in the group are the group parameters. The method derives the algebra of cenerators of infinitessimal deformations of the group parameters. It also determines the coproduct of the generators.

Here we apply this methor' to $G L_{q}(1 \mid 1)$, starting with the q-relations (2.5). We find the algebra of $\mathrm{GL}_{\mathrm{q}}(1 \mid 1)$ and its subalgebro $\mathrm{SL}_{\mathbf{q}}(1 \mid 1)$, and the coproduct of the generators. Our results are also shown to be consistent with the method of Faddeev, Reshetikhin, and Takhtajan [6, 7].

\subsection{Exterior Derivative and Cartan-Maurer Forms}

We begin by defining the exterior derivative $\delta$. We require $\delta$ to be nilpotent and to obey the (graded) Leibniz rule:

$$
\begin{aligned}
& \delta^{2}=0 \\
& \delta(F G)=(\delta F) G+(-1)^{F} F(\delta G) .
\end{aligned}
$$


Here $(-1)^{F}=+1(-1)$ if $F$ is commuting (anticommuting). This grading is required since $\delta$ is anticommuting.

Next define right-invariant Cartan-Maurer 1-forms in the usual way. For a group element $A$ define:

$$
\Omega=(\delta A) A^{-1}=\left(\begin{array}{cc}
\omega_{1} & \nabla_{+} \\
\nabla_{-} & \omega_{2}
\end{array}\right) .
$$

Here the 1-forms $\omega_{1}$ and $\omega_{2}$ are anticommuting while $v_{+}$and $v_{-}$are commutin $\tilde{E}$. Under right multiplication by a constant group element $A_{0}$ obeying $\delta A_{0}=0$,

$$
\begin{aligned}
& A^{\prime}=A A_{0} \\
& \Omega^{\prime}=\left(\delta A^{\prime}\right)\left(A^{\prime}\right)^{-1}=(\delta A) A^{-1}=\Omega
\end{aligned}
$$

and we see that the Cartan-Maurer forms are right-invariant.

By manipulating the definition of $\Omega$ one can determine the action of the exterior derivative on the group parameters and on the Cartan-Maurer forms. Rearranging (3.2) gives $\delta A=\Omega A$ or for each group parameter

$$
\begin{array}{ll}
\delta a=\omega_{1} a+v_{+} \gamma & \delta \beta=\omega_{1} \beta+v_{+} d \\
\delta \gamma=\omega_{2} \gamma+v_{-} a & \delta d=\omega_{2} d+v_{-} \beta .
\end{array}
$$

Acting on (3.2) with $\delta$ and using (3.1) we have

$$
\delta \Omega=\tilde{\Omega} \Omega \quad \tilde{\Omega}=\left(\begin{array}{cc}
\omega_{1} & -\nabla_{+} \\
-\nabla_{-} & \omega_{2}
\end{array}\right) .
$$

The signs in $\tilde{\Omega}$ are determined by the grading in the Leibniz rule (3.1). Then we find for each 1 -form

$$
\begin{array}{ll}
\delta \omega_{1}=\omega_{1}^{2}-v_{+} v_{-} & \delta v_{+}=\omega_{1} v_{+}-v_{+} \omega_{2} \\
\delta v_{-}=\omega_{2} v_{-}-v_{-} \omega_{1} & \delta \omega_{2}=\omega_{2}^{2}-v_{-} v_{+} .
\end{array}
$$

These are the usual (graded) Cartan-Maurer equations. Note that they are written with the ordering defined by (3.4). This is because we do not yet know how to cummute 1-forms; they may obey q-relations which differ from classical (antijcommutators. 
The exterior derivative may be expanded as a sum of the 1-forms multiplying the generators of the algebra:

$$
\delta=\omega_{1} T_{1}+\omega_{2} T_{2}+v_{+} \nabla_{+}+v_{-} \nabla_{-}
$$

The generators $T_{1}$ and $T_{2}$ are commuting and $\nabla_{+}$and $\nabla_{-}$are anticommuting so that overall $\delta$ is anticommuting. From (3.3) one can read off the action of the generators on the group parameters (e.g. $T_{1} a=a$ and $\nabla_{+} z=\gamma$, etc.) The algebra of the generators may be determined using the nilpotecy and Leibniz ivle "(3.1) obeyed by the exterior derivative. Applying $\delta$ to the above expausion (3.6) gives (sum $i=1,2$ )

$$
\begin{aligned}
\delta= & \omega_{i} T_{i}+v_{ \pm} \nabla_{ \pm} \\
\delta^{2}= & \left(\delta \omega_{i}\right) T_{i}+\left(\delta v_{ \pm}\right) \nabla_{ \pm}-\omega_{i} \delta T_{i}+v_{ \pm} \delta \nabla_{ \pm} \\
= & \left(\omega_{1}^{2}-v_{+} v_{-}\right) T_{1}-\omega_{1}\left(\omega_{i} T_{i}+v_{ \pm} \nabla_{ \pm}\right) \\
& +\left(\omega_{2}^{2}-v_{-} v_{+}\right) T_{2}-\omega_{2}\left(\omega_{i} T_{i}+v_{ \pm} \nabla_{ \pm}\right) \\
& +\left(\omega_{1} v_{+}-v_{+} \omega_{2}\right) v_{+}+v_{+}\left(\omega_{i} T_{i}+v_{ \pm} \nabla_{ \pm}\right) \\
& +\left(\omega_{2} v_{-}-v_{-} \omega_{1}\right) v_{-}+v_{-}\left(\omega_{i} T_{i}+v_{ \pm} \nabla_{ \pm}\right) \\
& =0 .
\end{aligned}
$$

If we knew how to commute 1-forms, we could rearrange this expansion, with the coefficients of the 1-forms yielding the algebra of generators. Therefore we must determine q-relations between the Cartan-Maurer forms. With this information we can return to this expansion and derive the alge bra.

\subsection{Adcitional q-relations}

First we wish to extend the q-relations between group parameters (2.5) to include q-relations between Cartan-Maurer forms and group parameters. Recall that the Cartan-Maurer forms are right invariant. We shall require the new q-relations to also be right invariant. (This is already true for the relations (2.5)). Under right 
multiplication $A^{\prime}=A A_{0}$ the group parameters transform to

$$
\begin{array}{ll}
a^{\prime}=a a_{0}+\beta \gamma_{0} & \beta^{\prime}=x \beta_{0}+\beta d_{0} \\
\gamma^{\prime}=\gamma a_{0}+d \gamma_{0} & d^{\prime}=\gamma \beta_{0}+d d_{0} .
\end{array}
$$

Under this transformation, $a$ and $\beta$ mix together, and $d$ and $\gamma$ mix. Thus, in order to be right invariant, $a$ and $\beta$ should have the same q-relations with the 1 -forms, and $d$ and $\gamma$ should have the same q-relations (up to $Z_{2}$ grading signs.) We sh:" consider the following ansatz:

$$
\begin{array}{ll}
a\left(s^{i} \omega_{i}+\sigma^{ \pm} v_{ \pm}\right)=\left(t^{i} \omega_{i}+\tau^{ \pm} v_{ \pm}\right) a & d\left(s^{\prime i} \omega_{i}+\sigma^{ \pm \pm} v_{ \pm}\right)=\left(t^{\prime i} \omega_{i}+\tau^{ \pm} v_{ \pm}\right) d \\
\beta\left(s^{i} \omega_{i}+\sigma^{ \pm} v_{ \pm}\right)=-\left(t^{i} \omega_{i}+\tau^{ \pm} v_{ \pm}\right) \beta & \gamma\left(s^{i} \omega_{i}+\sigma^{\prime \pm} v_{ \pm}\right)=-\left(t^{\prime i} \omega_{i}+\tau^{\prime \pm} v_{ \pm}\right) \gamma
\end{array}
$$

where $s^{i}$ etc. define the form mixing. Next, recall that the determinant $D=\operatorname{det} A$ defined in (2.6) is central, i.e. it commutes with all of the group parameters. We wish to extend this to the algebra with Cartan-Maurer forms:

$$
\begin{gathered}
D \omega_{1,2}=\omega_{1,2} D \\
D v_{ \pm}=v_{ \pm} D .
\end{gathered}
$$

Since $D=a d^{-1}-\beta d^{-1} \gamma d^{-1}, a$ and $d^{-1}$ must have inverse mixing in (3.8), or $a$ and $d$ must have the same mixing, with primed coefficients equal to unprimed ones. Then all group parameters have the same q-relations with the 1-forms (up to grading signs). For a general group parameter $A_{r} \in\{a, \beta, \gamma, d\}$ we write

$$
A_{r}\left(s^{i} \omega_{i}+\sigma^{ \pm} v_{ \pm}\right)=(-1)^{A_{r}}\left(t^{i} \omega_{i}+\tau^{ \pm}\right) A_{r} .
$$

Here $(-1)^{A_{r}}=+1$ for $A_{r}=a$ or $d$ and $(-1)^{A_{r}}=-1$ for $A_{r}=\beta$ or $\gamma$.

We now attempt to determine these relations by applying $\delta$ to the qrelations (2.5). Beginning with the simplest relation $\beta^{2}=0$ we find

$$
\begin{aligned}
\delta \beta^{2} & =(\delta \beta) \beta-\beta(\delta \beta) \\
& =\omega_{1} \beta \beta+v_{+} d \beta-\beta \omega_{1} \beta-\beta v_{+} d \\
& =\left(q v_{+} \beta-\beta v_{+}\right) d \\
& =0 .
\end{aligned}
$$


The first and third terms in the second line vanish since they are proportional to $\beta^{2}$. The third line is obtained from the second using $d \beta=q \beta d$. Since this must vanish for all values of the group parameters, the coefficient of $d$ in the third line must vanish, yielding $\beta v_{+}=q v_{+} \beta$. Following the same procedure on $\gamma^{2}=0$ gives

$$
\delta \gamma^{2}=\left(q v_{-} \gamma-\gamma v_{-}\right) a=0 .
$$

Recalling that all group parameters have the same q-relations with the CartanMaurer forms, we summarize

$$
A_{r} v_{ \pm}=q v_{ \pm} A_{r}
$$

We see that the 1 -forms $v_{ \pm}$obey simple q-relations with the group parameters, with no mixing between 1-forms.

Acting on the relation $\beta \gamma=-\gamma \beta$ with $\delta$ gives

$$
\delta(\beta \gamma+\gamma \beta)=\left(\omega_{1}-\omega_{2}\right) \beta \gamma+\beta\left(\omega_{1}-\omega_{2}\right) \gamma=0
$$

which is solved by $\left(\omega_{1}-\omega_{2}\right) \beta=-\beta\left(\omega_{1}-\omega_{2}\right)$ or in general

$$
A_{r}\left(\omega_{1}-\omega_{2}\right)=(-1)^{A_{r}}\left(\omega_{1}-\omega_{2}\right) A_{r} .
$$

Here we see that the linear combination $\left(\omega_{1}-\omega_{2}\right)$ is an eigenvector under commutation with eigenvalue 1 . Suppose there is another linear combination with eigenvalue $f$. Define the 1-forms

$$
\begin{aligned}
& \omega_{y}=\frac{1}{2}\left(\omega_{1}-\omega_{2}\right) \\
& \omega_{X}=\frac{1}{2}\left((1+\phi) \omega_{1}+(1-\phi) \omega_{2}\right)
\end{aligned}
$$

which obey the q-relations

$$
\begin{aligned}
& A_{r} \omega_{Y}=(-1)^{A_{r}} \omega_{Y} A_{r} \\
& A_{r} \omega_{X}=(-1)^{A_{r}} f \omega_{X} A_{r} .
\end{aligned}
$$

Further application of $\delta$ to the remaining q-relations in (2.5) yields no new information. We are left with two undetermined parameters, $f$ and $\phi$, both related to $\omega_{X}$. 
Next we wish to determine q-relations between the Cartan-Maurer forms themselves. Again we apply $\delta$, this time to the relations $(3.10,3.12)$. Starting with the q-relations between $v_{+}$and $a$ and $d$ gives

$$
\begin{gathered}
\delta\left(a v_{+}-q v_{+} a\right)=q\left(\left(f \omega_{X}+(1-\phi) \omega_{Y}\right) v_{+}-v_{+}\left(f \omega_{X}+(1-\phi) \omega_{Y}\right)\right) a=0 \\
\delta\left(d v_{+}-q v_{+} d\right)=q\left(\left(f \omega_{X}-(1+\phi) \omega_{Y}\right) v_{+}-v_{+}\left(f \omega_{X}-(1+\phi) \omega_{Y}\right)\right) d \\
+q\left(v_{-} v_{+}-v_{+} v_{-}\right) \beta=0 .
\end{gathered}
$$

The coefficients of group parameters must vanish, giving relations between $\omega_{X, Y}$ and $v_{+}$and between $v_{+}$and $v_{-}$. Repeating the procedure starting with the qrelations between $v_{-}$and $a$ and $d_{\text {, we arrive at the general relations }}$

$$
\begin{aligned}
& w_{i} v_{ \pm}=v_{ \pm} \omega_{i} \quad i=1,2, X, Y \\
& v_{+} v_{-}=v_{-} v_{+} .
\end{aligned}
$$

Note that these relations are independent of $q$ and equivalent to the classical case $q=1$.

The procedure may now be repeated with the relations between $\omega_{Y}$ and $a$ and $d$. Applying $\delta$ gives

$$
\begin{aligned}
& \delta\left(\omega_{Y}-\omega_{Y} a\right)=\left(f\left(\omega_{X} \omega_{Y}+\omega_{Y} \omega_{X}\right)+2(1-\phi) \omega_{Y}^{2}\right) a=0 \\
& \delta\left(d_{Y}-\omega_{Y} d\right)=\left(f\left(\omega_{X} \omega_{Y}+\omega_{Y} \omega_{X}\right)-2(1+\phi) \omega_{Y}^{2}\right) d=0 .
\end{aligned}
$$

Vanishing requires

$$
\begin{aligned}
& \omega_{Y}^{2}=0 \\
& \omega_{X} \omega_{Y}+\omega_{Y} \omega_{X}=0 .
\end{aligned}
$$

Again we find relations independent of $q$ and equivalent to the classical case.

There is one more set of relations, involving $\omega_{X}$. Applying $\delta$ to the relations between $a$ and $\omega_{X}$ gives

$$
\delta\left(a \omega_{X}-f \omega_{X} a\right)=\left(f(f+1) \omega_{X}^{2}+\left(f-q^{2}\right) v_{+} v_{-}\right)=0 .
$$

This yields the relation

$$
\omega_{X}^{2}=\kappa v_{+} v_{-} \quad \kappa=\frac{q^{2}-f}{f(f+1)} .
$$


Notice that this relation is not equivalent to the classical case where $\omega_{X}^{2}=0$. It involves both the quantum deformation parameter $q$ and the undetermined parameter $f$. There is a final consistency check which will determine $f$. Using the q-relations between between group parameters and Cartan-Maurer forms, we find

$$
\begin{aligned}
A_{r}\left(\omega_{X}^{2}-\kappa v_{+} v_{-}\right) & =\left(f^{2} \omega_{X}^{2}-q^{2} \kappa v_{+} v_{-}\right) A_{r} \\
& =\left(f^{2}-q^{2}\right) \kappa v_{+} v_{-} A_{r} \\
& =\frac{\left(f^{2}-q^{2}\right)\left(q^{2}-f\right)}{f(f+1)} v_{+} v_{-} A_{r} \\
& =0 .
\end{aligned}
$$

There are three solutions for $f$ :

$$
\begin{aligned}
\text { I) } f=q^{2} & \kappa=0 \\
\text { II) } f=q & \kappa=\frac{q-1}{q+1} \\
\text { III) } f=-q & \kappa=\frac{q+1}{q-1} .
\end{aligned}
$$

The first solution is the simplest, with $\kappa=0$ and (3.15) equivalent to the classical case. The third solution has the wang limit of (3.12) for $q=1$ and we will disregard this solution.

With the q-relations just derived we may rewrite the Cartan-Maurer equations in a simplified form. They are

$$
\begin{aligned}
& \delta \omega_{Y}=0 \\
& \delta \omega_{X}=(\kappa-1) v_{+} v_{-} \\
& \delta v_{ \pm}= \pm 2 \omega_{y} v_{ \pm} .
\end{aligned}
$$

For solution $I$ we see once again that the equations agree with the classi-al case. This is not true for $\delta \omega_{X}$ for solution $I I$.

\subsection{The Algebra}

The reiations between Cartan-Miaurer forms provide enough information to determine the algebra of the quantum group. Using these relations to rearrange the 
expansion of $\delta^{2}(3.7)$ we get

$$
\begin{aligned}
\delta^{2}= & v_{+}^{2} \nabla_{+}^{2}+v_{-}^{2} \nabla_{-}^{2}+\omega_{X} \omega_{Y}(X Y-Y X) \\
& +V_{+} \omega_{Y}\left(Y \nabla_{+}-\nabla_{+} Y+2 \nabla_{+}\right)+V_{-} \omega_{Y}\left(Y \nabla_{-}-\nabla_{-} Y+2 \nabla_{-}\right) \\
& +v_{+} \omega_{X}\left(X \nabla_{+}-\nabla_{+} X\right)+v_{-} \omega_{X}\left(X \nabla_{-}-\nabla_{-} X\right) \\
& +v_{+} v_{-}\left(\nabla_{+} \nabla_{-}+\nabla_{-} \nabla_{+}+(\kappa-1) X-\kappa X^{2}\right)
\end{aligned}
$$

Nilpotency of $\delta$ requires the coefficients of the Cartan-Maurer forms to vanish:

$$
\begin{aligned}
& X Y-Y X=0 \\
& \nabla_{ \pm} Y-Y \nabla_{ \pm}= \pm 2 \nabla_{ \pm} \\
& \nabla_{ \pm}^{2}=0 \\
& \nabla_{ \pm} X-X \nabla_{ \pm}=0 \\
& \nabla_{+} \nabla_{-}+\nabla_{-} \nabla_{+}=(1-\kappa) X+\kappa X^{2} .
\end{aligned}
$$

This is the algebra of the generators of $\mathrm{GL}_{\mathbf{q}}(1 \mid 1)$.

There are several features to note about the algebra derived above. First, note that the generator $X$ commutes with all other generators. Thus it may be redefined by any function $X^{\prime}=F(X)$. Specifically it is desirable to simplify the algebra by setting the anticommutator $\left\{\nabla_{+}, \nabla_{-}\right\}_{+}$equal to the redefined generator $X^{\prime}$ :

$$
X^{\prime}=\nabla_{+} \nabla_{-}+\nabla_{-} \nabla_{+}= \begin{cases}X_{I} & \text { solution I } \\ \frac{2}{q+1} X_{I I}+\frac{q-1}{q+1} X_{I I}^{2} & \text { solution II . }\end{cases}
$$

For solution $I$ no redefintion is necessary. For solution $I I$ the above redefinition gives $X_{I}$ in terms of $X_{I I}$. Later we will see that this redefinition is consistent with the coproduct.

With this redefinition, or always for solution $I$, we see that the algebra is independent of $q$. In fact the algebra coincides with the classic’! algebra. Shortly we will see that this is not true for the coproduct, which does depend on $q$ and is not equivalent to the classical coproduct.

Finally, we note that the generators $\nabla_{ \pm}$and $X$ form a closed subalgebra, given by the last three lines of (3.18). Recall the quantum determinant defined in 


$$
D=\left(\alpha-\beta d^{-1} \gamma\right) d^{-1} .
$$

Using (3.3) for the action of the exterior derivative on the group parameters and $(3.10,3.12)$ to commute forms, one finds

$$
\delta D=2 \omega_{Y} D .
$$

From the expansion of $\delta(3.6)$, this shows that

$$
X D=0 \quad \nabla_{ \pm} D=0 .
$$

Thus we see that the generators in the subalgebra leave the determinant invariant. Then this subalgebra is $\mathrm{SL}_{\mathbf{q}}(1 \mid 1)$, the algebra of linear transformation with determinant one.

\subsection{The Coproduct}

Now we will consider the action of the generators on functions of the group parameters and on products of functions. This can be done using the previously derived q-relations between Cartan-Maurer forms and group parameters. This study yields the coproduct of the quantum algebra.

First recall the action of the exterior derivative on a product of group parameters $A_{r}, A, \in\{a, \beta, \gamma, d\}$ :

$$
\delta\left(A_{r} A_{s}\right)=\left(\delta A_{r}\right) A_{s}+(-1)^{A_{r}} A_{r}\left(\delta A_{s}\right) .
$$

Using the relations $(3.10,3.12)$, one can commute the Cartan-Maurer forms in the second term to the left of $A_{r}$. Equating coefficients of the 1-forms on both sides of the equation gives

$$
\begin{aligned}
X\left(A_{r} A_{s}\right) & =\left(X A_{r}\right) A_{\mathbf{s}}+f A_{r}\left(X A_{\mathbf{s}}\right) \\
Y\left(A_{r} A_{\mathbf{s}}\right) & =\left(Y A_{r}\right) A_{\mathbf{s}}+A_{r}\left(Y A_{s}\right) \\
\nabla_{ \pm}\left(A_{r} A_{s}\right) & =\left(\nabla_{ \pm} A_{r}\right) A_{\mathbf{s}}+(-1)^{A_{r}} q A_{r}\left(\nabla_{ \pm} A_{\mathbf{s}}\right)
\end{aligned}
$$

where for now we will consider both solutions $f=q, q^{2}$. Repeating this procedure for higher power monomials of the group parameters will determine the action 
on arbitrary functions. It is convenient to simplify this notation by dropping the second term in the product ( $A_{s}$ here) and writing the action of the the generators on $A_{r}$ times an arbitrary function $(\cdot)$ to follow:

$$
\begin{aligned}
X A_{r^{\prime}} & =\left(X A_{r}\right)+f A_{r} X . \\
Y A_{r^{\prime}} & =\left(Y A_{r}\right) \cdot+A_{r} Y . \\
\nabla_{ \pm} A_{r^{\prime}} & =\left(\nabla_{ \pm} A_{r}\right) \cdot+(-1)^{A_{r}} q A_{r} \nabla_{ \pm} \cdot
\end{aligned}
$$

We consider in detail first the generator $X$. Consider a monomial of group parameters $a^{m} d^{m} \beta^{i} \gamma^{j}$ where $m, n \geq 0$ and $i, j=0,1$. From above we see that a power of $f$ appears each time we commute $X$ through a group parameter. Repeating the procedure gives

$$
\begin{aligned}
X\left(a^{m} d^{n} \beta^{i} \gamma^{j}\right) & =\left(1+f+f^{2}+\ldots+f^{m+n+i+j}\right)\left(a^{m} d^{m} \beta^{i} \gamma^{j}\right) \\
& =\frac{f^{m+n+i+j}-1}{f-1} a^{m} d^{m} \beta^{i} \gamma^{j} .
\end{aligned}
$$

It is convenent to define a number operator $N$ for the group parameters as follows:

$$
\begin{aligned}
& \left(N A_{r}\right)=A_{r} \\
& N A_{r}=\left(N A_{r}\right) \cdot+A_{r} N \\
& \left(N a^{m} d^{m} \beta^{i} \gamma^{j}\right)=(m+n+i+j)\left(a^{m} d^{m} \beta^{i} \cdot \gamma^{j}\right) .
\end{aligned}
$$

Then the generator $X$ and $N$ are related by

$$
\begin{aligned}
X & =\frac{f^{N}-1}{f-1} \\
f^{N} & =1+(f-1) X .
\end{aligned}
$$

Now any funtion $F$ of the group parameters can be written as a sum of monomials. By repeating the above procedures on a product of monomials, we find the action of $X$ on products of functions to be

$$
\begin{aligned}
X F \cdot & =(X F) \cdot+\left(f^{N} F\right) X . \\
& =(X F) \cdot+((1+(f-1) X) F) X .
\end{aligned}
$$

The result for the generators $\nabla_{ \pm}$is obtained in a similar manner. Here instead of a factor of $f$ (which is equal to $q$ or $q^{2}$ ) there is always a factor of $q$ 
when commuting through a group parameter. On a product of functions

$$
\nabla_{ \pm} F \cdot=\left(\nabla_{ \pm} F\right) \cdot+(-1)^{F}\left(q^{N} F\right) \nabla_{ \pm} \cdot
$$

Finally for the generator $Y$ we obtain the simple result

$$
Y F \cdot=(Y F)+F Y
$$

In this final case note that there are no factors of $q$. In fact the action of $Y$ is equivalent to the classical case.

These results lead to the coproduct of the quantum algebra. If $A$ is the universal enveloping algebra, then the coproduct $\Delta$ is a map to the tensor product of $\mathcal{A}$ :

$$
\begin{aligned}
\Delta & : \mathcal{A} \\
& \rightarrow \mathcal{A} \otimes \mathcal{A} \\
& \mapsto \Delta(F) \quad F \in \mathcal{A} .
\end{aligned}
$$

The map must be a homomorphism of the algebra:

$$
\Delta(F G)=\Delta(F) \Delta(G) \quad F, G \in \mathcal{A} .
$$

It is straightforward to read off the coproduct from the results in equations (3.1S3.21). For both solutions we have

$$
\begin{aligned}
\Delta\left(\nabla_{ \pm}\right) & =\nabla_{ \pm} \otimes 1+q^{N} \otimes \nabla_{ \pm} \\
\therefore Y) & =Y \otimes 1+1 \otimes Y \\
\Delta\left(X_{I}\right) & =X_{I} \otimes 1+q^{2 N} \otimes X_{I} \\
\Delta\left(X_{I I}\right) & =X_{I I} \otimes 1+q^{N} \otimes X
\end{aligned}
$$

where in terms of $X$

$$
q^{N}= \begin{cases}\left(1+\left(q^{2}-1\right) X_{I}\right)^{\frac{1}{2}} & \text { solution } I \\ 1+(q-1) X_{I I} & \text { solution } I I .\end{cases}
$$

Finally, we note that the redefinition of $X$ for the two solutions for $f$ :

$$
X_{I}=\frac{2}{q+1} X_{I I}+\frac{q-1}{q+1} X_{I I}^{2}
$$

is consisient with the coproduct:

$$
\Delta\left(X_{I}\right)=\frac{2}{q+1} \Delta\left(X_{I I}\right)+\frac{q-1}{q+1} \Delta\left(X_{I I}\right)^{2} .
$$


This shows that both solutions for $X$ are in the same universal enveloping algebra, and are in fact reparameterizations of each other. For the remainder of the discussion we will take solution $I$ with $f=q^{2}$ and set $X=X_{I}$.

\subsection{Method of Faddeev, Reshetikhin, and Takhtajan}

It is instructive to compare the results based on the differential calculus to thcse of Faddeev, Reshetikhin, and Takhtajan [6,7]. Their method is based on the $P$ matrix (2.4) used earlier in defining the quantum group. Define the matrix $E_{i}^{\prime}$ by

$$
R^{\prime}=P R P=\left(\begin{array}{cccc}
q & 0 & 0 & 0 \\
0 & 1 & \lambda & 0 \\
0 & 0 & 1 & 0 \\
0 & 0 & 0 & q^{-1}
\end{array}\right)
$$

Here $P$ is the permutation matrix acting on the tensor product of vector spaces $\mathcal{V} \otimes \mathcal{V}$ according to $P(u \otimes v)=v \otimes u$ for $u, v \in \mathcal{V}$, or:

$$
P=\left(\begin{array}{llll}
1 & 0 & 0 & 0 \\
0 & 0 & 1 & 0 \\
0 & 1 & 0 & 0 \\
0 & 0 & 0 & 1
\end{array}\right)
$$

Recall also that $\lambda=q-q^{-1}$. Next define the upper-and lower-triangular $2 \times 2$ matrices

$$
L^{+}=\left(\begin{array}{cc}
U_{+} & \lambda \chi_{+} \\
0 & W_{+}
\end{array}\right) \quad L^{-}=\left(\begin{array}{cc}
U_{-} & 0 \\
-\lambda \chi_{-} & W_{-}
\end{array}\right) .
$$


Finally define the $4 \times 4$ matrices $L_{1,2}$ which act on $\mathcal{V} \otimes \mathcal{V}$ by $L_{1}^{ \pm}=L^{ \pm} \otimes 1$ and $L_{2}^{ \pm}=1 \otimes L^{ \pm}$. These matrices are

$$
\begin{aligned}
L_{1}^{+}=\left(\begin{array}{cccc}
U_{+} & 0 & \lambda \chi_{+} & 0 \\
0 & U_{+} & 0 & \lambda \chi_{+} \\
0 & 0 & W_{+} & 0 \\
0 & 0 & 0 & W_{+}
\end{array}\right) & L_{1}^{-}=\left(\begin{array}{cccc}
U_{-} & 0 & 0 & 0 \\
0 & U_{-} & 0 & 0 \\
-\lambda \chi_{-} & 0 & W_{-} & 0 \\
0 & -\lambda \chi_{-} & 0 & W_{-}
\end{array}\right) \\
L_{2}^{+}=\left(\begin{array}{cccc}
U_{+} & \lambda \chi_{+} & 0 & 0 \\
0 & W_{+} & 0 & 0 \\
0 & 0 & U_{+} & -\lambda \chi_{+} \\
0 & 0 & 0 & W_{+}
\end{array}\right) & L_{2}^{-}=\left(\begin{array}{cccc}
U_{-} & 0 & 0 & 0 \\
-\lambda \chi_{-} & W_{-} & 0 & 0 \\
0 & 0 & U_{-} & 0 \\
0 & 0 & \lambda \chi_{-} & W_{-}
\end{array}\right) .
\end{aligned}
$$

Here we have used index ordering to allow simple matrix multiplication (see appendix).

The method of Faddeev, Reshetikhin, and Takhtajan uses these matrices to determine the quantum algebra and coproduct. The equations

$$
\begin{aligned}
& R^{\prime} L_{1}^{ \pm} L_{2}^{ \pm}=L_{2}^{ \pm} L_{1}^{ \pm} R^{\prime} \\
& R^{\prime} L_{1}^{+} L_{2}^{-}=L_{2}^{-} L_{1}^{+} R^{\prime}
\end{aligned}
$$

yield relations on $U_{ \pm}, W_{ \pm}$, and $\chi_{ \pm}$. These relations lead to the quantum algebra. One finds that $U_{ \pm}$and $W_{ \pm}$all commute with each other, and also that the combinations $U_{+} U_{-}$and $W_{+} W_{-}$commute with $\chi_{ \pm}$. Then we may take these combinations to be proportional to the identity and define

$$
\begin{array}{ll}
U_{+}=U & U_{-}=U^{-1} \\
W_{+}=W & W_{-}=W^{-1} .
\end{array}
$$

Then the remaining relations are

$$
\begin{aligned}
& \chi_{ \pm} U=q^{ \pm 1} U \chi_{ \pm} \\
& \chi_{ \pm} W=q^{ \pm 1} W \chi_{ \pm} \\
& \chi_{+} \chi-+\chi-\chi_{+}=\lambda^{-1}\left(U^{-1} W-U W^{-1}\right)
\end{aligned}
$$

These relations determine the quantum algebra. 
The coproduct is determined by

$$
\Delta\left(L^{ \pm}\right)=L^{ \pm} \dot{\otimes} L^{ \pm}
$$

where $\dot{\otimes}$ denotes tensor product combined with usual matrix multiplication. Using the matrices $L^{ \pm}$defined above one gets

$$
\begin{aligned}
\Delta(U) & =U \otimes U \quad \Delta(W)=W \otimes W \\
\Delta\left(\chi_{+}\right) & =\chi_{+} \otimes W+U \otimes \chi_{+} \\
\Delta\left(\chi_{-}\right) & =\chi_{-} \otimes U^{-1}+W^{-1} \otimes \chi_{-}
\end{aligned}
$$

where we have used the above definitions of $U$ and $W$.

These results may now be compared to the algebra and coproduct derived earlier using the differential calculus. We can do this by writing one set of generators as functions of the other set. Because of the rather simple form of the algebra, there are many such reparameterizations which yield the same form of the algebra. However, the coproduct will give a unique relation between the two formulations. Consider the combinations $W^{-1} \chi_{+}$and $U_{\chi}$. Using the coproducts (3.24) we find

$$
\begin{gathered}
\Delta\left(W^{-1} \chi_{+}\right)=W^{-1} \chi_{+} \otimes 1+U W^{-1} \otimes W^{-1} \chi_{+} \\
\Delta\left(U \chi_{-}\right)=U \chi_{-} \otimes 1+U W^{-1} \otimes U \chi_{-} .
\end{gathered}
$$

Comparison with the coproducts (3.22) indicates that $W^{-1} \chi_{+} \propto \nabla_{+}$and $U_{\chi} \propto$ $\nabla_{\text {. }}$. Including normalization factors to get the same algebra, we find that the first set of generators can be written in terms of the new ones as

$$
\begin{array}{ll}
\nabla_{+}=q^{-1} W^{-1} \chi_{+} & X=\frac{U^{2} W^{-2}-1}{q^{2}-1} \\
\nabla_{-}=q^{-1} U \chi_{-} & q^{N}=U W^{-1} .
\end{array}
$$

For the inverse relations, note that

$$
q^{-\frac{1}{2} Y} \nabla_{ \pm}=q^{\mp 1} \nabla_{ \pm} q^{-\frac{1}{2} Y}
$$

where we have used the commutation relations for $Y$ in (3.18). Then it is easy to see that

$$
\begin{array}{ll}
\chi_{+}=q q^{-\frac{1}{2} Y-\frac{1}{2} N} \nabla_{+} \quad & U=q^{-\frac{1}{2} Y+\frac{1}{2} N} \\
\chi_{-}=q q^{\frac{1}{2} Y-\frac{1}{2} N} \nabla_{-} & W=q^{-\frac{1}{2} Y-\frac{1}{2} N}
\end{array}
$$


are the correct reparameterizations. Sulstitution of these relations into one form of the algebra leads to the other form. Thus both methods yield the same quantum universal enveloping algebra. 


\section{Chapter 4}

\section{The Quantum Super-Plane}

In this chapter we discuss the quantum super-plane. This follows an approach to quantum groups first suggested by Y. Kobyzev and developed by Manin [5]. By defining coordinates and differentials which obey q-relations (i.e. they do not obey classical commutations rules), and demanding covariance, one obtains the previous q-relations on the elements of matrices which act on the plane coordinates. The converse is also true: the q-relations on the matrix e'ements imply the q-relations on coordinates and differentials.

We will follow this approach for the quantum group $\mathrm{GL}_{\mathrm{q}}(1 \mid 1)$. First we will show the equivalence of group q-relations and super-plane q-relations. Then we will develop a covariant differential calculus on the super-plane. Finally, we obtain a representation of the generators of $\mathrm{GL}_{\mathbf{q}}(1 / 1)$ in terms of the super-plane coordinates.

\subsection{Coordinate and Differential q-relations}

We begin by defining the super-plane coordinate vector $V$ and differential vector $U$ with components

$$
V=\left(\begin{array}{l}
x \\
\xi
\end{array}\right) \quad U=\left(\begin{array}{l}
\eta \\
y
\end{array}\right) .
$$


The corresponding components of $V$ and $U$ have opposite Grassmann parity. Following Manin we impose the q-relations

$$
\begin{array}{ll}
x \xi=q \xi x & \xi^{2}=0 \\
y \eta=q \eta y & \eta^{2}=0 .
\end{array}
$$

(The nilpotency conditions on $\xi$ and $\eta$ are the same as in the classical case.) These vectors are acted on by matrices $A$ with elements

$$
A=\left(\begin{array}{ll}
\alpha & \beta \\
\gamma & d
\end{array}\right)
$$

where we do not yet know the commutation relations between $a, \beta, \gamma$, and $d$. The matrix elements all commute or anticommute with the vector components, depending on Grassmann parity. The vectors transform under left-multiplication by $A$ :

$$
V^{\prime}=A V \quad U^{\prime}=A U
$$

with new components

$$
\begin{array}{ll}
x^{\prime}=a x+\beta \xi & \eta^{\prime}=a \eta+\beta y \\
\xi^{\prime}=\gamma x+d \xi & y^{\prime}=\gamma \eta+d y .
\end{array}
$$

We now impose the condition that the q-relations (4.2) are covariant, i.e. that the transformed components satisfy the same q-relations. This leads to the familiar q-relations on the matrix elements. For example, the products

$$
\begin{aligned}
& x^{\prime} \xi^{\prime}=a \gamma x^{2}+\left(a d-q^{-1} \beta \gamma\right) x \xi \\
& \xi^{\prime} x^{\prime}=\gamma a x^{2}+\left(\gamma \beta-q^{-1} d a\right) x \xi
\end{aligned}
$$

can be plugged into $x^{\prime} \xi^{\prime}=q \xi^{\prime} x^{\prime}$. Equating coefficients of $x^{2}$ and $x \xi$ gives

$$
a \gamma=q \gamma a \quad a d-q^{-1} \gamma \beta=q \gamma \beta+d a .
$$

Summarizing, the super-plane relations and the matrix element relations they imply are

$$
\begin{array}{rlll}
x^{\prime} \xi^{\prime}=q \xi^{\prime} x^{\prime} & \Longrightarrow & a \gamma=q \gamma a & a d-q^{-1} \beta \gamma=d a+q \gamma \beta \\
y^{\prime} \eta^{\prime}=q \eta^{\prime} y^{\prime} & \Rightarrow & d \beta=q \beta d & a d+q \beta \gamma=d a-q^{-1} \gamma \beta \\
\xi^{\prime 2}=0 & \Rightarrow & d \gamma=q \gamma d & \gamma^{2}=0 \\
\eta^{2}=0 & \Longrightarrow & a \beta=q \beta a & \beta^{2}=0 .
\end{array}
$$


Finally, the last equations in the first two lines imply

$$
a d-d a=-\left(q-q^{-1}\right) \beta \gamma \quad \beta \gamma=-\gamma \beta
$$

and we see that we have reproduced the q-relations between group parameters (2.5) as defined by the R-matrix method.

The converse of these arguments is also true. Given the q-relations between group parameters, one can find bilinear q-relations on the super-plane coordiantes and differentials which are preserved by a group rotation. This means that if the bilinear relations are given by

$$
F_{i}(x, \xi, \eta, y)=0
$$

then the transformed relations also vanish:

$$
F_{i}\left(x^{\prime}, \xi^{\prime}, \eta^{\prime}, y^{\prime}\right)=0
$$

where the primed components are given by (4.4). In fact it will be seen that this final function is a linear combination of the previous ones:

$$
F_{i}\left(x^{\prime}, \xi^{\prime}, \eta^{\prime}, y^{\prime}\right)=\sum_{j} r_{i j}(a, \beta, \gamma, d) F_{j}(x, \xi, \eta, y) .
$$

Such a set of preserved relations is said to be covariant under the quantum group action.

It is easy to check these relations for the quantum super-plane, since we already know what they should be. However it is instructive to examine at least one case in detail since these techniques will be used later. Let us consider bilinears in the coordinates $x$ and $\xi$. Using the primed components (4.4) and the q-relations on group parameters to rearrange terms we get for $x$ and $\xi$

$$
\begin{aligned}
x^{\prime} \xi^{\prime} & =\gamma a q x^{2}+a d x \xi-\beta \gamma \xi x+\beta d \xi^{2} \\
\xi^{\prime} x^{\prime} & =\gamma a x^{2}+a d \xi x+\beta \gamma(\lambda \xi x-x \xi)-\beta d q \xi^{2} \\
\xi^{\prime} & =\gamma d(x \xi-q \xi x)+d^{2} \xi^{2}
\end{aligned}
$$

and it is easily seen that covariant combinations are

$$
\begin{aligned}
x^{\prime} \xi^{\prime}-q \xi^{\prime} x^{\prime} & =(a d-\beta \gamma)(x \xi-q \xi x)+\beta d\left(1+q^{2}\right) \xi^{2} \\
\xi^{\prime 2} & =\gamma d(x \xi-q \xi x)+d^{2} \xi^{2} .
\end{aligned}
$$


Proceeding similarly with the differentials shows that

$$
\begin{aligned}
y^{\prime} \eta^{\prime}-q \eta^{\prime} y^{\prime} & =\left(a d-q^{-1} \beta y\right)(y \eta-q \eta y)+\gamma a\left(1+q^{2}\right) \eta^{2} \\
\eta^{2} & =a^{2} \eta^{2}+\beta a(y \eta-q \eta y) .
\end{aligned}
$$

are covariant. Summarizing, we may write these covariant combinations as

$$
\begin{array}{ll}
F_{1}=x \xi-q \xi x=0 & F_{2}=\xi^{2}=0 \\
F_{3}=y \eta-q \eta y=0 & F_{4}=\eta^{2}=0 .
\end{array}
$$

We have recovered the relations with which we began this discussion, and we see that the arrows in (4.5) run in both directions.

Finally, we note that the q-relations on cocrdinates and differentials can be derived from the R-matrix. Define two column vectors in the tensor product of the vector space acted on by the quantum matrices:

$$
\left(V_{1} \otimes V_{2}\right)=\left(\begin{array}{c}
x^{2} \\
x \xi \\
\xi x \\
\xi^{2}
\end{array}\right) \quad\left(U_{1} \otimes U_{2}\right)=\left(\begin{array}{c}
\eta^{2} \\
-\eta y \\
y \eta \\
-y^{2}
\end{array}\right) .
$$

(The signs in $\left(U_{1} \otimes U_{2}\right)$ are needed for covariance, as discussed for the vector $U$ in the next section.) Then using $R$ from (2.4) and $P$ from the previous chapter define the matrix $\hat{R}$ :

$$
\hat{R}=P R=\left(\begin{array}{cccc}
q & 0 & 0 & 0 \\
0 & \lambda & 1 & 0 \\
0 & 1 & 0 & 0 \\
0 & 0 & 0 & -q^{-1}
\end{array}\right)
$$

where $\lambda=q-q^{-1}$. The the equations

$$
(\hat{R}-q 1)\left(V_{1} \otimes V_{2}\right)=0 \quad\left(\hat{R}+q^{-1} 1\right)\left(U_{1} \otimes U_{2}\right)=0
$$

determine the coordinate and differential q-relations (4.2).

\subsection{Super-Plane Exterior Derivative}

Next we wish to formulate a covariant differential calculus on the quantum superplane, involving the derivatives of the coordinates and their differentials. As with 
the quantum algebra, ve begin by defining an exterior derivative $\delta$ which is nilpotent and obeys the graded Leibniz rule (3.1). Expand $\delta$ in products of differentials with derivatives:

$$
\delta=\delta x \partial_{x}+\delta \xi \partial_{\xi}
$$

Here $\delta x$ and $\partial_{\xi}$ are Grassmann odd and $\delta \xi$ and $\partial_{x}$ are Grassmann even so that overall $\delta$ is odd. Comparing to the differential vector $U$ in (4.1), one might expect to identify $\delta x=\eta$ and $\delta \xi=y$. However, this is not quite correct. We wish a calculus which is covariant under the group action defined in (4.3). Applying $\delta$ to the transformed components of $V$ gives

$$
\begin{aligned}
& \delta x^{\prime}=a \delta x-\beta \delta \xi \\
& \delta \xi^{\prime}=-\gamma \delta x+d \delta \xi .
\end{aligned}
$$

Now if we define

$$
\eta=\delta x \quad y=-\delta \xi
$$

as the components of $U$, we find that this differential is also covariant under the group action (4.3).

The exterior derivative is now written

$$
\delta=\eta \partial_{x}-y \partial_{\xi}
$$

As with the algebra, the general properties of $\delta$ allow one to determine some information on the algebra of the derivatives. Checking nilpotency gives

$$
\begin{aligned}
\delta^{2} & =-\eta\left(\eta \partial_{x}-y \partial_{\xi}\right) \partial_{x}-y\left(\eta \partial_{x}-y \partial_{\xi}\right) \partial_{\xi} \\
& =\eta y\left(\partial_{\xi} \partial_{x}-q \partial_{x} \partial_{\xi}\right)+y^{2} \partial_{\xi}^{2} \\
& =0
\end{aligned}
$$

where we have used (4.2). The coefficients of the forms must vanish, leaving

$$
\partial_{\xi} \partial_{x}=q \partial_{x} \partial_{\xi} \quad \partial_{\xi}^{2}=0
$$

as the basic algebra of the derivatives. 


\subsection{Additional q-relations}

We wish to extend the derivative algebra to include the coordinates $x$ and $\xi$. As with the group algebra, we will use $\delta$ and the Leibniz rule to determine this. This will require that we know how to commute differentials with coordinates. Some information can be obtained from the basic coordinate q-relations in (4.6). Applying $\delta$ gives

$$
\begin{aligned}
\delta(x \xi-q \xi x) & =\eta \xi-x y+q y x+q \xi \eta=0 \\
\delta\left(\xi^{2}\right) & =-y \xi+\xi y=0 .
\end{aligned}
$$

The second relation tells us how to commute $y$ and $\xi$. However, the first relation does not provide all the information needed for the rest of the components. More assumptions will be needed for these relations.

The first line of (4.8) indicates that there may be some mixing between the coordinate-differential bilinears. Suppose that one relation takes the form

$$
x y=r y x+r^{\prime} \eta \xi .
$$

One of the parameters may be eliminated by requiring that $\delta$ applied to this relation be consistent with $y \eta=q \eta y$. This fixes $r^{\prime}=1-q r$. Finally, adding a multiple of $\delta F_{1}$ gives a similar relation for $\xi$ and $\eta$, leaving

$$
\begin{aligned}
& x y=r y x+(1-q r) \eta \xi \\
& \xi \eta=-r \eta \xi+\left(q^{-1} r-1\right) y x .
\end{aligned}
$$

The parameter $r$ may be determined by a consistency check. Using the second relation twice to commute $\eta$ through $\xi^{2}$ and recalling that $\xi^{2}=0$ gives

$$
\xi^{2} \eta=\left(1-q^{-1} r\right)(q r-1) y \xi x=0
$$

which is solved by $r=q^{ \pm 1}$. Plugging into the above relations we have for $r=q$

$$
\begin{aligned}
& x y=q y x+\left(1-q^{2}\right) \eta \xi \\
& \xi \eta=-q \eta \xi
\end{aligned}
$$

and for $r=q^{-1}$

$$
\begin{aligned}
& x y=q^{-1} y x \\
& \xi \eta \eta=-q^{-1} \eta \underline{\xi}+\left(q^{-2}-1\right) y x .
\end{aligned}
$$


The relations between these bilinears have been completely determined.

Relations between $x$ and $\eta$ are still needed. To determine these recall the covariance of $F_{1-4}$ in (4.6) under the group action as discussed earlier. Requiring covariance for the new relations will give the missing relations. First, for $r=q$ consider

$$
\begin{aligned}
\xi^{\prime} \eta^{\prime}+q \eta^{\prime} \xi^{\prime}= & a d(\xi \eta+q \eta \xi)+\beta d q(y \xi-\xi y) \\
& \quad+\gamma \beta\left(x y-q y x+\left(q^{2}-1\right) \eta \xi\right)+\gamma \alpha\left(x \eta-q^{2} \eta x\right) \\
= & 0
\end{aligned}
$$

This vanishes if the new relations are valid and if $x \eta=q^{2} \eta x$. For $r=q^{-1}$ take

$$
\begin{aligned}
x^{\prime} y^{\prime}-q^{-1} y^{\prime} x^{\prime}= & a d\left(x y-q^{-1} y x\right)+\beta d(\xi y-y \xi) \\
& +\gamma \beta\left(\xi \eta+q^{-1} \eta \xi+\left(1-q^{-2}\right) y x\right)+\gamma a\left(q x \eta-q^{-1} \eta x\right) \\
= & 0 .
\end{aligned}
$$

which will vanish if $x \eta=q^{-2} \eta x$. All of the needed relations have been determined. Summarizing, we have for $r=q$ :

$$
\begin{array}{ll}
x y=q y x+\left(1-q^{2}\right) \eta \xi & x \eta=q^{2} \eta x \\
\xi \eta=-q \eta \xi & \xi y=y \xi
\end{array}
$$

and for $r=q^{-1}$ :

$$
\begin{array}{ll}
x y=q^{-1} y x & x \eta=q^{-2} \eta x \\
\xi \eta=-q^{-1} \eta \xi+\left(q^{-2}-1\right) y x & \xi y=y \xi .
\end{array}
$$

Finally, although we have only shown covariance for some of the relations, it can be shown that for each case $r=q^{ \pm 1}$ all of the relations listed here are covariant.

\subsection{Derivative q-relations}

Now we can determine the operator relations between derivatives and coordinates. We will use our previous notation for operator equations where arbitrary functions 
to the right are indicated by $(\cdot)$. Applying $\delta$ to $x \cdot$ gives

$$
\begin{aligned}
\delta x \cdot & =\eta \partial_{x} x \cdot-y \partial_{\xi} x . \\
& =(\delta x) \cdot+x \delta \cdot \\
& =\eta \cdot+x \eta \partial_{x} \cdot-x y \partial_{\xi} .
\end{aligned}
$$

where the second line is a result of the Leibniz rule for $\delta$. Using the q-relations $(4.9,4.10)$ we can commute the differentials to the left in the third line. Comparing to the first line gives the desired derivative operator relations. Similarly, for $\xi$. we have

$$
\begin{aligned}
\delta \xi \cdot & =\eta \partial_{x} \xi \cdot-y \partial_{\xi} \xi \\
& =(\delta \xi) \cdot-\xi \delta . \\
& =-y \cdot-\xi \eta \partial_{x} \cdot+\xi y \partial_{\xi} \cdot
\end{aligned}
$$

Now using the q-relations for $r=q$ we find

$$
\begin{array}{ll}
\partial_{x} x \cdot=1 \cdot+q^{2} x \partial_{x} \cdot+\left(q^{2}-1\right) \xi \partial_{\xi} \cdot & \partial_{x} \xi=q \xi \partial_{x} \cdot \\
\partial_{\xi} \xi=1 \cdot-\xi \partial_{\xi} \cdot & \partial_{\xi} x=q x \partial_{\xi}
\end{array}
$$

and for $r=q^{-1}$

$$
\begin{array}{ll}
\partial_{x} x \cdot=1 \cdot+q^{-2} x \partial_{x} \cdot & \partial_{x} \xi \cdot=q^{-1} \xi \partial_{x} \\
\partial_{\xi} \xi=1 \cdot-\xi \partial_{\xi} \cdot+\left(q^{-2}-1\right) x \partial_{x} \cdot & \partial_{\xi} x \cdot=q^{-1} x \partial_{\xi} \cdot
\end{array}
$$

These two sets of relations are the desired derivative operator equations.

There are also similar operator relations between the derivatives and the differentials. They are obtained by acting with the derivatives on the q-relations between differentials and coordinates. For example, for $r=q$ one finds

$$
\begin{array}{ll}
\partial_{x}(\eta x)=q^{-2} \eta & \partial_{\xi}(\eta x)=0 \\
\partial_{x}(\eta \xi)=0 & \partial_{\zeta}(\eta \xi)=-q^{-1} \eta \\
\partial_{x}(y x)=q^{-1} y & \partial_{\xi}(y x)=\left(q^{-2}-1\right) \eta \\
\partial_{x}(y \xi)=0 & \partial_{\xi}(y \xi)=y .
\end{array}
$$

Then these imply the operator relations

$$
\begin{array}{ll}
\partial_{x} \eta \cdot=q^{-2} \eta \partial_{x} \cdot & \partial_{\xi} \eta \cdot=-q^{-1} \eta \partial_{\xi} . \\
\partial_{x} y \cdot=q^{-1} y \partial_{x} \cdot & \partial_{\xi} y \cdot=y \partial_{\xi} \cdot+\left(q^{-2}-1\right) \eta \partial_{x} \cdot .
\end{array}
$$


Repeating the procedure for $r=q^{-1}$ gives

$$
\begin{array}{ll}
\partial_{x} \eta \cdot=q^{2} \eta \partial_{x}-\left(q^{2}-1\right) y \partial_{\xi} \cdot & \partial_{\xi} \eta \cdot=-q \eta \partial_{\xi} . \\
\partial_{x} y \cdot=q y \partial_{x} \cdot & \partial_{\xi} y=y \partial_{\xi} \cdot
\end{array}
$$

At this point we have determined all possible operator relations between the coordinates, differentials, and derivatives.

\subsection{Representation of Derivatives}

Next the derivative-coordinate operator relations $(4.11,4.12)$ can be used repeatedly to find similar relations between the derivatives and monomials in the coodinates. Then an arbitrary function can be written as a power series in the monomials. In fact, since $\xi^{2}=0$, the most general function in $x$ and $\xi$ can be written

$$
F(x, \xi)=F_{0}(x)+\xi F_{1}(x)
$$

and if $F(x, \xi)$ has definite Grassmann parity then $(-1)^{F}=(-1)^{F_{0}}=-(-1)^{F_{1}}$.

We take first the solution $r=q$. Using the operator relations (4.11) on a power of $x$ gives

$$
\begin{aligned}
\partial_{x} x^{n} \cdot & =\sum_{l=0}^{n-1} q^{2 l} x^{n-1}\left(1+\left(q^{2}-1\right) \xi \partial_{\xi}\right) \cdot+q^{2 n} x^{n} \partial_{x} . \\
& =x^{-1} \frac{q^{2 n}-1}{q^{2}-1} x^{n}\left(1+\left(q^{-2}-1\right) \xi \partial_{\xi}\right) \cdot+q^{2 n} x^{n} \partial_{x} \cdot
\end{aligned}
$$

Then for an arbitrary function of $x$ we have

$$
\partial_{x} F_{0}(x) \cdot=x^{-1} \frac{F_{0}\left(q^{2} x\right)-F_{0}(x)}{q^{2}-1}\left(1+\left(q^{2}-1\right) \xi \partial_{\xi}\right) \cdot+F_{0}\left(q^{2} x\right) \partial_{x} \cdot
$$

It is easy to extend this to a function multiplying $\xi$ :

$$
\begin{aligned}
\partial_{x} \xi F_{1}(x) \cdot & =q \xi \partial_{x} F_{1}(x) . \\
& =x^{-1} q^{2} \xi \frac{F_{1}\left(q^{2} x\right)-F_{1}(x)}{q^{2}-1}\left(1+\left(q^{2}-1\right) \xi \partial_{\xi}\right)+q \xi F_{1}\left(q^{2} x\right) \partial_{x} \cdot .
\end{aligned}
$$

Comparing the results for these two cases shows that for an arbitrary function $F(x, \xi)$

$$
\partial_{x} F(x, \xi) \cdot=x^{-1} \frac{F\left(q^{2} x, q^{2} \xi\right)-F\left(x, q^{2} \xi\right)}{q^{2}-1}\left(1+\left(q^{2}-1\right) \xi \partial_{\xi}\right)+F\left(q^{2} x, q \xi\right) \partial_{x} \cdot
$$


The results for $\partial_{\xi}$ are somewhat simpler. Repeating the above arguments we find

$$
\begin{aligned}
\partial_{\xi} x^{n} \cdot & =q^{n} x^{n} \partial_{\xi} \\
\partial_{\xi} F_{0}(x) \cdot & =(-1)^{F_{0}} F_{0}(q x) \partial_{\xi} . \\
\partial_{\xi} \xi F_{1}(x) \cdot & =F_{1}(x)-(-1)^{F_{1}} \xi F_{1}(q x) \partial_{\xi} \cdot
\end{aligned}
$$

Then for a general function as in (4.15)

$$
\partial_{\xi} F(x, \xi) \cdot=F_{1}(x) \cdot+(-1)^{F} F(q x, \xi) \partial_{\xi} .
$$

is the operator equation for $\partial_{\xi}$.

If there are no functions to the right of $F$ in the operator equations (4.16) and (4.17), $i$ se equations give the action of $\partial$ on $F$. We find for $F=F_{0}+\xi F_{1}$ as above

$$
\begin{aligned}
& \partial_{x} F(x, \xi)=x^{-2} \frac{F\left(q^{2} x, q^{2} \xi\right)-F\left(x, q^{2} \xi\right)}{q^{2}-1} \\
& \partial_{\xi} F(x, \xi)=F_{1}(x) .
\end{aligned}
$$

The operator equations may be rewritten in terms of these actions as

$$
\begin{aligned}
& \partial_{x} F(x, \xi) \cdot=\left(\partial_{x} F(x, \xi)\right)\left(1+\left(q^{2}-1\right) \xi \partial_{\xi}\right) \cdot+F\left(q^{2} x, q \xi\right) \partial_{x} \cdot \\
& \partial_{\xi} F(x, \xi) \cdot=\left(\partial_{\xi} F(x, \xi)\right) \cdot+(-1)^{F} F(q x, \xi) \partial_{\xi} \cdot
\end{aligned}
$$

This form of the equations is suggestive of a coproduct, for it gives the action of the derivative on a product of functions. We will dicuss this shortly.

The entire procedure may be repeated for the solution $r=q^{-1}$. The action of the derivatives is

$$
\begin{aligned}
& \partial_{x} F(x, \xi)=x^{-1} \frac{F\left(q^{-2} x, \xi\right)-F(x, \xi)}{q^{-2}-1} \\
& \partial_{\xi} F(x, \xi)=F_{1}\left(q^{-2} x\right)
\end{aligned}
$$

and the perator equations are

$$
\begin{aligned}
& \partial_{x} F(x, \xi) \cdot=\left(\partial_{x} F(x, \xi)\right) \cdot+F\left(q^{-2} x, q^{-1} \xi\right) \partial_{x} \cdot \\
& \partial_{\xi} F(x, \xi)=\left(\partial_{\xi} F(x, \xi)\right)\left(1+\left(q^{-2}-1\right) x \partial_{x}\right) \cdot+(-1)^{F} F\left(q^{-1} x, \xi\right) \partial_{\xi} \cdot
\end{aligned}
$$

We see that the mixing of derivatives is reversed between the two solutions, and $q$ is replaced by $q^{-1}$. 
It is useful to introduce number operators for the coordinates $x$ and $\xi$. They will allow the derivatives to be written in an illuminating operator form. Shortly they will also be used to write a representation of the algebra $\operatorname{GL}_{\mathbf{q}}(1 \mid 1)$. Define the number operators action on coordinates as

$$
\begin{array}{ll}
n_{x} x=x & n_{\xi} x=0 \\
n_{x} \xi=0 & n_{\xi} \xi=\xi
\end{array}
$$

and assume that they obey the usual Leibniz rule, e.g.

$$
n_{i} F:=\left(n_{i} F\right)+F n_{i} .
$$

Then on a monomial

$$
\begin{aligned}
& n_{x} \xi^{i} x^{m}=m \xi^{i} x^{m} \\
& n_{\xi} \xi^{i} x^{m}=i \xi^{i} x^{m}
\end{aligned} \quad(i=0,1) .
$$

Scaling operators can be constructed by exponentiating the number operators. For example

$$
\begin{aligned}
& q^{n=} \xi^{i} x^{m}=q^{m} \xi^{i} x^{m}=\xi^{i}(q x)^{m} \\
& q^{n} \xi^{i} x^{m}=q^{i} \xi^{i} x^{m}=(q \xi)^{i} x^{m}
\end{aligned}
$$

and for an arbitrary function

$$
\begin{aligned}
& q^{n_{s}} F(x, \xi)=F(q x, \xi) \\
& q^{n^{\prime}} F(x, \xi)=F(x, q \xi) .
\end{aligned}
$$

By analogy with the $x$ derivatives, it is convenient to introduce the formal inverse of $\xi$ which should only be used with the number operator for $\xi$. For consistency with the q-relations it must obey

$$
\xi^{-1} x=q x \xi^{-1} .
$$

Then on a general function

$$
\xi^{-1} n_{\xi}\left(F_{0}(x)+\xi F_{1}(x)\right)=\xi F_{1}(x)
$$

Now it is straightforward to read off the appropriate representation of the derivatives from their action on functions in $(4.18,4.20)$. For the solution $r=q$

$$
\begin{aligned}
& \partial_{x}=x^{-1} q^{2 n_{\ell}} \frac{q^{2 n_{s}}-1}{q^{2}-1} \\
& \partial_{\xi}=\xi^{-1} n_{\xi}
\end{aligned}
$$


and for $r=q^{-1}$

$$
\begin{aligned}
& \partial_{x}=x^{-1} \frac{q^{-2 n_{x}}-1}{q^{-2}-1} \\
& \partial_{\xi}=\xi^{-1} n_{\xi} q^{-2 n_{x}} .
\end{aligned}
$$

Note that in the limit $q \rightarrow 1$ both solutions approach the usual derivatives.

Before writing the coproduct form of the operator equations, we first note that since a monomial can have only zero or one power of $\xi$, the number operator $n_{\xi}$ can be written for arbitrary $s$ as

$$
n_{\xi}=\frac{q^{s m_{\xi}}-1}{q^{s}-1}
$$

For the solution $r=q$ this allows us to rewrite the term in (4.19) as

$$
1+\left(q^{2}-1\right) \xi \partial_{\xi}=q^{2 n_{\xi}}
$$

Also, for $r=q^{-1}$, in (4.21)

$$
1+\left(q^{-2}-1\right) x \partial_{x}=q^{-2 n s} .
$$

These relations allow a simpler form for the operator equations.

As mentioned earlier, the operator equations $(4.19,4.21)$ tell how the derivatives act on a pair of functions. If we regard each of the functions as lying in a vector space, then the operator equations are similar to the coproduct discussed for the algebra. We can read the coproduct from $(4.19,4.21)$, using the above relations for simplification. For the solution $r=q$ we find

$$
\begin{aligned}
& \Delta\left(\partial_{x}\right)=\partial_{x} \otimes q^{2 n_{\xi}}+q^{2 n_{x}+n_{\xi}} \otimes \partial_{x} \\
& \Delta\left(\partial_{\xi}\right)=\partial_{\xi} \otimes 1+q^{n_{s}} \otimes \partial_{\xi}
\end{aligned}
$$

and for $r=q^{-1}$

$$
\begin{aligned}
& \Delta\left(\partial_{x}\right)=\partial_{x} \otimes 1+q^{-2 n_{x}-n_{\xi}} \otimes \partial_{x} \\
& \Delta\left(\partial_{\xi}\right)=\partial_{\xi} \otimes q^{-2 n_{x}}+q^{-n_{z}} \otimes \partial_{\xi} .
\end{aligned}
$$

These coproducts are consistent with the derivative algebra (4.7). 


\subsection{Representation of the Algebra}

The quantum super-plane coordinates were introduced as a vector acted on by the quantum group $\mathrm{GL}_{\mathrm{q}}(1 \mid 1)$. This description also leads to the action of the quantum algebra on the super-plane. Using the number operators, we will be able to write a representation of the generators similar to the representations of the derivatives.

Begin by writing the coordinate vector $V$ as a variable quantum matrix $A$ acting on a constant vector $V_{0}$ :

$$
V=A V_{0}
$$

Now apply the exterior derivative to $V$. Since $V_{0}$ is constant, $\delta V_{0}=0$ and we have

$$
\delta V=\Omega A V_{0}=\Omega V
$$

where we have used $\delta A=\Omega A$ from (3.3). This relation is invariant under a rotation of $V_{0}$ by a constant matrix $A_{1}$ :

$$
\begin{aligned}
& V=A_{0}^{\prime} V_{0}^{\prime}=\left(A_{0} A_{1}^{-1}\right)\left(A_{1} V_{0}\right) \\
& \delta V=\Omega^{\prime} V=\Omega V
\end{aligned}
$$

where we have used the fact that the Cartan-Maurer forms are invariant under right-multiplication by a constant matrix. Thus this description is independent of the particular choice of $V_{0}$.

The matrix equation (4.27) allows us to read off the action of $\delta$ on the coordinates. We find

$$
\begin{aligned}
& \delta x=\left(\omega_{X}+(1-\phi) \omega_{Y}\right) x+v_{+} \xi \\
& \delta \xi=\left(\omega_{X}-(1+\phi) \omega_{Y}\right) \xi+v_{-} x
\end{aligned}
$$

where $\phi$ is the arbitrary mixing parameter introduced in (3.11). Using the expansion of $\delta$ in (3.6), the action of the generators is seen to be

$$
\begin{array}{rlrl}
X_{x}=x & X \xi & =\xi \\
Y_{x} & =(1-\phi) x & Y \xi & =-(1+\phi) \xi \\
\nabla_{+} x & =\xi & \nabla_{+} \xi & =0 \\
\nabla_{-} x & =0 & \nabla_{-} \xi & =x .
\end{array}
$$


Note that the generator $X$ acts much like the identity. This will not be true on an arbitrary monomial.

Next we wish to obtain the action of the generators on products of coordinates. This can be expressed in the form of operator equations similar to those obtained for the derivatives. Again we start with the exterior derivative and its Leibniz rule. Applying it to a product of coodinates, we can then commute the Cartan-Maurer forms to the left and read off the action of the generators. (This follows exactly the procedure used for the algebra in chapter 3.) So we must know how to commute Cartan-Maurer forms through coordinates. From (4.26) we see that the coordinates may be written

$$
\begin{aligned}
& x=a x_{0}+\beta \xi_{0} \\
& \xi=\gamma x_{0}+d \xi_{0} .
\end{aligned}
$$

Here the group parameters correspond to the matrix $A$ used to define the CartanMaurer forms by $\Omega=\delta A A^{-1}$. We know how to commute the 1 -forms through these group parameters from chapter 3 . On the other hand, the constant coordinates $x_{0}$ and $\xi_{0}$ are not related to $A$, so the 1-forms will obey the classical (graded) commutation rules with them. So we see that the Cartan-Maurer forms commute with the coordinates the same as with group parameters. These relations are the same for all group parameters, and are given in (3.10,3.12). Then for the coordinates we write

$$
\begin{array}{ll}
x v_{ \pm}=q v_{ \pm} x & \xi v_{ \pm}=q v_{ \pm} \xi \\
x \omega_{X}=f \omega_{X} x & \xi \omega_{X}=-f \omega_{X} \xi \\
x \omega_{Y}=\omega_{Y} x & \xi \omega_{Y}=-\omega_{Y} \xi .
\end{array}
$$

Here we consider both solutions, $f=q$ or $q^{2}$.

Now applying $\delta$ to a coordinate, using the Leibniz rule, and commuting the 1 -forms to the left we find the operator equation for the generators acting on 
coordinates. Again using $(\cdot)$ to indicate arbitrary functions to the right, we find

$$
\begin{array}{rlrl}
X x & =x \cdot+f x X . & X \xi \cdot=\xi \cdot+f \xi X \\
Y_{x}=(1-\phi) x \cdot+x Y . & Y \xi \cdot=-(1+\phi) \xi \cdot+\xi Y \\
\nabla_{+} x=\xi \cdot+q x \nabla_{+} \cdot & \nabla_{+} \xi \cdot=-q \xi \nabla_{+} \\
\nabla_{-} x \cdot=q x \nabla_{-} & \nabla_{-} \xi \cdot=x \cdot-q \xi \nabla_{-}
\end{array}
$$

Note that $Y$ does not have any factors of $q$ in its equation. It obeys an operator equation similar to the classical case, and we will not consider it further.

Finally, we may iterate the above equations to determine the operator relations for the generators on an arbitrary monomial in $x$ and $\xi$. Then any function can be written as a sum of monomials. Writing an arbitrary function $3 s$ $F(x, \xi)=F_{0}(x)+\xi F_{1}(x)$ like before, we find

$$
\begin{aligned}
& X F(x, \xi) \cdot=\frac{F(f x, f \xi)-F(x, \xi)}{f-1}+F(f x, f \xi) X \\
& \nabla_{+} F(x, \xi) \cdot=\xi x^{-1} \frac{F\left(q^{2} x, \xi\right)-F(x, \xi)}{q^{2}-1}+(-1)^{F} F(q x, q \xi) \nabla_{+} \\
& \nabla_{-} F(x, \xi) \cdot=x F_{1}(x) \cdot+(-1)^{F} F(q x, q \xi) \nabla_{-} \cdot
\end{aligned}
$$

As we did for the derivatives, taking the above equations with no function to the right of $F$ gives the action of the generators on $F$ alone. Then we can use the number operators to write a representation of the quantum generators. The result is

$$
\begin{aligned}
X & =\frac{f^{n_{s}+n_{\xi}}-1}{f-1} \\
\nabla_{-} & =\xi x^{-1} \frac{q^{2 n_{s}}-1}{q^{2}-1} \\
\nabla_{+} & =x \xi^{-1} n_{\xi}
\end{aligned}
$$

where $f=q$ or $q^{2}$. Note the similar form to the derivative representation in equations $(4.22,4.23)$. Finally, we could use the operator equations to determine the coproduct. This would give the same result as before. This is because both derivations use the same q-relations between 1-forms and coordinates or group parameters. In fact, we could have started with the previously determined coproduct and applied it to monomials to determine the action of the generators. 


\section{Chapter 5}

\section{Quotient Space Geometry}

In this chapter we develop the geometry of a quotient space. Cartan-Maurer forms are constructed in the usual way. Although they are not invariant, invariant bilinear forms can be constructed on the manifold. A symmetric bilinear provides a Riemannian metric, for which we discuss the Riemannian geometry. An invariant almost complex structure is constructed, and extended to a complex structure. If the complex manifold admits a closed hermitian 2-form, it is Kähler. In this case we find a simple form for the Ricci curvature. Details of the mathematics may be found in [19] volume 2.

\subsection{Quotient Space $G / H$}

We begin by reviewing the Riemannian geometry of a quotient space [20]. Consider a real group $G$ with subgroup $H$. For a fixed $m \in G$, the set of $g \in G$ of the form $g=m h$ as $h \in H$ runs through all of $H$ defines the left coset $g=m H$ represented by $m$. The space of all such cosets is the quotient space $\mathcal{M}=G / H . G$ may be regarded as a fiber bundle over $G / H$ with fibers $H$. Decompose the algebra of $G$ by $G=M \oplus H, M \cap H=\emptyset$. We will use indices $r, s, t, \ldots$ for generators $L_{\tau} \in G, a, b, c, \ldots$ for $L_{a} \in M$, and $i, j, \ldots$ for $L_{i} \in H$. The generators of $G$ obey $\left[L_{r}, L_{s}\right]=L_{t} C^{t}{ }_{r s}$, and for a closed subalgebra $H \subset G, C^{a}{ }_{i j}=0$. Define the exponentials $g\left(y^{r}\right)=\exp \left(L_{r} y^{r}\right), m\left(x^{a}\right)=\exp \left(L_{a} x^{a}\right)$, and $h\left(t^{i}\right)=\exp \left(L_{i} t^{i}\right)$. 
Then any element $g\left(y^{r}\right)$ may be uniquely written as

$$
g\left(y^{r}\right)=m\left(x^{a}\right) h\left(t^{i}\right)
$$

The parameters $x^{a}$ are coordinates for the element of $\mathcal{M}$ represented by $m\left(x^{a}\right)$, which defines a local section of the bundle by $m(x): G / H \rightarrow G$. The left action of $G$ on $\mathcal{M}$ is defined by left multiplication of $m\left(x^{a}\right)$ by an element of $G$ and refactoring the product as in (5.1):

$$
\begin{aligned}
& g_{0} m\left(x^{a}\right)=g\left(y^{\tau}\right)=m\left(x^{a}\right) h\left(t^{i}\right) \\
& g_{0}: m\left(x^{a}\right) \mapsto m\left(x^{\prime a}\right)=g_{0} m\left(x^{a}\right) h^{-1}\left(t^{i}\right) .
\end{aligned}
$$

The Cartan-Maurer 1-forms on $\mathcal{M}$ are defined by

$$
m^{-1} d m=L_{r} e^{r} .
$$

In components $e^{r}(x)=e_{a}^{r}(x) d x^{a}$, where the upper index $(r)$ is a label taking values in $G$, and the lower index $(a)$ is a coordinate index taking values in $M$. The forms obey the Cartan-Maurer equations

$$
d e^{r}=-\frac{1}{2} C^{r} s e^{s} e^{t} .
$$

The $e^{a}\left(L_{a} \in M\right)$ at each point $x \in \mathcal{M}$ span the cotangent space $T \mathcal{M}_{x}^{*}$. For these forms define inverse vector fields $E_{a}(x)=E^{b}{ }_{a}(x) \partial_{b}$ which span the tangent space $T M_{x}$ and obey

$$
e^{a}\left(E_{b}\right)=e^{a}{ }_{c} E_{b}^{c}=\delta_{b}^{a} .
$$

Under the left G-action defined in (5.2), the Cartan-Maurer forms transform as

$$
\begin{aligned}
\left(m^{\prime}\right)^{-1} d m^{\prime} & =h\left(m^{-1} d m\right) h^{-1}+h d h^{-1} \\
L_{r} e^{r} & =h L_{s} h^{-1} e^{s}+h d h^{-1} .
\end{aligned}
$$

The forms transform by an adjoint $H$ rotation. The transformed $e^{i}$ associated with generators in $H$ also have an inhomogeneous piece from the last term in (5.6). For the $e^{a}$ associated with generators in $M$, the infinitessimal form of (5.6) is $e^{\prime a}=e^{a}+t^{i} \delta_{L}, e^{a}$ where

$$
\begin{aligned}
& \delta_{L_{i}} e^{a}=-C^{a}{ }_{i b} e^{b} \\
& \delta_{L_{1}} E_{a}=E_{b} C^{b}{ }_{i a}
\end{aligned}
$$


where the second equation is obtained using (5.5).

Although the Cartan-Maurer forms are not left $G$-invariant, invariant bilinear forms may be constructed. Consider

$$
\begin{aligned}
& N=N_{a b} e^{a} e^{b} \\
& N(U, V)=N_{a b} e^{a}(U) e^{b}(V) \quad U, V \in \mathrm{T} \mathcal{M} .
\end{aligned}
$$

$N$ is left $G$-invariant if the coefficients $N_{a b}$ are constant on $\mathcal{M}$ and if for $\delta_{L_{i}}$ defined in (5.7) $\delta_{L_{i}} N=0$ for all $L_{i} \in H$. This is true if

$$
\begin{aligned}
& \partial_{a} N_{b c}=0 \\
& N_{a b} C^{e}{ }_{i b}+N_{e b} C^{e}{ }_{i a}=0 \quad \text { for all } L_{i} \in H .
\end{aligned}
$$

$N$ is a 2-form if $N_{a b}=-N_{b a}$. If $N_{a b}=N_{b a}, N$ is an inner product of vectors on $\mathcal{M}$.

\subsection{Riemannian Geometry of $G / H$}

For a nondegenerate tensor $N_{a b}=N_{b a}$ obeying (5.9), we take $g_{a b} \equiv N_{c d} e^{c}{ }_{a} e^{d}$ to be the Riemannian metric on $\mathcal{M}$ with line element

$$
\begin{aligned}
d s^{2} & =g_{a b} d x^{a} d x^{b} \\
& =N_{c d} e^{c}{ }_{a} e_{b}^{d} d x^{a} d x^{b} .
\end{aligned}
$$

The Cartan-Maurer forms may be regarded as vielbein for this metric.

A covariant derivative acts on vectors $V=V^{a} E_{a}$ as

$$
\nabla V=\left(d V^{a}+\omega^{a}{ }_{b} V^{b}\right) E_{a} \text {. }
$$

The connection form $\omega^{a} b$ is uniquely determined by the requirements of vanishing torsion:

$$
T^{a}=d e^{a}+\omega_{b}^{a} e^{b}=0
$$

and metric consistency:

$$
\nabla g_{a b}=0 \Rightarrow N_{a e} \omega_{b}^{e}+N_{b e} \omega_{a}^{e}=0
$$


For convenience define the constants

$$
D^{a}{ }_{b c}=N^{a e} C^{f}{ }_{b e} N_{f o} .
$$

Then the connection $\omega^{a}{ }_{b}=\omega^{a}{ }_{r b} e^{r}$ has components

$$
\begin{aligned}
& \omega^{a}{ }_{b b}=\frac{1}{2}\left(C^{a}{ }_{b b}-D^{a}{ }_{b b}-D^{a}{ }_{b c}\right) \\
& \omega^{a}{ }_{i b}=C^{a}{ }_{i b} .
\end{aligned}
$$

Finally, the curvature 2 -form is defined by $\nabla \nabla V=R^{a}{ }_{b} V^{b} E_{a}$ with

$$
\begin{aligned}
& R_{b}{ }_{b}=d \omega_{b}{ }_{b}+\omega^{a}{ }_{c} \omega^{c}{ }_{b} \\
& =\frac{1}{2} R_{c d}{ }_{b} e^{c} e^{d} \text {. }
\end{aligned}
$$

The form of the Riemann tensor $R_{c d}{ }^{a}{ }_{b}$ for $\omega^{a}{ }_{b}$ in $(5.15)$ is complicated and we will not write it. However, as noted in the second line of (5.16) all dependence on the inhomogeneously transforming $e^{i}$ vanishes.

\subsection{Almost Complex. Structure on $G / H$}

An almost complex structure on a real manifold $\mathcal{M}$ is a tensor field $J$ which at each point $x \in \mathcal{M}$ is an automorphism of the tangent space $T \mathcal{M}_{x}$ obeying $J^{2}=-1$ :

$$
\begin{gathered}
J: V \mapsto J(V) \text { for } V \in T \mathcal{M} \\
J^{2}(V)=-V .
\end{gathered}
$$

Now consider the complexified tangent space $T \mathcal{M}_{\mathbf{c}}=\mathrm{TM} \otimes \mathrm{C}$. Construct the projection operators

$$
P=\frac{1}{2}(1-i J) \quad \bar{P}=\frac{1}{2}(1+i J)
$$

which obey

$$
J P=i P \quad J \bar{P}=-i \bar{P} .
$$

Thus $P$ and $\bar{P}$ project onto subspaces of TMc with eigenvalues under $J$ of $+i$ and $-i$, respectively. Split $T \mathcal{M}_{\mathbf{c}}$ into subspaces $T \mathcal{M}_{\boldsymbol{c}}=\mathrm{T} \mathcal{M}^{(1,0)} \oplus \mathrm{T} \mathcal{M}^{(0,1)}$ by 
the mappings $P: \mathrm{T} \mathcal{M} \rightarrow \mathrm{T} \mathcal{M}^{(1,0)}$ and $\bar{P}: \mathrm{TM} \rightarrow \mathrm{T} \mathcal{M}^{(0,1)}$. The complex vectors obey

$$
\begin{array}{ll}
J V^{(1,0)}=i V^{(1,0)} & V^{(1,0)} \in \mathrm{T} \mathcal{M}^{(1,0)} \\
J V^{(0,1)}=i V^{(0,1)} & V^{(0,1)} \in \mathrm{T} \mathcal{M}^{(0,1)} .
\end{array}
$$

For components, the real indices $a, b, c, \ldots$ will be replaced by the complex indices $l, m, n, \ldots$ for $(1,0)$ vectors and $\bar{l}, \bar{m}, \bar{n}, \ldots$ for $(0,1)$ vectors. For example, a vector $V=V^{a} \partial_{a}$ is complexified and split into $V^{(1,0)}=V^{m} \partial_{m}$ and $V^{(0,1)}=V^{m} \partial_{m}$, with $\overline{V^{(1,0)}}=V^{(0,1)}$. All of these constructions with $J$ on the tangent space $\mathrm{T} M$ carry over naturally to the cotangent space $\mathrm{T} \mathcal{M}^{*}$. Also, we may choose a complex basis for the antihermitian generators $L_{a}$ such that $L_{m}^{\dagger}=-L_{m}$. This implies $\overline{e^{m}}=e^{m}$ and $\overline{E_{m}}=E_{m}$.

For a quotient space $\mathcal{M}=G / H, J$ can be expanded in the vectors and forms constructed on $\mathcal{M}$ as

$$
\begin{aligned}
& J=E_{a} J^{a}{ }_{b} e^{b} \\
& J^{2}=-1 \Rightarrow J^{a}{ }_{c} J_{b}^{c}=-\delta_{b}^{a} .
\end{aligned}
$$

In complex coordinates, (5.19) shows that

$$
J_{n}^{m}=i \delta_{n}^{m} \quad J_{n}^{m}=-i \delta^{m}{ }_{n} .
$$

$J$ will be left $G$-invariant if the tensor $J^{a}{ }_{b}$ obeys

$$
\begin{aligned}
& \partial_{a} J^{b}{ }_{0}=0 \\
& C^{a}{ }_{i c} J^{c}{ }_{b}-J^{a}{ }_{c} C^{c}{ }_{i b}=0
\end{aligned}
$$

or in complex components

$$
C^{m}{ }_{\text {in }}=0 \quad C^{m}{ }_{\text {in }}=0 .
$$

$J$ is invariant if generators in $H$ do not mix barred and unbarred generators.

\subsection{Complex Structure on $G / H$}

As described so far, the almost complex structure $J$ is a way of splitting the real valued tangent vectors into conjugate pairs of complex yalued vectors. $J$ is said 
to be integrable if it can be derived from holomorphic coordinates on a complex manifold. (On such a manifold, the transition functions between different coordinate patches are holomorphic, or complex analytic, functions of the coordinates. The manifold is said to have a complex structure.) There are several equivalent conditions for integrability of $J[19]$. Recall that $J$ was used to define the $(1,0)$ and $(0,1)$ spaces of vectors and forms with unbarred and barred components, repectively. Generalizing, define $\mathcal{A}^{(p, q)}$ to be the space of $p+q$ forms with $p(1,0)$ indices and $q(0,1)$ indices. With this separation, $J$ is integrable if

$$
\begin{array}{lll}
d \alpha \in \mathcal{A}^{(2,0)} \oplus \mathcal{A}^{(1,1)} & \text { for } & \alpha \in \mathcal{A}^{(1,0)} \\
d \alpha \in \mathcal{A}^{(0,2)} \oplus \mathcal{A}^{(1,1)} & \text { for } & \alpha \in \mathcal{A}^{(0,1)} .
\end{array}
$$

Using the Cartan-Maurer equations (5.4) this is equivalent to the the conditions on the structure constants of $G$

$$
C_{m n}^{l}=0 \quad C_{m n}^{l}=0 .
$$

Then a quotient space $\mathcal{M}=G / H$ with structure constants for $G$ satisfying (5.24) and $(5.26)$ is a complex manifold.

\subsection{Kählerian Geometry of $G / H$}

A bilinear form is said to be hermitian if

$$
N(J U, J V)=N(U, V)
$$

Hermitian inner products $N$ (obeying $N(U, V)=N(V, U)$ ) and 2-forms $\mathcal{K}$ (obeying $\mathcal{K}(U, V)=-\mathcal{K}(V, U))$ can be related by

$$
N(U, V)=\mathcal{K}(U, J V)
$$

A nondegenerate hermitian closed 2-form $\mathcal{K}(d \mathcal{K}=0)$ on a complex manifold is called a Kähler form, and the manifold is a Kähler manifold [19] . Equation (5.28) defines the Kähler metric $d s^{2}=N(d x, d x)$. 
For complex $\mathcal{M}=G / H$ expand an invariant hermitian 2-form $\mathcal{K}$ (obeying (5.9)) as

$$
\begin{aligned}
\mathcal{K} & =\frac{1}{2} K_{a b} e^{a} e^{b} \\
& =K_{\min } e^{m} e^{\pi}
\end{aligned}
$$

where in the second line $K_{m n}=0=K_{m n}$ by (5.27). Using the Cartan-Maurer equations (5.4), $d \mathcal{K}=0$ if

$$
K_{a e} C_{b c}^{e}+K_{b e} C_{c a}^{e}+K_{\infty e} C_{a b}^{e}=0 .
$$

The Kähler metric is

$$
\begin{aligned}
& N_{a b}=K_{a c} J_{b}^{c} \\
& N_{m n}=-i K_{m n} \quad N_{m n}=i K_{m n}
\end{aligned}
$$

and (5.30) relates the components $N_{m n}=N_{n m}$. In terms of the constants defined in (5.14) we find

$$
\begin{aligned}
& C^{m}{ }_{l n}+D^{m}{ }_{l n}-D^{m}{ }_{n l}=0 \\
& C^{m} l_{n}+D^{m} l_{n}+D^{m}{ }_{n l}=0 .
\end{aligned}
$$

Since the structure constants for a complex quotient space obey (5.24) and (5.26), the connection components (5.15) take the simple form

$$
\begin{array}{lll}
\omega_{m l}^{k}=-D^{k}{ }_{m l} & \omega^{k}{ }_{m l}=C_{m l}^{k} & \omega^{k}{ }_{i l}=C^{k}{ }_{i l} \\
\omega_{m l}^{k}=-D_{m l}^{k} & \omega_{m l}^{k}=C_{m l}^{k} & \omega^{k}{ }_{i l}=C^{k}{ }_{i l} \\
\omega^{k}{ }_{r l}=0 & \omega^{k}{ }_{r l}=0 . &
\end{array}
$$

Note that the connection matrix does not mix $(1,0)$ and $(0,1)$ components. In what follows we will consider only the $(1,0)$ subspace, with $(0,1)$ components given by complex conjugation. Also, we note that these components are equal to the components of the difference operator $\varphi=\mathcal{L}-\nabla$ discussed in previous work $[10,11]$

We now proceed to the curvature. From the definition (5.16), we find

$$
\begin{aligned}
& R_{m n}{ }^{k} l=-C_{m k^{k}}^{k} C^{k^{\prime}}{ }_{n l}-C^{k}{ }_{m i} C^{i}{ }_{n l} \\
& -D_{k^{\prime} m} C^{k^{\prime}}{ }_{n b}+C_{n k^{\prime}}^{k^{\prime}} D^{k^{\prime}}{ }_{l m}+C_{m i n}^{k^{\prime}} D_{l k^{\prime}}^{k} \text {. }
\end{aligned}
$$


Note that the Riemann curvature is a $(1,1)$ form. In fact, given a connection preserving a hermitian metric, if the coordinates are holomorphic then the curvature is purely a $(1,1)$ form [21]. This is equivalent to the conditions (5.24) and (5.26) for a complex structure.

For an $\mathrm{N}$ dimensional Kähler manifold the totally antisymmetric product

$$
\begin{aligned}
\operatorname{det}(e)=\bigwedge_{m} e^{m} & =e^{1} \wedge e^{2} \wedge \ldots \wedge e^{N} \\
& =\operatorname{det}\left(e^{m} \ell\right) d z^{1} d z^{2} \ldots d z^{N}
\end{aligned}
$$

spans the 1 complex dimensional space of $(\mathrm{N}, 0)$ forms. This is known as the canonical or determinant line bundle of the manifold. The covariant derivative and curvature of this form are

$$
\begin{aligned}
\nabla \operatorname{det}(e) & =\omega_{m}^{m} \operatorname{det}(e) \\
\nabla \nabla \operatorname{det}(e) & =R_{m}^{m} \operatorname{det}(e) .
\end{aligned}
$$

The 2-form $R$ is the Ricci curvature of the manifold and is the trace of the Riemann curvature. Taking the trace of (5.34) and using (5.32) we have

$$
\begin{aligned}
R_{m n} & =R_{m n}{ }^{l} l \\
& =-C_{m k}^{l}{ }_{m k} C_{n l}-C_{m i}^{l} C_{n l}^{i}-C^{k}{ }_{m n}\left(C^{l}{ }_{k l}+C^{\top}{ }_{k l}\right) .
\end{aligned}
$$

It can be shown that this Ricci form is closed and $G$-invariant. Note that all dependence on the metric $N_{a b}=K_{a c} J_{b}^{c}$ has vanished from the Ricci cuvature. This type of metric gives a unique result for the curvature.

\subsection{Discussion}

It is clear how to apply these results to a group $G$ with subgroup $H$. Decomposing the algebra as $G=M \oplus H, M \cap H=\emptyset$, choose an almost complex structure $J$ on $M$. The choice of $J$ may be based on the generators in $M$, e.g. $L_{m}^{\dagger}=-L_{m}$. For the $(1,0)$ and $(0,1)$ decomposition based on $J$, the structure constants of $G$ must obey (5.24) for $G$-invariance of $J$ and (5.26) for $\mathcal{M}=G / H$ to be complex. A 2 -form $\mathcal{K}$ is $G$-invariant if it obeys (5.9) and closed if it obeys (5.30). If $\mathcal{K}$ is 
hermitian, $\mathcal{M}$ is Kähler and equations (5.33-37) apply yielding the connection and curvatures.

These results are easily extended to $Z_{2}$ graded super-algebras. We have chosen index ordering so that most of the results presented need no extra grading signs. The main result, the Ricci tensor, does need to be modified. The correct grading for the trace of the Riemann tensor is

$$
R_{A B}=(-1)^{C} R_{A B}{ }^{C}{ }_{C} .
$$

Note aloo that the purely fermionic components of symmetric tensors become antisymmetric, and vice versa. See the appendix for details.

For finite dimensional groups, the results are directly valid. If $G$ is infinite dimensional, contraction of indices may involve infinite sums. These sums must converge for the results to be valid. For the examples from string theory, we will see that the trace of the sonnection diverges but the trace of the Riemann tensor converges. 


\section{Chapter 6}

\section{String Diffeomorphism Groups}

In this chapter we apply the results of the previous chapier to the diffeomorphism groups of string theory. The main goal is to calculate the Ricci curvature of quotient spaces formed from these groups. As discussed in the introduction, this corresponds to the ghost contribution to the anomaly of the energy-momentum tensor. For the bosonic string, the group is $\operatorname{Diff}\left(\mathrm{S}^{1}\right)$. There are two choices of subgroup which admit a Kähler form. For the superstring the group is super$\operatorname{Diff}\left(\mathrm{S}^{1}\right)$. In the Neveu-Schwarz sector their are two choices of subgroup which are Kähler. In the Ramond sector their is only one Kähler quotient space. Here we will calculate the Ricci curvature of these Kähler manifolds. Implications for string theory will be discussed in the next chapter.

\subsection{Quotient Spaces for the String}

First we apply the results of the last chapter to the symmetry groups of string theory. As discussed in [10], the open bosonic string in $D$ spacetime dimensions has as its phase space the space of loops in $R^{D}$. The reparameterizations of the circle $S^{1}$ form the group $G=\operatorname{Diff}\left(\mathrm{S}^{1}\right)$. The algebra $G=\operatorname{Diff}\left(\mathrm{S}^{1}\right)$ is based on the relation

$$
\left[f(\sigma) \frac{\partial}{\partial \sigma}, g(\sigma) \frac{\partial}{\partial \sigma}\right]=\left(f g^{\prime}-g f^{\prime}\right) \frac{\partial}{\partial \sigma}
$$


Fourier expanding gives the generators $L_{r}=e^{-i r \sigma} \frac{\partial}{\partial \sigma}$ which obey

$$
\begin{aligned}
& {\left[L_{r}, L_{s}\right]=i(r-s) L_{r+s} \quad r, s \in \mathbf{Z}} \\
& L_{r}^{\dagger}=-L_{-r}
\end{aligned}
$$

so the structure constants of $\operatorname{Diff}\left(\mathrm{S}^{1}\right)$ are

$$
C^{t}{ }_{r s}=i(r-s) \delta^{t}{ }_{r+s} \quad r, s, t \in \mathbf{Z} .
$$

The subalgebras of $G=\operatorname{Diff}\left(\mathrm{S}^{1}\right)$ are $H_{0}$ generated by $\left\{L_{0}\right\}$ and $H_{n}$ generated by $\left\{L_{0}, L_{+n}, L_{-n}\right\}$. The subgroups are $H_{0}=\mathrm{U}(1) \cong \mathrm{S}^{1}$ and $H_{n}=\mathrm{SL}(2, \mathrm{R})$.

Consider first Diff $\left(\mathrm{S}^{1}\right) / \mathrm{S}^{1}$ with subalgebra $H_{0}$. The indices $r \in \mathrm{Z}$ in $(6.2,6.3)$ take values $a \neq 0$ for $M$ and $i=0$ for $H_{0}$. The choice of $J$ suggested by (6.2) is

$$
\begin{array}{ll}
J_{n}^{m}=i \delta_{n}^{m} & m, n>0 \\
J_{n}^{m}=-i \delta_{n}^{m} & \bar{m}=-m<0
\end{array}
$$

so indices take values $m>0$ and $\bar{m}=-m<0$. The structure constants (6.3) obey (5.24) ( $J$ is $G$-invariant) and (5.26) ( $J$ is integrable), so $\mathcal{M}=G / H$ is complex.

From (5.9) the 2-form $\mathcal{K}=K_{a b} e^{a} e^{b}$ is $G$-invariant if $(a+b) K_{a b}=0$. Then $\mathcal{K}$ has components

$$
K_{a b}=f(a) \delta_{a+b} \quad f(-a)=-f(a)
$$

and $\mathcal{K}$ is hermitian if $f(m)$ is real. Closure of $\mathcal{K}$ requires

$$
(a+2 b) f(a)-(b+2 a) f(b)-(a-b) f(a+b)=0
$$

which is solved by

$$
f(a)=A a^{3}+B a \quad A, B \in \mathbf{R} .
$$

Thus there is a 2-parameter class of $\operatorname{Diff}\left(\mathrm{S}^{1}\right)$-invariant Kähler forms on $\operatorname{Diff}\left(\mathrm{S}^{1}\right) / \mathrm{S}^{1}$. $\mathcal{K}$ is nondegenerate if $A=0, B \neq 0$ or if $B / A \neq-n^{2}$ for all nonzero integers $n$. Note that the term proprotional to $B$ is exact, since $d e^{0}=-i \sum_{a \neq 0} a e^{a} e^{-a}$. The term proportional to $A$ is nontrivial.

Since $M=\operatorname{Diff}\left(S^{1}\right) / S^{1}$ is complex and admits a Kähler form, the results of the previous section may apply. Care must be exercised since $\mathcal{M}$ is infinite dimensional. We will discuss this case in some detail to exhibit the convergence 
which is provided by the choice of metric. The delta function form of the structure constants gives the connection and Riemann curvature without infinite sums. From (5.33) the connection is

$$
\omega_{m}^{m}=i\left((m-2 n) \theta(1+n-m)-(2 m-n) \frac{f(n)}{f(m)} \theta(m-n)\right) \delta_{r+n}^{m}
$$

The trace $R_{m \pi}{ }^{\prime} l$ yielding the Ricci curvature is now an infinite sum which may diverge. From (5.34)

$$
\begin{aligned}
R_{m n^{i} l}=\sum_{l>0} & (-(l+m)(l-2 m) \theta(m-l+1) \\
& \left.\quad-(l+m)(2 l-m) \frac{f(m)}{f(l)} \theta(l-m)+(l+2 m)(2 l+m) \frac{f(m)}{f(l+m)}\right) .
\end{aligned}
$$

The form of the metric in (6.7) with $A \neq 0$ shows that the sum of the last two terms in (6.9) converges. Furthermore, each of these terms is logarithmically divergent. Then we may shift summation indices, and find that the sum of the last two terms vanishes. Then we are left with the final result

$$
\begin{aligned}
R_{m n} & =\sum_{l=1}^{m}(l+m)(l-2 m) \delta_{m, n} \\
& =\left(-\frac{13}{6} m^{3}+\frac{1}{6} m\right) \delta_{m, n} .
\end{aligned}
$$

The Ricci form is closed and invariant as in $(6.5,6.7)$.

Now consider the subgroups $H_{n} \cong \operatorname{SL}(2, R)$ generated by $\left\{L_{0}, L_{n}, L_{-n}\right\}[22]$. Indices take values $a \neq 0, \pm n$ for $M$ and $i=0, \pm n$ for $H$. It is straightforward to show that $G$-invariant closed 2 -forms on $\mathcal{M}=G / H_{n}$ take the form of (6.5) with

$$
f(a)=A\left(a^{3}-n^{2} a\right) \quad A \in \mathbf{R}
$$

i.e. they are of the form (6.7) with $B / A=-n^{2}$. However, for $n \neq 1$, the generators $L_{ \pm n}$ mix barred and unbarred modes. Thus $J$ is not $G$-invariant according to (5.24). For $H_{1}$ generated by $\left\{L_{0}, L_{1}, L_{-1}\right\} J$ is $G$-invariant. Also $\mathcal{M}=G / H_{1}$ is complex since (5.26) is obeyed. Then for $n=1 \mathcal{K}$ in (6.11) is Kähler and the results of chapter 5 apply. The form of the metric again ensures convergence, and for $\mathcal{M}=\operatorname{Diff}\left(S^{1}\right) / S L(2, R)$ the Ricci curvature is

$$
R_{m n}=-\frac{13}{8}\left(m^{3}-m\right) \delta_{m, n} .
$$

Again the Ricci form is $G$-invariant and closed. 


\subsection{Quotient Spaces for the Superstring}

The symmetry group of the superstring, $G=$ super- $\operatorname{Diff}\left(\mathrm{S}^{1}\right)$, consists of the reparameterizations of the commuting coordinate on $S^{1}$ and a single Grassmann coordinate in superspace. The algebra is obtained by adding to $\operatorname{Diff}\left(\mathrm{S}^{1}\right)$ a set of Grassmann-odd generators $F_{\rho}$ which obey $F_{\rho}^{\dagger}=-F_{-\rho}$. We will use Greek letters for fermionic indices, which are integer valued in the Ramond sector and halfintegers in the Neveu-Schwarz sector. The algebra is extended from (6.2) to

$$
\begin{aligned}
{\left[L_{r}, L_{s}\right] } & =i(r-s) L_{r+s} \\
{\left[L_{r}, F_{\sigma}\right] } & =i\left(\frac{1}{2} r-\sigma\right) F_{r+\sigma} \\
\left\{F_{\rho}, F_{\sigma}\right\}_{+} & =2 i L_{\rho+\sigma}
\end{aligned}
$$

with structure constants

$$
\begin{aligned}
& C_{r s}^{t}=i(r-s) \delta^{t}{ }_{r+s} \\
& C_{r \sigma}^{r}=i\left(\frac{1}{2} r-\sigma\right) \delta_{r+\sigma}^{r} \\
& C^{t}{ }_{\rho \sigma}=2 i \delta^{t}{ }_{\rho+\sigma} .
\end{aligned}
$$

The subalgebras and generators are listed below.

$$
\begin{array}{ccl}
\text { Subalgebra } & \text { Sector } & \text { Generators } \\
H_{0} & \mathrm{NS}, \mathrm{R} & L_{0} \\
H_{n} & \mathrm{NS}, \mathrm{R} & L_{0}, L_{n}, L_{-n} \\
H_{\nu} & \mathrm{NS} & L_{0}, L_{n}, L_{-n}, F_{\nu}, F_{-\nu} \\
H_{\overline{0}} & \mathrm{R} & L_{0}, F_{0}
\end{array}
$$

The subgroups are $H_{0} \cong \mathrm{U}(1) \cong \mathrm{S}^{1}, H_{\mathrm{n}} \cong \mathrm{SL}(2, \mathbf{R})$, and $H_{\nu} \cong \mathrm{OSp}(1 \mid 2)$. The group $H_{\tilde{0}}$ is sometimes referred to as $\mathrm{s}-\mathrm{S}^{1}$.

In the NS sector $G$-invariant 2 -forms on $G / H_{0}$ are

$$
\mathcal{K}=\frac{1}{2} K_{a b} e^{a} e^{b}+\frac{1}{2} K_{\alpha \beta} e^{\alpha} e^{\beta}
$$

with components

$$
\begin{array}{ll}
K_{a b}=f(a) \delta_{a+b} & f(a)=A a^{3}+B a \\
K_{\alpha \beta}=g(\alpha) \delta_{\alpha+\beta} & g(\alpha)=4 A \alpha^{2}+B
\end{array} \quad A, B \in \mathbf{R} .
$$


Again the term proportional to $B$ is exact, and $\mathcal{X}$ is nondegenerate on $G / H_{0}$ if $A=0, B \neq 0$ or if $B / A \neq-n^{2}$ for all nonzero integers $n$. For $G / H_{n}, B / A=-n^{2}$, and for $G / H_{\nu}, B / A=-4 \nu^{2}$. In the $\mathrm{R}$ sector we have $G$-invariant 2 -forms on $G / H_{\tilde{0}}$

$$
\mathcal{K}=\frac{1}{2} K_{a b} e^{a} e^{b}+\frac{1}{2} K_{\alpha \beta} e^{\alpha} e^{\beta}+\frac{1}{2} K_{\alpha \beta} e^{a} e^{\beta} .
$$

The components $K_{a b}$ and $K_{\alpha \beta}$ have the same form as in (6.15) with $B=0$, and the mixed term is

$$
K_{a \beta}=h(a) \delta_{a+\beta} \quad h(a)=\Gamma a .
$$

The term proportional to the Grassmann constant $\Gamma$ is exact, since the exterior derivative of $e^{\tilde{0}}$ is $d e^{\tilde{0}}=-3 i \sum_{a=\alpha \neq 0} e^{a} e^{-a}$.

For a complex quotient space $G / H$, the subalgebra $H$ must contain all the real generators. In the Ramond sector this means the only possible choice is $H=H_{\overline{0}}$ generated by $L_{0}$ and $F_{0}$. Again the property $F_{\rho}^{\dagger}=-F_{-\rho}$ suggests taking positive modes for the $(1,0)$ subspace and negative modes for $(0,1)$. Then the only quotient spaces with invariant $J$ have subgroups $H_{0}$ and $H_{\frac{1}{2}}$ in the NS sector and $H_{\tilde{0}}$ in the $\mathrm{R}$ sector. Again the algebra ensures that $J$ is integrable, so these quotient space are Kähler. Following the procedure for the bosonic case, we see that the form of $f(a)$ and $g(\alpha)$ in (6.15) leads to a convergent trace of the Riemann tensor for $A \neq 0$. Note that the super-trace of a matrix $R$ is $R_{a}^{a}-R_{\alpha}^{\alpha}[16,23]$. Then the Ricci curvatures are

$$
\begin{aligned}
\text { NS sector, } H=S^{1}: \quad R_{m \pi} & =\left(-\frac{5}{4} m^{3}+\frac{1}{4} m\right) \delta_{m, n} \\
R_{\mu \nu} & =\left(-5 \mu^{2}+\frac{1}{4}\right) \delta_{\mu, \nu} \\
\text { NS sector, } H=O S p(1 \mid 2): \quad R_{m n} & =\left(-\frac{5}{4} m^{3}+\frac{5}{4} m\right) \delta_{m, n} \\
R_{\mu \nu} & =\left(-5 \mu^{2}+\frac{5}{4}\right) \delta_{\mu, \nu} \\
\text { R sector, } H=s-S^{1}: \quad R_{m n} & =\left(-\frac{3}{4} m^{3}\right) \delta_{m, n} \\
R_{\mu \nu} & =\left(-5 \mu^{2}\right) \delta_{\mu, \nu} .
\end{aligned}
$$

\subsection{Graded Algebras}

The algebras for string diffeomorphisms are examples of Z-graded algebras. A graded algebra $G$ may be split into submodules $G_{r}$ of finite dimension obeying

$$
\left[\underline{G}_{r}, G_{\mathbf{r}}\right] \subset \underline{G}_{r+o} \quad r, s \in Z \text {. }
$$


(The grading includes half-integers in the NS sector.) $G_{0}$ is a closed subalgebra so we may consider the quotient space $G / G_{0}$. The relation $G_{r}^{\dagger}=G_{-r}$ suggests an almost complex structure separating positive and negative graded submodules. The form of the structure constants $C^{r}{ }_{s t} \propto \delta_{s+t}^{r}$ obeys the conditions (5.24) and (5.26) so $G / G_{0}$ is complex. If a $G$-invariant Kähler form can be constructed, the expressions for the connection (5.33) and Riemann tensor (5.34) are valid and $\omega^{a}{ }_{b} \propto \delta_{b+r}^{a} e^{r}$ and $R_{b}^{a} \propto \delta_{b+m-n}^{a} e^{m} e^{n}$. But the Ricci tensor requires an infinite trace which may be convergent for some class of Kähler metrics. If the trace converges the Ricci form is given by

$$
R_{m n}=-\left(\sum_{l=1}^{m} C_{m, l-m}^{l} C_{-m, l}^{l-m}\right) \delta_{m, n}
$$

(The last term in (5.37) never contributes for $\mathcal{M}=G / G_{0}$.) Here an index $r$ takes all values of the generators spanning $G_{\text {r }}$. The NS sector is simply an extension of the bosonic case with half-integer graded submodules. But in the $\mathrm{R}$ sector there is a bosonic and fermionic component of each integer graded submodule. 


\section{Chapter 7}

\section{Associated Vector Bundles}

In this section we extend the results for Kähler quotient spaces to more general vector buindles over these manifolds. The new vector bundies are extensions or restrictions of the tangent space of the manifold. Requiring the bundle to be holomorphic constrains the choice of vector space for the fiber. There is a natural choice of fiber for quotient spaces based on a graded algebra. These results are applied to the string and superstring. Implications for the string vacuum state are discussed.

\subsection{Holomorphic Vector Bundles}

In the preceeding sections we have considered the Riemannian and Kählerian geometry of the quotient space $\mathcal{M}=G / H$. These are special cases of vector bundles over $\mathcal{M}$. In the Riemannian case the fiber at $x$ is the tangent space $T \mathcal{M}_{x}$. For $\mathcal{M}$ Kähler the fiber is the $(1,0)$ subspace of the complexified tangent space $T \mathcal{M}_{x}^{\mathbf{C}}=\mathrm{T} \mathcal{M}_{x}^{(1,0)} \oplus \mathrm{T} \mathcal{M}_{x}^{(0,1)}$. The Kähler metric is a hermitian inner product on $T \mathcal{M}_{x}^{(1,0)}$, and the connection acts within the $(1,0)$ subspace. In the case the vector bundle is holomorphic, i.e. the fiber transition functions between coordinate patches are holomorphic functions of the coordiantes.

For graded algebras we can consider related vector bundles over $\mathcal{M}=G / G_{0}$. The fibers are spanned by subsets of vector fields $E_{\mathrm{r}}$ or the Cartan-Maurer forms 
$e^{r}$. Define the vector space $T \mathcal{H}_{x}$ spanned by $E_{i}(x)$ for generators $L_{i} \in G_{0}=H$. Decompose the total space as $V_{x} \oplus V_{x}^{\perp}=T \mathcal{M}_{x}^{c} \oplus T \mathcal{H}_{x}$ and assign indices

$$
\begin{aligned}
& E_{m}(x) \in \mathrm{TM}_{x}^{(\mathbf{1}, 0)} \quad E_{m}(x) \in \mathrm{T} \mathcal{M}_{x}^{(0,1)} \quad E_{\mathrm{i}}(x) \in \mathrm{T} \mathcal{H}_{x} \\
& E_{a}(x) \in V_{x} \quad E_{a^{\prime}}(x) \in V_{x}^{\perp}
\end{aligned}
$$

where unprimed indices $a$ are in $V$ and primed indices $a^{\prime}$ are in $V^{\perp}$. As before $G$ invariance requires adjoint $G_{0}$ invariance. Then this decomposition is $G$-invariant if modes in $V$ and $V^{\downarrow}$ are not mixed by $G_{0}$. For a graded algebra this requires that each submodule $G_{r}$ is entirely in either $V$ or $V^{\perp}$.

In the Kähler case $V_{x}=\mathrm{T} \mathcal{M}_{x}^{(1,0)}$ and $V_{x}^{\perp}=\mathrm{T} \mathcal{M}^{(0,1)} \oplus \mathrm{T} \mathcal{H}_{x}$. We will treat the general case as a finite extension or restriction of the Kähler case. A hermitian inner product on $T \mathcal{M}^{\mathrm{C}}$ is defined by the Kähler metric. If $T_{x} \subset V_{x}$ the inner product must be extended by including a nondegenerate $N_{i j}$ obeying $N_{i l} C^{l}{ }_{k j}+N_{j l} C^{l}{ }_{k i}$ (where all indices $i, j, k, l$ are in $G_{0}$.) Also set $N_{i m}=0$ and $N_{i m}=0$. Then the hermitian inner product on $V$

$$
N\left(E_{a}, \overline{E_{b}}\right)=N_{a b}
$$

is preserved by the connection

$$
\omega^{a}{ }_{b}=-D^{a}{ }_{m b} e^{m}+C^{a}{ }_{m b} e^{m}+C^{a}{ }_{i b} e^{i}
$$

where $D^{a}{ }_{m b}=N^{a \varepsilon} C^{j}{ }_{m \varepsilon} N_{f b}$. This is just an extension or restriction of the Kähler connection that operates within the space $V$ spanned by the vector fields $E_{a}$.

The curvature of the vector bundle is defined by $R_{b}^{a}=d \omega^{a}{ }_{b}+\omega^{a}{ }_{c} \omega^{c}{ }_{b}$. For a holomorphic vector bundle with connection preserving a hermitian inner product, the curvature of the connection must be a $(1,1)$ form on the base manifold. The converse is also true [21]. Thus, if we choose a bundle and connection such that the curvature is a $(1,1)$ form, the bundle is holomorphic. For the connection (7.3) the curvature is a $(1,1)$ form on $G / H_{0}$ if

$$
C^{a^{\prime}}{ }_{m b}=0 \quad C_{m b}^{a}=0 .
$$

Using the form of the structure constants for a graded algebra, this is true if we decompose

$$
\begin{array}{ll}
E_{a} \in V & a>r_{0} \\
E_{a^{\prime}} \in V^{\perp} & a^{\prime} \leq r_{0} .
\end{array}
$$


The Kähler case is $r_{0}=0$ with $V=\mathrm{TM}^{(1,0)}$. Values of $r_{0}>0$ are finite restrictions and $r_{0}<0$ are finite extentions of this case.

The Ricci form $R=R_{a}^{a}$ is the curvature of the determinant line bundle constructed as the exterior product of all forms spanning $V^{*}$, the dual space of $V$. For the graded case this is the semiinfinite form

$$
\operatorname{det}_{r_{0}}(e)=\bigwedge_{a>r_{0}} e^{a}=e^{r_{0}+1} \wedge e^{r_{0}+2} \wedge e^{r_{0}+3} \cdots
$$

including all $e^{d}$ at levels higher than $r_{0}$. The cohomology of such forms has been studied in reference [24]. Again the form of the metric can ensure convergence of the trace. For a finite change in connection $\Delta \omega^{a}{ }_{b}$ from the Kähler connection, the Ricci form is

$$
R=R_{K}+d \Delta \omega_{a}^{a}
$$

where $R_{K}$ is the Ricci form of Kähler geometry. For a graded algebra each vector $E_{a}$ in $V$ contributes the exact term $-C^{i}{ }_{m n} C^{a}{ }_{i a} e^{m} e^{n}$ to the Ricci form. Adding or subtracting these terms from the Kählerian Ricci form (5.37), the Ricci curvature for any $r_{0}$ is

$$
\begin{aligned}
R_{m n} & =-C_{m b}^{a} C_{n a}^{b^{\prime}} \quad a>r_{0}, b^{\prime} \leq r_{0} \\
& =-\sum_{a=r_{0}+1}^{r_{0}+m} C^{a}{ }_{m, a-m} C^{a-m}-m, a .
\end{aligned}
$$

For $G=\operatorname{Dif}\left(\mathrm{S}^{1}\right), H=\mathrm{S}^{1}$, the line bundle det $t_{r_{0}}(e)$ of forms $e^{a}, a>r_{0}$ has curvature

$$
R_{m n}=\left(-\frac{13}{6} m^{3}+\left(r_{0}\left(r_{0}+1\right)+\frac{1}{6}\right) m\right) \delta_{m, n}
$$

For the superstring case with $H=\varepsilon_{0}$, the determinant must include fermionic modes $e^{\alpha}, \alpha>\rho_{0}$, where $\rho_{0}=r_{0}+\frac{1}{2} \delta_{0}, \delta_{0}= \pm 1(0)$ in the NS(R) sector. The curvature in the NS sector is

$$
\begin{aligned}
& R_{m n}=\left(-\frac{3}{4} m^{3}+\left(\delta_{0}\left(r_{0}+\frac{1}{2}\right)-\frac{1}{4}\right) m\right) \delta_{m, n} \\
& R_{\mu \nu}=\left(-5 \mu^{2}+\left(\delta_{0}\left(r_{0}+\frac{1}{2}\right)-\frac{1}{4}\right)\right) \delta_{\mu, \nu}
\end{aligned}
$$

The curvature in the Ramond sector does not change from the Kähler case, since the bosonic and fermionic contributions at each integer level cancel. 


\subsection{Implications for String Theory}

It is known from the study of gauge theories that the ghosts may be identified with the Cartan-Maurer forms of the gauge group. The ghost vacuum of string theory filled to level $r_{0}+1$ is the semiinfinite product

$$
\begin{aligned}
|0\rangle_{r_{0}} & =\prod_{a>r_{0}} c^{a}=c^{r_{0}+1} c^{r 0+2} c^{r 0+3} \ldots \\
c^{a}|0\rangle_{r_{0}} & =0 \quad a>r_{0}
\end{aligned}
$$

Identifying the ghost modes with the Cartan-Maurer forms, we see that this is the semiinfinite form (7.6). The ghost contribution to the energy-momentum tensor anomaly in these vacuua has been calculated [25] and agrees with our results $(7.9,7.10)$ and with reference [12] and standard results in string theory [26].

The total string vacuum is the product of matter fields $X^{\mu}(\sigma)$ vacuua and ghost vaculum: $|0\rangle=|0\rangle_{X} \otimes|0\rangle_{r_{0}}$. The contribution of the $X^{\mu}$ vacuua to the anomaly has been calculated as the curvature of a holomorphic line bundle over (super-) $\operatorname{Diff}\left(\mathrm{S}^{1}\right) / \mathrm{S}^{1}[10,12]$. For the bosonic string in $D$ spacetime dimensions this curvature is

$$
F_{m n}=\left(\frac{D}{12}\left(m^{3}-m\right)-2 a_{0} m\right) \delta_{m, n}
$$

where $a_{0}$ is a normal ordering constant for $L_{0}$, the generator of rigid $S^{1}$ rotations. The total curvature of the vacuum bundle is the sum of the string and ghost contributions. Reparameterization invariance of the vacum requires that the curvature vanish. Rewrite (7.9) in the suggestive form

$$
R_{m n}=\left(-\frac{13}{6}\left(m^{3}-m\right)+\left(r_{0}+2\right)\left(r_{0}-1\right) m\right) \delta_{m, n} .
$$

The nontrivial terms in the curvatures fix $D=26$. The exact terms fix the normal ordering constant to

$$
a_{0}=\frac{1}{2}\left(r_{0}+2\right)\left(r_{0}-1\right) .
$$

$c_{w}=-1$ for $r_{0}=0$, the vacuum corresponding to the canonical line bundle of $\operatorname{Diff}\left(\mathrm{S}^{1}\right) / \mathrm{S}^{1}$. $a_{0}$ vanishes for $r_{0}=-2,1$. This corresponds to the vacuua which just include or exclude modes for the generators of $\operatorname{SL}(2, R)$. These are the $\operatorname{SL}(2, R)$ invariant vacuua. Note that in these cases the curvature agrees with the Ricci curvature of $D$ iffil $\left(S^{1}\right) / S L(2, n)$ from $(0.12)$. 
In the Neveu-Schwarz sector of the superstring the curvature of the matter vacuum bundle is

$$
\begin{aligned}
& F_{m n}=\left(\frac{D}{8}\left(m^{3}-m\right)-2 a_{0} m\right) \delta_{m, n} \\
& F_{\mu D}=\left(\frac{D}{2}\left(\mu^{2}-\frac{1}{4}\right)-2 a_{0}\right) \delta_{\mu, \nu} .
\end{aligned}
$$

Rewrite (7.10) in the similar form

$$
\begin{aligned}
& R_{m n}=\left(-\frac{5}{4}\left(m^{3}-m\right)+\left(\delta_{0}\left(r_{0}+\frac{1}{2}\right)-\frac{3}{2}\right) m\right) \delta_{m, n} \\
& R_{\mu \nu}=\left(-5\left(\mu^{2}-\frac{1}{4}\right)+\left(\delta_{0}\left(r_{0}+\frac{1}{2}\right)-\frac{3}{2}\right)\right) \delta_{\mu, \nu}
\end{aligned}
$$

The notrivial terms fix $D=10$. The exact terms fix

$$
a_{0}=\frac{1}{2}\left(\delta_{0}\left(r_{0}+\frac{1}{2}\right)-\frac{3}{2}\right) .
$$

$a_{0}=-\frac{1}{2}$ for $r_{0}=0$ and $\delta_{0}=+1$, which corresponds to the canonical line bundle of super-Difi( $\left(S^{1}\right) / S^{1}$. $a_{0}$ vanishes for $r_{0}=1, \delta_{0}=+1$ and for $r_{0}=-2, \delta_{0}=-1$. This corresoonds to the vacuua which just include or exclude the modes for the generators of $\operatorname{OSp}(1 \mid 2)$. Here the curvature also agrees with the Ricci curvature of super-Diff( $\left(S^{1}\right) / O S p(1 \mid 2)$ from (6.17).

Finally, in the Ramond sector the curvature of the matter vacuum bundle is

$$
\begin{aligned}
& F_{m \pi}=\left(\frac{D}{8} m^{3}-2 a_{0} m\right) \delta_{m, n} \\
& F_{\mu \nu}=\left(\frac{D}{2} \mu^{2}-2 a_{0}\right) \delta_{\mu, \nu .} .
\end{aligned}
$$

The curvature of the ghost vacuum bundle is always

$$
\begin{aligned}
& R_{m n}=\left(-\frac{5}{4} m^{3}\right) \delta_{m, n} \\
& R_{\mu \nu}=\left(-5 \mu^{2}\right) \delta_{\mu, \nu}
\end{aligned}
$$

with no dependence on the sea level. Thus $a_{0}=0$ for all vacuua in the Ramond sector. 


\section{Appendix A}

\section{Super-Tensor Index Conventions}

In this appendix we discuss $Z_{2}$ grading sign conventions for super-tensors. Superspace is based on a Grassmann algebra. A set of Grassmann generators $\xi^{i}$ obey

$$
\xi^{i} \xi^{j}=-\xi^{j} \xi^{i} \quad\left(\xi^{i}\right)^{2}=0 .
$$

For most applications in physics the number of Grassmann generators is infinite. By extending the usual properties of tensors to functions including the Grassmann generators one define tensors on superspace. Due to the sign in (1.1) signs appear in many tensor relations. Here we will derive appropriate conventions to determine the signs. Our results are similar to those of [23]. When referring to c-number quantities, we use the terms commuting, Grassmann even, and bosonic interchangeably. For quantities obeying (1.1) we use the terms anticommuting, Grassmann odd, and fermionic interchangeably.

\section{A.1 Super-Functions and Derivatives}

The $Z_{2}$ grading for vectors can be inferred from the properties of functions of Grassmann variables and their derivatives. Consider functions of two variables, $x$ and $\xi$, which are commuting and anticommuting, respectively. Since Grassmann variables are nilpotent, an expansion of such a function in powers of $\xi$ will have only two terms:

$$
F(x, \xi)=F_{0}(x)+\xi F_{1}(x)
$$


Take first a function $f$ which is overall commuting. Then the expansion is

$$
f(x, \xi)=a(x)+\xi \beta(x)=a(x)-\beta(x) \xi
$$

where the coefficient function $a(x)$ is commuting and $\beta(x)$ is anticommuting. Now consider the left and right derivatives of $f$ with respect to $x$ and $\xi$. They are

$$
\begin{array}{ll}
\overrightarrow{\partial_{x}} f(x, \xi)=a^{\prime}(x)+\xi \beta^{\prime}(x) & f(x, \xi) \overleftarrow{\partial_{x}}=a^{\prime}(x)+\xi \beta^{\prime}(x) \\
\overrightarrow{\partial_{\xi}} f(x, \xi)=\beta(x) & f(x, \xi) \overleftarrow{\partial_{\xi}}=-\beta(x)
\end{array}
$$

We see that left and right $x$ derivatives are equal, but the left and right $\xi$ derivatives have opposite sign. Next consider a Grassmann valued function $\phi(x, \xi)$ with expansion

$$
\phi(x, \xi)=\alpha(x)+\xi b(x)=\alpha(x)+b(x) \xi .
$$

Here $\alpha(x)$, is anticommuting and $b(x)$ is commuting. Then the left and right derivatives are

$$
\begin{array}{ll}
\overrightarrow{\partial_{x}} \phi(x, \xi)=\alpha^{\prime}(x)+\xi b^{\prime}(x) & \phi(x, \xi) \overleftarrow{\partial_{x}}=\alpha^{\prime}(x)+\xi b^{\prime}(x) \\
\overrightarrow{\partial_{\xi}} \phi(x, \xi)=b(x) & \phi(x, \xi) \overleftarrow{\partial_{\xi}}=b(x)
\end{array}
$$

In this case we see that left and right derivatives are equal for both $x$ and $\xi$.

\section{A.2 Super-Vectors}

These results can be used to define index grading for super-vectors with upper or lower indices. As is conventional, we will take derivatives to have lower indices. Then the derivative of a function is a vector with a lower index, e.g. $U_{A}$. Since coordinates have upper indices and are functions on the vector space, we will take coordinates to be upper index vectors, e.g. $V^{A}$. Next we recall a result of DeWitt which states that any super-vector space may be chosen to have a pure basis, that is, one in which the basis vectors are either purely commuting or purely anticommuting. Then the Grassmann parity of a vector component is determined by its index. For $Z_{2}$ grading signs, we use the common notation based on the vector index $A$ :

$$
(-1)^{A}= \begin{cases}+1 & A \text { bosonic index } \\ -1 & A \text { fermionic index }\end{cases}
$$


This notation means that in the exponent of $(-1)$, replace the index by 0 if the index is bosonic and 1 if the index is fermionic. When considering more than one vector, grading signs depending on several indices may occur. Using 0 or 1 for indices in exponents of $(-1)$, we have for example

$$
(-1)^{A B}= \begin{cases}-1 & A \text { and } B \text { both fermionic } \\ +1 & \text { otherwise }\end{cases}
$$

Finally, an object may have an intrinsic Grassmann parity in addition to any index grading. For example, the above functions $f$ and $\phi$ are bosonic and fermionic, respectively. Then the grading notation is the same as for indices, writing the name of the object instead of the index in the exponent of $(-1)$. The name is then replaced by 0 or 1 if the object is bosonic or fermionic, respectively. For example, for a function $F$ :

$$
(-1)^{F}= \begin{cases}+1 & F \text { bosonic } \\ -1 & F \text { fermionic }\end{cases}
$$

Most of the super-tensors we will consider are extensions of the purely bosonic case, and as such have even intrinsic Grassmann parity. For the rest of this discussion we will assume that tensors have even intrinsic parity unless indicated otherwise.

The results above indicate that left and right derivatives may have different signs. Thus, we will have to distinguish between vectors with left and right indices. The index location indicates how a vector transforms under a change of basis. Left index vectors are multiplied by a rotation matrix on the left, and similarly for right indices. Covariance under such rotations will determine $Z_{2}$ grading signs associated with a particular quantity.

First consider lower index vectors, which can be related to the derivative of a bosonic valued function. (The derivative of a Grassmann valued function will yield a vector with odd intrinsic parity.) For a function $f$ of coordinates $x^{A}$ define a vector $U$ by

$$
U_{A}=f \frac{\overleftarrow{\partial}}{\partial x^{A}} \quad{ }_{A} U=\frac{\vec{\partial}}{\partial x^{A}} .
$$

Then from (1.1) we see that

$$
U_{A}=(-1)^{A}{ }_{A} U \text {. }
$$

For fermionic components of lower-index vectors, the left- and right-index components have oppposite sign. 
To proceed, we will have to consider the transformation properties of vectors. Suppose we change coordinates by $x^{A} \rightarrow x^{\prime A}\left(x^{B}\right)$. Define left and right transformation matrices by the derivatives

$$
{ }_{A} L^{B}=\frac{\vec{\partial}}{\partial x^{A}} x^{B} \quad{ }^{B} R_{A}=x^{B} \frac{\overleftarrow{\partial}}{\partial x^{A}} .
$$

For example, these relate the derivatives

$$
\frac{\vec{\partial}}{\partial x^{A}}={ }_{A} L^{B} \frac{\vec{\partial}}{\partial x^{\prime B}} \quad \frac{\overleftarrow{\partial}}{\partial x^{A}}=\frac{\overleftarrow{\partial}}{\partial x^{\prime B}} R_{A} .
$$

From the above results for derivatives of functions $(1.2,1.3)$, we see that the left and right matrices are related by

$$
{ }^{A} R_{B}=(-1)^{B(A+1)}{ }_{B} L^{A} .
$$

For the rest of this discussion, different index orderings of a given tensor will be referred to by the same name. Thus, $R$ and $L$ are both the same rotation matrix $M$, and

$$
{ }^{A} M_{B}=(-1)^{B(A+1)}{ }_{B} M^{A}
$$

is the grading for different index orderings. Also, note from the definition (1.4) that the Grassmann parity of ${ }^{A} M_{B}$ is $(-1)^{A+B}$.

Now we can determine all grading conventions for vectors. For a rotation matrix $M$, we will also need the inverse rotation $N$ which obeys

$$
{ }^{A} M_{B}{ }^{B} N_{C}={ }^{A} \delta_{C} \quad{ }^{A} \delta_{C}= \begin{cases}1 & A=C \\ 0 & \text { otherwise. }\end{cases}
$$

The different index orderings for $N$ are related the same as $M$. Define left- and right- , upper- and lower-index vectors to have transformation rules

$$
\begin{array}{ll}
V^{\prime A}=V^{B}{ }_{B} M^{A} & U_{A}^{\prime}=U_{B}{ }^{B} N_{A} . \\
{ }^{A} V^{\prime}={ }^{A} M_{B}{ }^{B} V & { }_{A} U^{\prime}={ }_{A} N^{B}{ }_{B} U
\end{array}
$$

Theis using (1.5) to rearrange indices on $M$ and using the Grassmann parity of the vectors and $M$, one can relate the left- and right-index vectors. For upper-index $V$ :

$$
\begin{aligned}
{ }^{A} V^{\prime} & ={ }^{A} M_{B}{ }^{B} V \\
& =(-1)^{B(A+1)}{ }_{B} M^{A}{ }^{B} V \\
& ={ }^{B} V_{B} M^{A} .
\end{aligned}
$$


Comparing with the defined transformation properties (1.6) we see that ${ }^{A} V$ and $V^{A}$ transform the same way Thus they are equal up to a multiplicative constant. Choosing the constant to be one we may identify

$$
{ }^{A} V=V^{A} .
$$

For lower-index $U$ :

$$
\begin{aligned}
U_{A}^{\prime} & =U_{B}{ }^{B} N_{A} \\
& =(-1)^{A(B+1)} U_{B} N^{B} \\
& =(-1)^{A+B}{ }_{A} N^{B} U_{B} \\
(-1)^{A} U_{A}^{\prime} & ={ }_{A} N^{B}(-1)^{B} U_{B} .
\end{aligned}
$$

Thus we see that $(-1)^{A} U_{A}$ transforms like ${ }_{A} U$ and we identify

$$
{ }_{A} U=(-1)^{A} U_{A}
$$

Again we have set an arbitrary constant to one. This agrees with our earlier conclusion for lower-index vectors defined by derivatives.

\section{A.3 Super-Tensors}

Tensors are constructed simply as the product of vectors. As an example, consider the case of a tensor with one upper and two lower indices constructed from an upper-index vector $V$ and lower-index vectors $U$ and $W$. Then define the tensor $T$ by

$$
T^{A}{ }_{B C}=V^{A} U_{B} W_{C} .
$$

Different index orderings are obtained by permuting the defining vectors and including appropriate grading signs. For example, some permutations of $T$ are

$$
\begin{aligned}
T^{A}{ }_{B C} & =(-1)^{A B} T_{B}{ }_{C} \\
& =(-1)^{A(B+C)} T_{B C}{ }^{A} \\
& =(-1)^{B C} \tilde{T}_{C B}^{A} .
\end{aligned}
$$

Note that the tensor in the last line is different from the initial one, and is defined by $T \propto V W U$, with the vectors ordeied differently from the first case. Finally, 
indices may be changed between left and right using (1.7) for upper indices and $(1,8)$ for lower indices.

The transformation rules for tensors are easily obtained from those for the defining vectors in (1.6). For the above example, with rotation matrix $M$ and inverse $N$,

$$
\begin{aligned}
T^{\prime A}{ }_{B C} & =V^{\prime A} U_{B}^{\prime} W_{C}^{\prime} \\
& =V^{D}{ }_{D}^{A} U_{E}^{E} N_{B} W_{F}^{F} N_{C} \\
& =(-1)^{E(A+D)+F(A+B+D+E)} V^{D} U_{E} W_{F} D^{A} M^{E} N_{B} F_{C} \\
& =(-1)^{E(A+D)+F(A+B+D+E)} T^{A}{ }_{B C D} M^{A} E_{N_{B}}{ }^{F} N_{C} .
\end{aligned}
$$

In general, a tensor transforms by the product of the transformation matrices for each of its indices, with grading signs included for permuting saturated indices until they are adjacent.

Consider now a tensor with one upper- and one lower-index defined by

$$
T^{A}{ }_{B}=V^{A} U_{B}
$$

We wish to construct a scalar $S$ by saturating the indices. Grading signs will be needed to insure that the scalar is invariant, i.e. $S^{\prime}=S$. From the defintion of the roatation matrix $M$ and inverse $N$, we see that the quantity $V^{A}{ }_{A} U$ is invariant:

$$
V^{\prime A}{ }_{A} U^{\prime}=V^{C}{ }_{C} M^{A}{ }_{A} N^{D}{ }_{D} U=V^{C}{ }_{C} \delta^{D}{ }_{D} U=V^{C}{ }_{C} U .
$$

But from (1.8) ${ }_{A} U=(-1)^{A} U_{A}$, so the scalar quantity is

$$
S=(-1)^{A} V^{A} U_{A}=(-1)^{A} T_{A}^{A} .
$$

This determines the appropriate grading for saturating indices on a tensor $T^{A}{ }_{B}$. It agrees with the well known form of the super-trace. Saturation of indices with other orderings, or left- or right-indices, is determined using the appropriate grading for permuting the indices. For example

$$
\begin{aligned}
S & =(-1)^{A} T^{A}{ }_{A}=T_{A}{ }^{A} \\
& =(-1)^{A}{ }^{A} T_{A}=(-1)^{A}{ }_{A} T^{A} \\
& ={ }^{A}{ }_{A} T=(-1)^{A}{ }_{A}{ }^{A} T
\end{aligned}
$$


are the ways to saturate all possible index arrangements of the tensor $T$ considered above. Finally, pairs of indices on higher rank tensors may be saturated to create a tensor with rank two lower. Grading signs are inserted to permute the indices until the saturated indices are adjacent. Then the above rules are used to sature the pair. For example,

$$
S_{B C}=(-1)^{A(1+B+C)} T^{A}{ }_{B C A}
$$

is the correct way to form a tensor $S_{B C}$ by saturating the first and last indices of a tensor $T_{B C D}^{A}$.

Finally, we note that tensors may be multiplied by saturating indices, forming a new tensor with the remaining indices. The grading rules are easily determined using the definition of a tensor (1.9) and the grading rules (1.10). The simplest case is saturating the last index of the first tensor with the first index of the last. For example

$$
R_{B}^{A}=S_{C}^{A} T_{B}^{C}
$$

forms a new rank-2 tensor $R$ from rank-2 tensors $S$ and $T$. When transformed, the rotation matrices for the saturated indices cancel, as in the case for the scalar quantity (1.10). Other index orderings are obtained by including grading signs to permute indices until the saturated indices are adjacent. For example,

$$
R^{A}{ }_{B}=(-1)^{A C} S_{C}{ }^{A} T^{C}{ }_{B}
$$

forms $R$ from $S$ and $T$, with $S$ now having index ordering $S_{A}{ }^{B}$.

The tensor multiplication rules are particularly useful when considering the tensor product of matrices. The tensor product of two matrices $R$ and $S$ can be written as a rank-4 tensor with index ordering given by

$$
(R \otimes S)^{A_{1}}{ }_{B_{1}}^{A_{2}}{ }_{B_{2}}=R^{A_{1}}{ }_{B_{1}} S^{A_{2}}{ }_{B_{2}} \text {. }
$$

The index subscripts indicate which vector space in the tensor product the index belongs to. Then multiplication of two such matrices will require grading signs from permuting the saturated indices until they are adjacent. As seen above, no grading signs are needed in the multiplication if the indices are already adjacent. Thus, when dealing with large matrices, it is convenient to arrange the inciices in 
the tensor product so that this is the case. For a tensor product, this means that the first and last indices should belong to the same vector space, the second and second to last indices belong to the same vector space, and so forth for higher tensor products. For the above example, this appropriate arrangement is

$$
(R \otimes S)^{A_{1} A_{2}}{ }_{B_{2} B_{1}}=(-1)^{B_{1}\left(A_{2}+B_{2}\right)} R^{A_{1}} B_{1} S^{A_{2}} B_{2} .
$$

Then two such matrices may be multiplied using normal matrix multiplication with no grading. For example

$$
(R \otimes S)^{A_{1} A_{2}}{ }_{B_{2} B_{1}}=\left(R^{\prime} \otimes S^{\prime}\right)^{A_{1} A_{2}} C_{2} C_{2}\left(R^{\prime \prime} \otimes S^{\prime \prime}\right)^{C_{1} C_{2}}{ }_{B_{2} B_{1}} .
$$

Essentially this convention moves the grading signs to the definition of the matrix tensor product. This convention is most convenient when dealing with the tensor product of two or more matrices. For example, the triple tensor product of two $2 \times 2$ matrices is an $8 \times 8$ matrix. Including the signs when writing these large matrices allows normal matrix multiplication, avoiding sign errors in the tedious manipulations. 


\section{Bibliography}

[1] V.G. Drinfeld, "Quantum Groups", Proc. Internat. Congr. Math., vol. 1, 798820, Berkeley (1986).

[2] M. Jimbo, Lett. Math. Phys. 11247 (1986).

[3] L.D. Faddeev, "Integrable Models in (1+1)-dimensional Quantum Field Theory", (Les Houches, 1982), Elsevier Science Publishers, Amsterdam, 1984.

[4] L. Alvarez-Gaumé, C. Gomez and G. Sierra, "Duality and Quantum Groups", CERN-TH.5369/89, UVGA-DPT-3/605/89, as well as earlier CERN and UVGA preprints.

[5] Yu.I. Manin, "Quantum Groups and Non-Commutative Geometry", Montréal University preprint, CRM-1561 (1988).

[6] L.D. Faddeev, N. Reshetikhin and L.A. Takhtajan, "Quantization of Lie Groups and Lie Algebras", LOMI E-14-87 (1987), to appear in M. Sato's 60th birthday volume.

[7] L.A. Takhtajan, "Quantum Groups and Integrable Models", Advanced Studies in Pure Mathematics 19, 1989.

[8] S.L. Woronowicz, "Twisted SU(2) Group. An Example of Non-Commutative Differential Calculus", Publ. RIMS-Kyoto 23117 (1987).

[9] J. Wess and B. Zumino, "A Right-Invariant Calculus for $G L_{q}(2, C)^{n}$, to appear.

[10] M.J. Bowick and S.G. Rajeev, Phys. Rev. Lett. 58, 535 (1987); Nucl. Phys. B293, 318 (1987); The Complex Geometry of String Theory and Loop Space, 
Invited talks at the Eleventh Johns Hopkins Workshop on Current Problems in Particle Theory, Lanzhou, People's Republic of China, June 17-19, 1987.

[11] B. Zumino, The Geometry of the Virasoro Group for Physicists, Lectures presented at the Cargèse 1987 Summer School on Particle Physics, Cargèse, Corsica, August 3-21, 1987. LBL preprint LBL-24319, UCB preprint UCB$\mathrm{PTH}-87 / 48$.

[12] K. Pilch and N.P. Warner, Journ. Classical and Quantum Gravity, MIT preprint, (CTP 1457, Feb. 1987).

[13] D. Harari, D.K. Hong, P. Ramond and V.G.J. Rodgers, Nucl. Phys. B294, 556 (1987).

[14] P. Oh and P. Ramond, Phys. Lett. B 195, 130 (1987).

[15] Z. Zhao, K. Wu and T. Saito, Phys. Lett. B 199, 37 (1987).

[16] W.B. Schmidke and S.P. Vokos, Ann. Phys. 189, 190 (1989).

[17] V. Jones, private communication.

[18] J. Schwenk, W.B. Schmidke and S. Vokos, "Properties of $2 \times 2$ Quantum Matrices in $\mathbf{Z}_{2}$-graded Spaces", preprint LBL-27933, UCB-PTH-89/25 (1989).

[19] S. Kobayashi and K. Nomizu, Foundations of Differential Geometry, (Interscience, New York, 1969), Vol. I, Chapter VI, Sec. 2,3. Vol. II, Chapter IV. This is a standard textbook on modern differential geometry.

[20] R. Coquereaux and A. Jadczyk, Riemannia.. Geometry, Fiber Bundles, Kaluza-Klein Theories and all that .... World Scientific (1988).

[21] S. Kobayashi, Differential Geometry of Complex Vector Bundles. Princeton University Press (1987).

[22] M.J. Bowick and A. Lahiri, J. Math. Phys. 29, 1979 (1988).

[23] B. DeWitt. Supermanifolds. Cambridge University Press (1984). 
[24] I.B. Frenkel, H. Garland and G.J. Zuckerman Proc. Natl. Acad. Sci. USA 83, 8442 (1986).

[25] D. Friedan, E. Martinec and S. Shenker, Nucl. Phys. B198, 93 (1986).

[26] M.B. Green, J.H. Schwarz and E. Witten. Superstring Theory. Cambridge University Press (1987). This textbook contains numerous references to original works. 

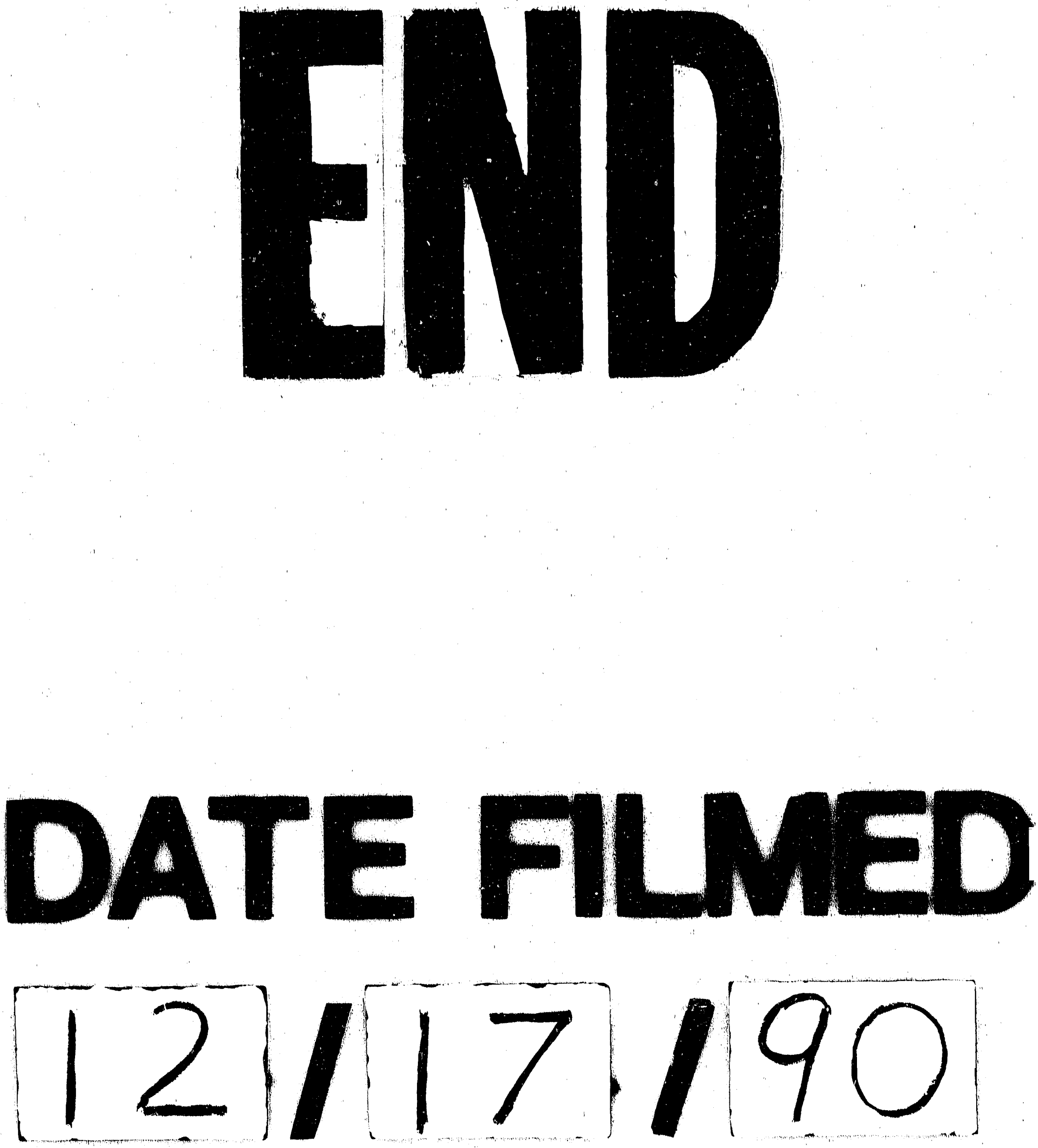
